Aus der Abteilung Neurochirurgie

(Prof. Dr. med. V. Rohde)

im Zentrum Neurologische Medizin

der Medizinischen Fakultät der Universität Göttingen

\title{
Dynamische transpedikuläre Verschraubung der Lendenwirbelsäule am Beispiel des Bricon DSS-Systems. Klinische und radiologische Ein-Jahres-Ergebnisse
}

\author{
INAUGURAL - DISSERTATION \\ zur Erlangung des Doktorgrades \\ der Medizinischen Fakultät \\ der Georg-August-Universität zu Göttingen \\ vorgelegt von \\ Sabrina Monika Greve \\ aus \\ Fulda
}

Göttingen 2010 
Dekan: Prof. Dr. med. C Frömmel

I. Berichterstatter: Prof. Dr. med. V. Rohde

II. Berichterstatter/in:

III. Berichterstatter/in:

Tag der mündlichen Prüfung: 
Inhaltsverzeichnis

1 Einleitung 1

1.1 Anatomische Aspekte und klinische Bedeutung der Instabilität der Lendenwirbelsäule 1

1.1.1 Anatomische Grundlagen.......................................................... 1

1.1.2 Stadien der Degeneration ............................................................ 2

1.1.3 Klinische Bedeutung der degenerativen Instabilität .......................... 3

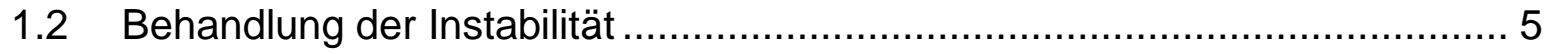

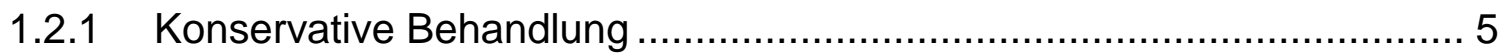

1.2.2 Die operative Behandlung der Instabilität der Wirbelsäule................... 5

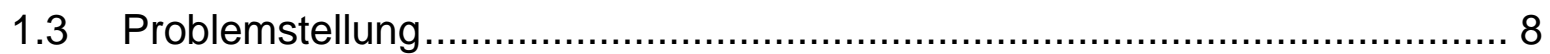

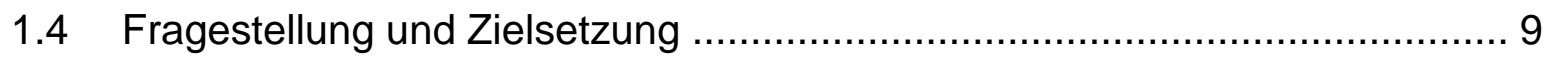

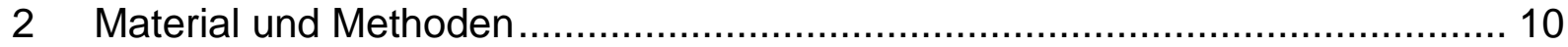

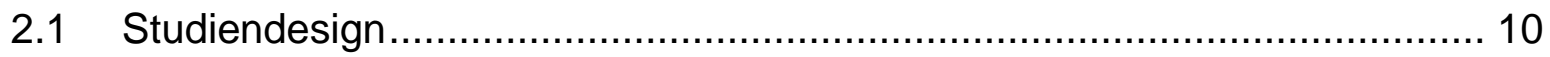

2.1.1 Patientenselektion..................................................................... 10

2.1.2 Klinische Nachuntersuchung:.................................................. 16

2.1.3 Radiologische Nachuntersuchung: ....................................... 19

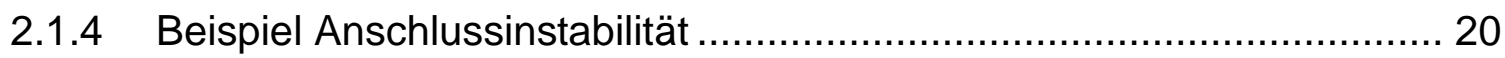

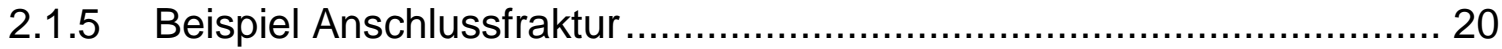

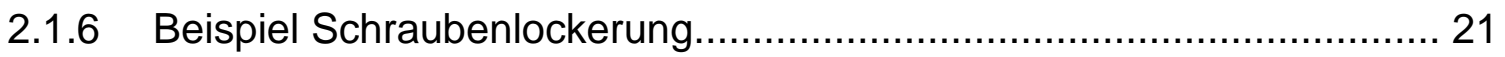

2.1.7 Beispiel Schraubenbruch ....................................................... 21

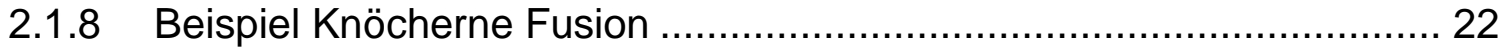

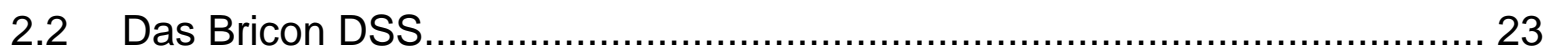




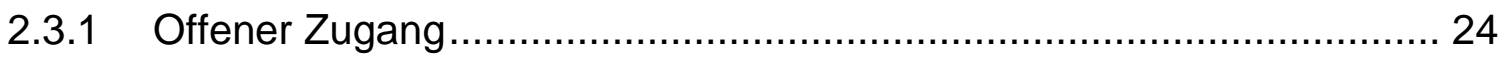

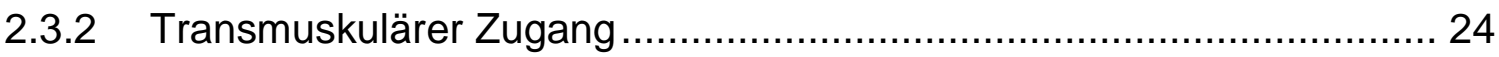

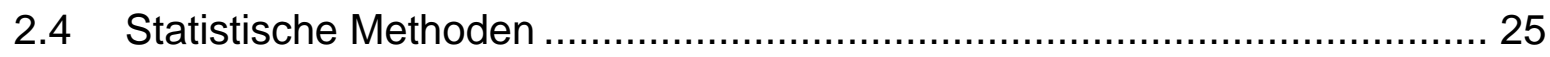

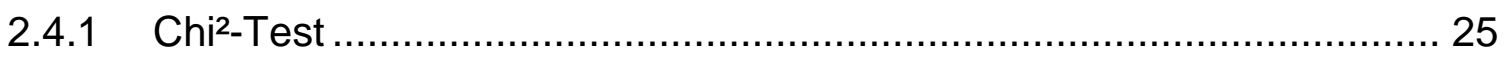

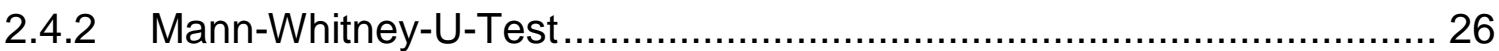

2.4.3 Logistische Regression ............................................................ 26

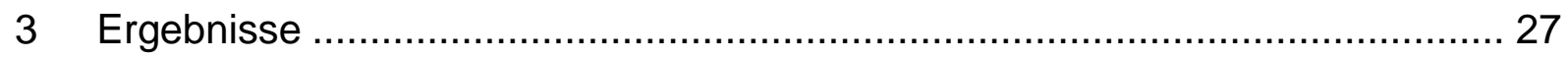

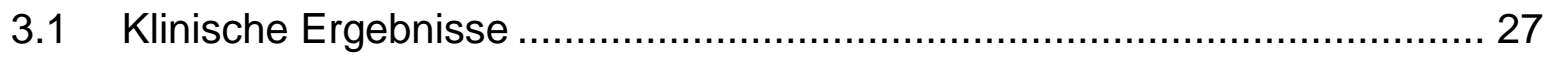

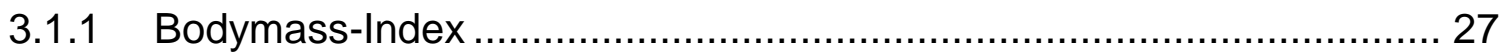

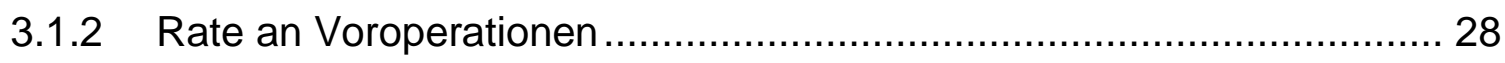

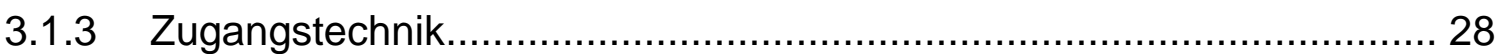

3.1.4 Entwicklung des Schmerzmittelbedarfs postoperativ .......................... 29

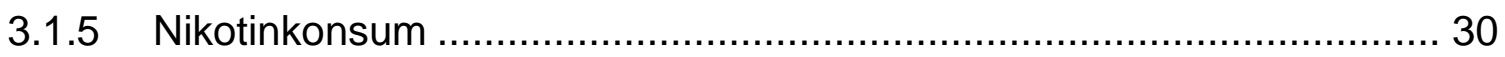

3.1.6 Auswertung des Schmerzempfindens nach VAS ............................... 31

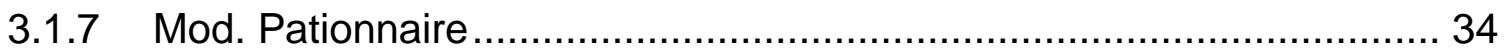

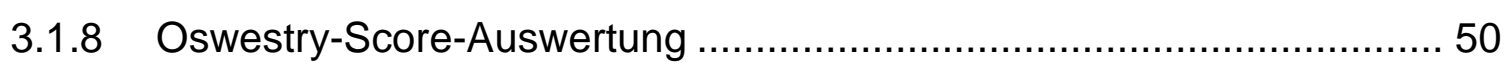

3.2 Radiologische Ergebnisse ........................................................... 52

3.2.1 Schraubenlockerungen ........................................................... 52

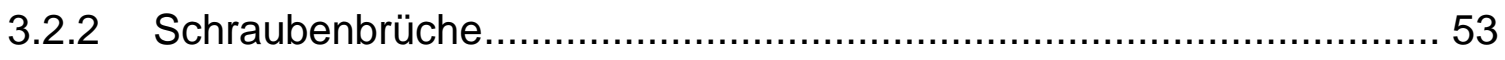

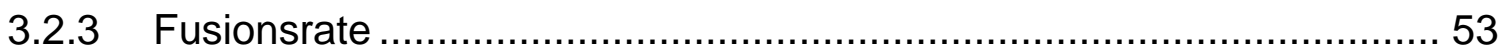

3.2.4 Anschlussinstabilitäten............................................................. 54

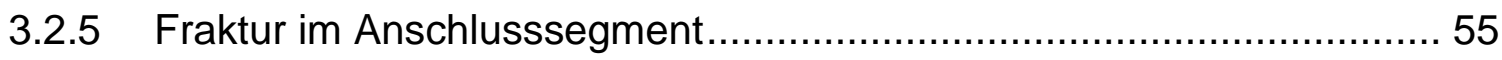


3.3.1 Einfluss des Rauchens auf die Fusionsrate ....................................... 56

3.3.2 Einfluss des Alters auf die Fusionsrate ........................................... 57

3.3.3 Einfluss des Operationsumfanges auf das Auftreten einer

Anschlussinstabilität

3.3.4 Postoperativer Schmerzmittelbedarf bei Anschlussinstabilität im

Vergleich zum präoperativen Schmerzmittelbedarf 59

3.3.5 Einfluss des Rauchens auf das Auftreten einer Schraubenlockerung... 60

3.3.6 Einfluss einer Schraubenlockerung auf die Fusionsrate 61

3.3.7 Einfluss von Alter und / oder BMI auf Schraubenbruch, -lockerung, Anschlussinstabilität, Fusion, Schmerzmittelbedarf 63

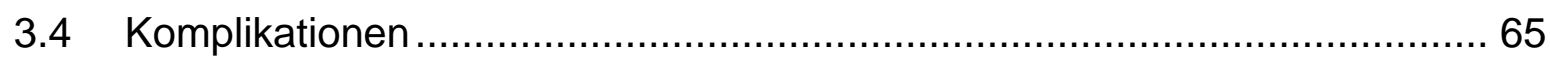

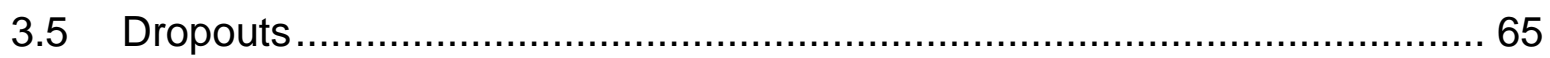

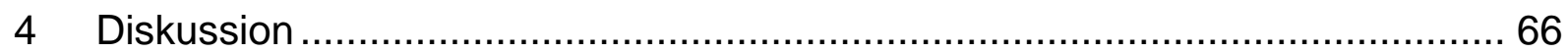

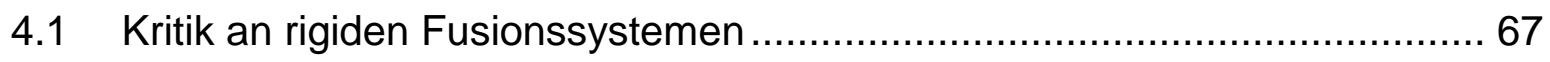

4.1.1 Anschlussinstabilitäten und -frakturen........................................... 67

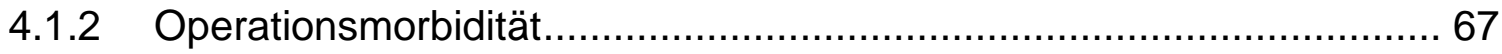

4.2 Die Entwicklung dynamischer transpedikulärer Systeme. Vorteile. ............ 68

4.3 Anforderung an dynamische transpedikuläre Systeme, Ergebnisse in der

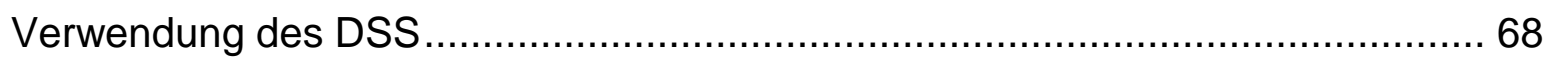

4.3.1 Schraubenlockerungsraten, Implantatbrüche....................................69

4.3.2 Biomechanische Veränderungen nach Fusion.................................. 70

4.4 Klinische Ergebnisse: Outcome-Beurteilung ….................................... 73

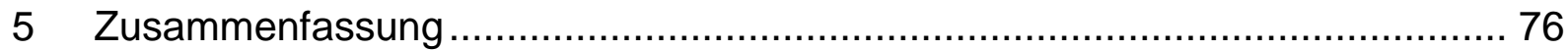




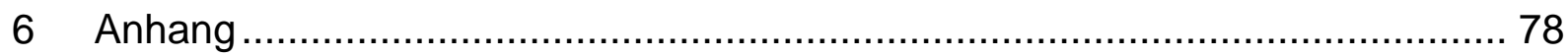

6.1 Fragebogen für die klinische Untersuchung …................................... 78

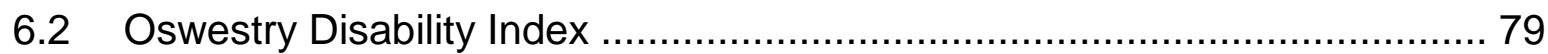

6.3 Modifizierter Pationnaire-Patienten-Fragebogen .................................... 81

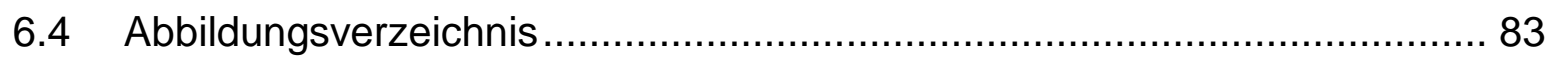

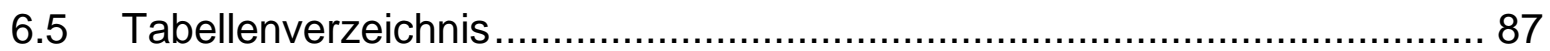

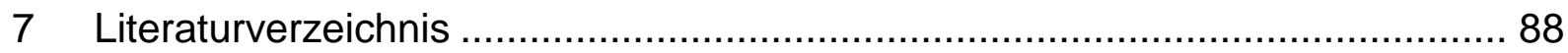




\section{Einleitung}

\subsection{Anatomische Aspekte und klinische Bedeutung der Instabilität der Lendenwirbelsäule}

Mit zunehmendem Alter steigt das Ausmaß der degenerativen Entwicklungen auch im Bereich der Lendenwirbelsäule. Die entstehenden biomechanischen Veränderungen können neben einer Affektion neuraler Strukturen auch zu einer progredienten Destabilisierung in dieser anatomischen Region führen. Bein- und Rückenschmerzen sind die Folge.

\subsubsection{Anatomische Grundlagen}

Die Lendenwirbelsäule besteht aus 5 Lendenwirbelkörpern, die über Bandscheiben, Facettengelenke und Bänder miteinander kommunizieren.

Die gesunde Bandscheibe erfüllt hierbei eine Art Pufferfunktion, die insbesondere axiale Belastungsspitzen abfedern soll. Sie besteht aus zwei verschiedenen Arten von Knorpelzellen und erhält ihre Elastizität über ihre Fähigkeit zur Wasserbindung. Über den Tag kommt es als Folge der axialen Belastung der Wirbelsäule durch Gehen, Stehen und Sitzen zu einer Flüssigkeitsverschiebung aus der Bandscheibe heraus, was einen Volumenverlust und somit eine Höhenminderung im Zwischenwirbelraum zur Folge hat. Eine Gesamtgrößenreduktion des Menschen am Abend ist die Folge.

An den Seiten der Wirbelkörper befinden sich die kleinen Wirbel- oder Facettengelenke. Ihre Aufgabe liegt vor allen Dingen in der Einschränkung des rotatorischen, extensorischen wie translationalen Bewegungsumfangs in der anteriorposterioren Richtung (Yang und King 1984, Haberl et al. 2004, Adams und Hutton 
1983). Wie alle anderen Gelenke des Körpers werden sie von einer Gelenkkapsel geschützt, die zum Erhalt eines physiologischen Gelenkmilieus beiträgt.

Als Folge der Höhenminderung im Zwischenwirbelraum nun entwickelt sich zum Abend eine leichte Destabilisierung im Bereich der Facettengelenke, die durch die Regeneration und somit Rehydrierung der Bandscheiben über die Nacht und die im Liegen erzielte Aufhebung der axialen Belastung bis zum Morgen wieder nivelliert wird.

Mit den Jahren und der damit in Verbindung stehenden Degeneration verlieren die Bandscheiben zunehmend ihre Fähigkeit zur Rehydrierung, ein Verlust der Wasserbindungsfähigkeit und somit Elastizität tritt ein. Die Folge ist ein progredienter und vor allen Dingen dauerhafter Verlust an Stabilität der Wirbelsäule.

Der folgende Höhenverlust führt im Weiteren zu einer Stellungsänderung der Facettengelenke, die eine arthrotische Deformierung und Hypertrophie zur Folge hat, - eine Erkenntnis, die bereits für andere Gelenke des Körpers als natürlicher Verlauf getroffen wurde. Eine idiopathische Facettengelenksarthrose kann einen ähnlichen Verlauf nehmen.

\subsubsection{Stadien der Degeneration}

In der Stadieneinteilung der klinischen Manifestation degenerativer Veränderungen an der Lendenwirbelsäule beschrieb Kirkaldy-Willis 3 Stufen:
1. Zeitweise Dysfunktion
2. Instabile Phase und
3. Die (Re-) Stabilisation.

Als klinisches Zeichen der Instabilität definierte er hierbei einen Zustand, bei dem der Patient mit Rückenschmerzen unter der leichtesten Belastung von milder 
Symptomatik zu schweren Schmerzzuständen wechselt (Kirkaldy-Willis und Farfan 1982).

In der pathoanatomischen Betrachtung entsteht eine erhöhte abnormale Beweglichkeit im betroffenen Gelenk, das in der Folge wiederum eine Reaktion auf die Verletzung zeigt. Hierbei zeigen Facettengelenke die gleichen Veränderungen wie andere Gelenke des Körpers: Je nach Ausmaß der Beeinträchtigung kommt es zu subchondralen Frakturen, Gelenkkörperformationen, zu entzündlichen Veränderungen mit exsudatvermittelter periartikulärer Fibrose (Troum und Crues 2004, Yamamoto et al. 1989).

Ein Großteil dieser Veränderungen lässt sich auf die als Folge der Bandscheibendegeneration veränderte Lastverteilung zurückführen. Unter der degenerativ bedingten Höhenreduktion der Bandscheibe im Alter kommt es zu erhöhten Scherkräften an den Facettengelenken, die, wie zuvor dargestellt, mit gelenktypischen Veränderungen reagieren und zur Zunahme der Instabilität und damit von Rücken- und Beinschmerzen beitragen können (Krismer et al. 2000, Wilke et al. 2009).

Altersbedingte Veränderungen der angrenzenden Bandstrukturen führen ebenso zu dieser pathophysiologischen Verschlechterung der biomechanischen Leistungsfähigkeit der Wirbelsäule (Kirkaldy-Willis und Farfan 1982).

\subsubsection{Klinische Bedeutung der degenerativen Instabilität}

Erschwerend kommt im Falle der Facettengelenke deren anatomische Beziehung zum Spinalkanal hinzu. In der Regel entwickelt sich im Zuge der Degeneration eine zunehmende Facettengelenkshypertrophie, die einen raumfordernden Effekt auf den Duralschlauch ausübt - eine spinale Stenosierung mit entsprechender Klinik - die Claudicatio spinalis - entsteht.

Verstärkt wird die Einengung des Rückenmarkskanals noch durch die im Höhenverlust der Bandscheibe begründete Auffältelung des gelben Bandes (Ligamentum flavum) ebenso wie durch die im Falle eines degenerativen 
Wirbelgleitens (Pseudospondylolisthese) resultierende Verschiebung der Raumvolumina des Spinalkanals auf Segmentebene.

Eine schmerzbedingte Einschränkung der Gehstrecke mit gegebenenfalls zusätzlichen neurologischen Symptomen wie Taubheitsgefühlen, Parästhesien, Lähmungen und vegetativen Störungen ist die Folge.

$\mathrm{Ob}$ und wie weit diese Veränderungen auch symptomatisch werden, ist hinsichtlich der genauen ätiologisch-anatomischen Zuordnung bislang nicht geklärt (Baur-Melnyk et al. 2006). Über die Schmerzhaftigkeit degenerativer Gelenkveränderungen im Sinne arthrotischer Umbauvorgänge (hier: Spondylarthrosen) besteht kein Zweifel. Unklar ist jedoch, ob und / oder in welchem Umfang die tatsächliche Einschränkung der Stabilität und die damit in Verbindung stehenden vergrößerten Bewegungsradien im Bereich der kleinen Wirbelgelenke zum klinischen Symptom des Schmerzes führen.

Ebenfalls nicht quantifizierbar ist der Einfluss der Bandscheibendegeneration selbst oder der beteiligten Wirbelkörper (Osteochondrose) auf das Gesamtbeschwerdebild. Gleiches gilt für die myofasziale Seite (Lotz und Ulrich 2006).

In manchen Fällen schafft es die Wirbelsäule über eine zunehmende Verknöcherung eine Stabilität zu erreichen. Ausgehend von den Gelenken oder den Wirbelkörpern selbst können Knochenspangen und Verwachsungen entstehen (Spondylophyten), die zu einer knöchernen Fusion und somit Festigkeit führen.

Sollten sich diese physiologischen Abstützreaktionen nicht ausreichend entwickeln, verbleibt die relative Instabilität, die im fortgeschrittenen Stadium in ein degeneratives Wirbelgleiten (Pseudospondylolisthesis) übergehen kann (Kirkaldy-Willis und Farfan 1982). 


\subsection{Behandlung der Instabilität}

\subsubsection{Konservative Behandlung}

Im ersten Schritt in der Behandlung von Instabilitäten der Lendenwirbelsäule kommen konservative Konzepte wie Ruhigstellung, aber auch gezieltes Hypertrophietraining der Rumpf- und Rückenmuskulatur zum Einsatz. Hierbei kann über die Verwendung von teilimmobilisierenden Korsetten eine passive Aufrichtung der Wirbelsäule erzielt werden, was zur Besserung von verspannungsbedingten Beschwerden und in Einzelfällen auch zu knöcherner Konsolidierung im krankhaft veränderten Segment führen soll.

\subsubsection{Die operative Behandlung der Instabilität der Wirbelsäule}

In der Konzeptionierung einer operativen Behandlungsstrategie für die segmentale Instabilität der Wirbelsäule herrscht im Allgemeinen kein Konsens, weder für die Wahl des Operationsverfahrens noch für den Zeitpunkt. Auch die Indikationsstellung zur operativen Versorgung ist nicht vollständig geklärt (Serhan et al. 2007). Historisch traten nach Verfahren wie der autologen Knochentransplantation nach Wiltse transpedikuläre Verfahren in Erscheinung mit besseren Fusionsergebnissen und einer hinsichtlich des zu erwartenden Ergebnisses höheren Verlässlichkeit.

In der Betrachtung der rigiden Fusionen nun wurden negative Aspekte dieses Versorgungsverfahrens erkannt wie eine erhöhte Rate an Instabilitäten und Stressfrakturen im kranialen Anschlusssegment. Langzeitergebnissen nach rigider Fusion hatten den Verdacht auf einen ursächlichen Zusammenhang zwischen rigider Fusion $\left(360^{\circ}\right.$-Fusion, interkorporelle Spondylodese) und einer zunehmenden 
Degeneration in angrenzenden Segmenten nahegelegt (Kumar et al. 2001, Markwalder und Wenger 2002).

Auch in der Gesamtbeurteilung der Operationsergebnisse musste konstatiert werden, dass hinsichtlich der Aufgabenstellung eines schmerzlindernden Verfahrens nur in 50-70\% der Fälle gute Ergebnisse gesehen werden (Boos und Webb 1997, Turner et al. 1992, Bothmann et al. 2008, Thomas et al. 2009).

Kompliziert wird die Einschätzung des Operationserfolges überdies durch die Beobachtung, dass offenbar auch im Falle von Pseudarthrosen Schmerzfreiheit bestehen kann und im Gegensatz hierzu bei erfolgreichen knöchernen Fusionen Beschwerden fortbestehen können (Sengupta und Herkowitz 2005, Fischgrund et al. 1997).

Hieraus entstand die Forderung nach Operationstechniken, die eine ausreichende Stabilität im operierten Segment ermöglichen, jedoch noch genügend Beweglichkeit erlauben, um die Degeneration im Anschlusssegment nicht in gleichem Maße zu begünstigen wie ein rigides System (Wilke et al. 2009). Solche Verfahren werden als Dynamische Systeme bezeichnet.

\subsubsection{Rigide Stabilisierungstechniken}

Um das Fortschreiten der Instabilität zu verhindern, standen zunächst nichtinstrumentierte Verfahren wie die autologe dorsolaterale Knochentransplantation nach Wiltse zur Verfügung.

Im Weiteren wurden Instrumentationen entwickelt, die im Wesentlichen auf einem transpedikulären Schraubensystem beruhten, ergänzt durch eine interkorporelle Spondylodese (Wirbelfusion) durch autologen Knochen oder später Platzhalter aus Fremdmaterialien (Cages). Hierdurch konnte eine kontinuierliche Verbesserung der Fusionsraten erreicht werden (Bridwell et al. 1993, Mardjetko et al. 1994, Yaun et al. 1994, Rechtine et al. 1996, Fernandéz-Fairen et al 2007, Johnston et al. 1990). 


\subsubsection{Dynamische Stabilisierungssysteme}

Die Aufgabe der dynamischen Systeme ist, die pathologische segmentale (Über-) Beweglichkeit zu reduzieren und die Last im Bereich der Bandscheiben sowie der Wirbelgelenke einzuschränken (Mandigo et al. 2007, Schmidt et al. 2008). Hierbei entsteht die Indikation zur Verwendung des Systems in der heutigen Betrachtung im Allgemeinen zeitlich vor einem Zeitpunkt zur sogenannten rigiden oder $360^{\circ}$-Fusion. Ziel des dynamischen Versorgungskonzeptes ist, die Beweglichkeit im behandelten Segment weitgehend zu erhalten und zwar zu einem Zeitpunkt, an dem das Ausmaß der Degeneration noch eine weitgehende segmentale Funktionalität verspricht. Spätere Folgen der degenerativen Instabilität wie Entstehung oder Zunahme translationaler Fehlstellungen der Wirbelsäule sollen somit durch die rechtzeitige Intervention verhindert werden.

Man unterscheidet im Wesentlichen interkorporelle (Bandscheibenprothesen), interspinöse und pedikelschraubenbasierte Verfahren:

\section{- Bandscheibenprothesen unterschiedlicher Hersteller}

\section{- Interspinöse Spreizer}

Arbeitsprinzip der interspinösen Spreizer ist die Entlastung der Facettengelenke und eine Limitierung des Bewegungsumfanges (ROM) im Bewegungssegment.

- Das interspinöse U (Le U, später Coflex. Fa. Paradigm Spine New York)

- X-Stop (St. Francis Medical Technologies, Californien)

- Wallis Interspinous Implant (Abbott Spine, Texas)

- DIAM (Device for Intervertebral Assisted Motion) (Medtronic, Tennessee) 
- The Artificial Ligament (Liganove Spine Ligament WSH, Cousin Biotech, Frankreich)

- Shape Memory Implant (CJSC KIMPF Company, Moskau)

\section{- Pedikelschraubenbasierte dynamische Fusionssysteme}

- Dynesys (Zimmer Spine, Illinois)

- Cosmic MIA (Ulrich Medical, Ulm)

- Softflex System (Globus Medical, Arizona)

- Graf Ligament (SEM Sarl, Frankreich)

- Isobar TTL (Scient'x USA, Florida)

- DSS Dynamisches Schraubensystem (Bricon, Schweiz)

- DSS -I (Abbott Spine, Texas)

- BioFlex Spring Rod Pedicle Screw System (Bio Smart Ltd., Korea).

(Khouier et al. 2007, Kim DH et al. 2006)

\subsection{Problemstellung}

Dynamische transpedikuläre Versorgungsstrategien erfordern aufgrund ihres geringen historischen Alters von bislang etwa 20 Jahren des klinischen Einsatzes weitere wissenschaftliche Evaluation (Martin et al. 2007). Die aktuelle Datenlage zur Indikationsstellung für pedikelschraubenbasierte dynamische Fusionssysteme ist unzureichend (Grob et al. 2005, Lawthorne et al. 2009).

Unklar ist, ob die Rate an systembedingten Komplikationen wie Schraubenlockerungen oder -brüchen sowie Instabilitäten im Anschlusssegment im Vergleich zu rigiden Fusionsstrategien erhöht ist oder ob, bei geeigneter Fragestellung, die dynamische Verschraubung ein gleich- oder gar höherwertiges Ersatzverfahren darstellt bei ja gegeben reduzierter Operationsmorbidität aufgrund eines geringeren Zugangstraumas, kürzerer OP-Dauer und geringerer Kosten im Vergleich zur sogenannten instrumentierten $360^{\circ}$-Fusion. 
Einleitung

\subsection{Fragestellung und Zielsetzung}

Ziel der Studie ist eine klinische und radiologische Evaluierung des Outcomes von Patienten, die in einer konsekutiven Reihe von 120 Fällen in verschiedenen Indikationsgruppen bei degenerativer Spondylolisthese mit dem dynamischen Fusionssystem DSS der Firma Bricon versorgt wurden.

Um die Verwendbarkeit des DSS Dynamischen Schraubensystems in der Behandlung lumbaler Instabilitäten zu klären, soll:

- eine Auswertung der Schraubenlockerungsrate erfolgen,

- das Auftreten von Stressfrakturen im Anschlusssegment geprüft werden,

- eine klinische Einschätzung des subjektiv beurteilten Operationserfolges anhand einer patientenorientierten Fragebogenevaluation durchgeführt werden. 


\section{Material und Methoden}

\subsection{Studiendesign}

Bei der vorliegenden Nachuntersuchungsstudie handelt es sich um eine Datenerhebung im Rahmen einer prospektiven Betrachtung. Sie zeigt Ein-JahresErgebnisse dynamisch bzw. kombiniert dynamisch-rigide fusionierter Patienten. Die radiologische Bewertung des Implantats und die Selbsteinschätzung des Patienten bezüglich seiner Lebenssituation nach der vorgenommenen Operation sind die zentralen Elemente der Studie.

\subsubsection{Patientenselektion}

In die Studie wurden alle Patienten einbezogen, die im Zeitraum vom 03.07.2007 bis 15.12.2008 von den Operateuren der Neurochirurgie Osthessen GbR eine dynamische oder auch kombiniert dynamisch und rigide Fusion der Lendenwirbelsäule erhielten.

Als Einschlusskriterium galt hierbei die Implantation des Bricon DSS-Systems, entweder als rein dynamische Fusion oder in Kombination mit einer rigiden Fusion (sog. Topping off) (Kim YS et al. 2007). Ausgeschlossen aus der Studie wurden alle Patienten, die eine rein rigide Fusion erhielten. Ebenso wurden Patienten, die mit einem dynamischen System einer anderen Firma versorgt wurden, in dieser Studie nicht berücksichtigt.

Indikation zur Implantation des Systems waren degenerative Spondylolisthesen mit und ohne spinale Stenosierung, die unter konservativer Therapie über einen Zeitraum von mindestens 6 Monaten keine ausreichende Besserung der Beschwerdesymptomatik erfahren hatten. 
Material und Methoden

Es wurden 120 Patientinnen und Patienten telefonisch $\mathrm{zu}$ einem Nachuntersuchungstermin eingeladen. Von diesen beteiligten sich 99 an der vorliegenden Studie - somit ein Rücklauf von $82,5 \%$.

10 Patienten waren zwischenzeitlich unbekannt verzogen, 2 Patienten im Verlauf des ersten postoperativen Jahres verstorben, 6 Patienten verweigerten die Teilnahme an der Nachuntersuchung und 3 Patienten erhielten während der vergangenen 12 postoperativen Monate eine Revisionsoperation, in der das dynamische System entfernt wurde.

Die Geschlechterverteilung wies eine Quote von 68 weiblichen und 52 männlichen Studienteilnehmern auf. Das Alter des Patientenkollektivs umfasste den Bereich von 32 Jahren bis 87 Jahren und führte somit zu einem Durchschnittsalter von 65,5 Jahren.

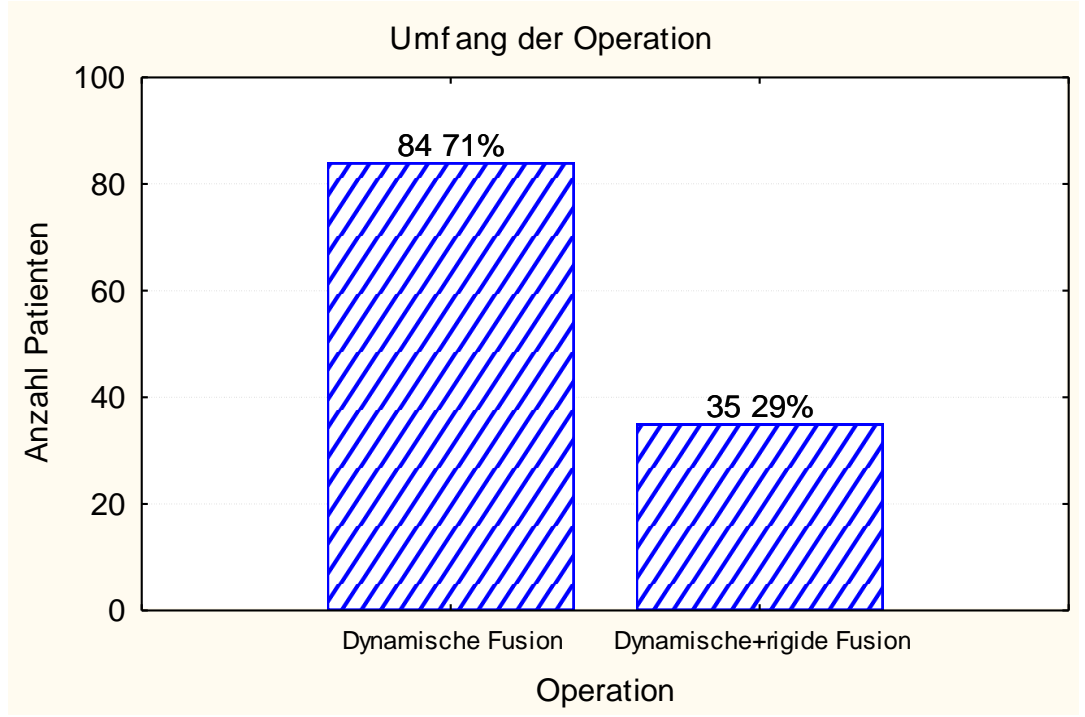

Abbildung 1: Umfang der Operation 


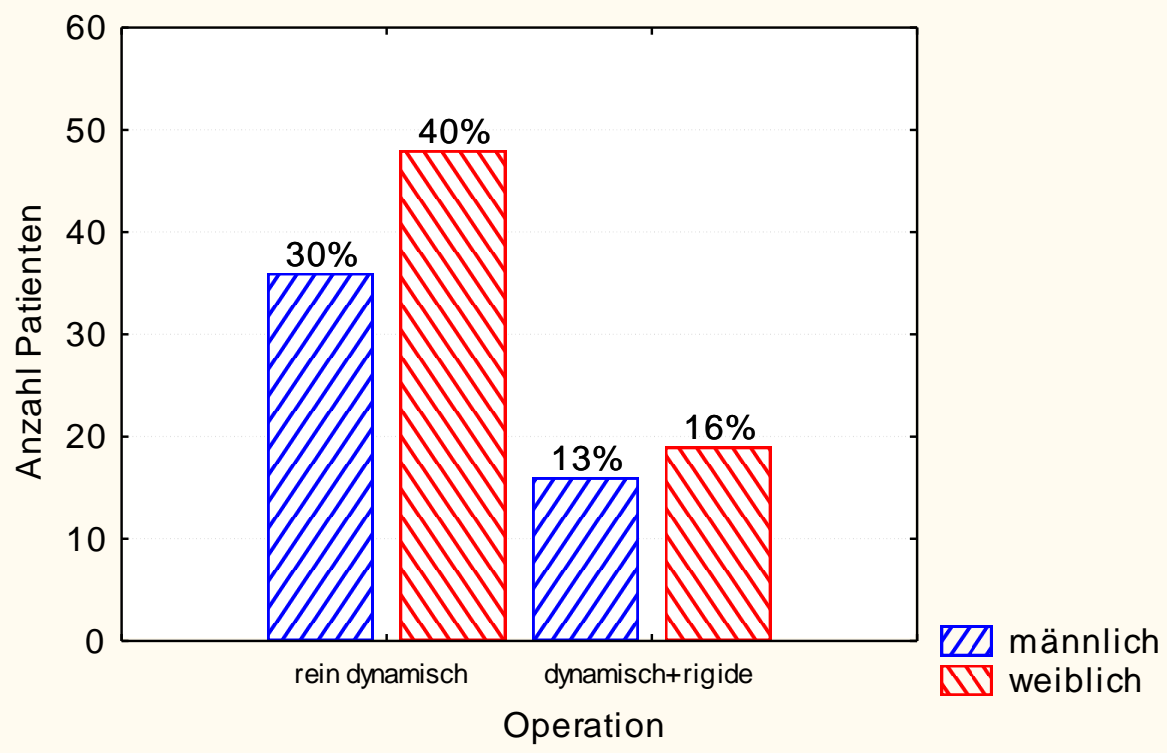

Abbildung 2: Geschlechterverteilung je OP-Umfang

Der Nachuntersuchungstermin lag im Schnitt bei 12,6 Monaten postoperativ, wobei die angefertigten CT- und Funktionsröntgenaufnahmen im Schnitt nach 11,4 Monaten postoperativ entstanden sind.

Die operierten Höhen umfassten LWK2 - SWK1.

79 Patienten wurden nur in 1 Etage operiert, 33 Patienten in 2 Etagen und 7 Patienten in 3 Etagen.

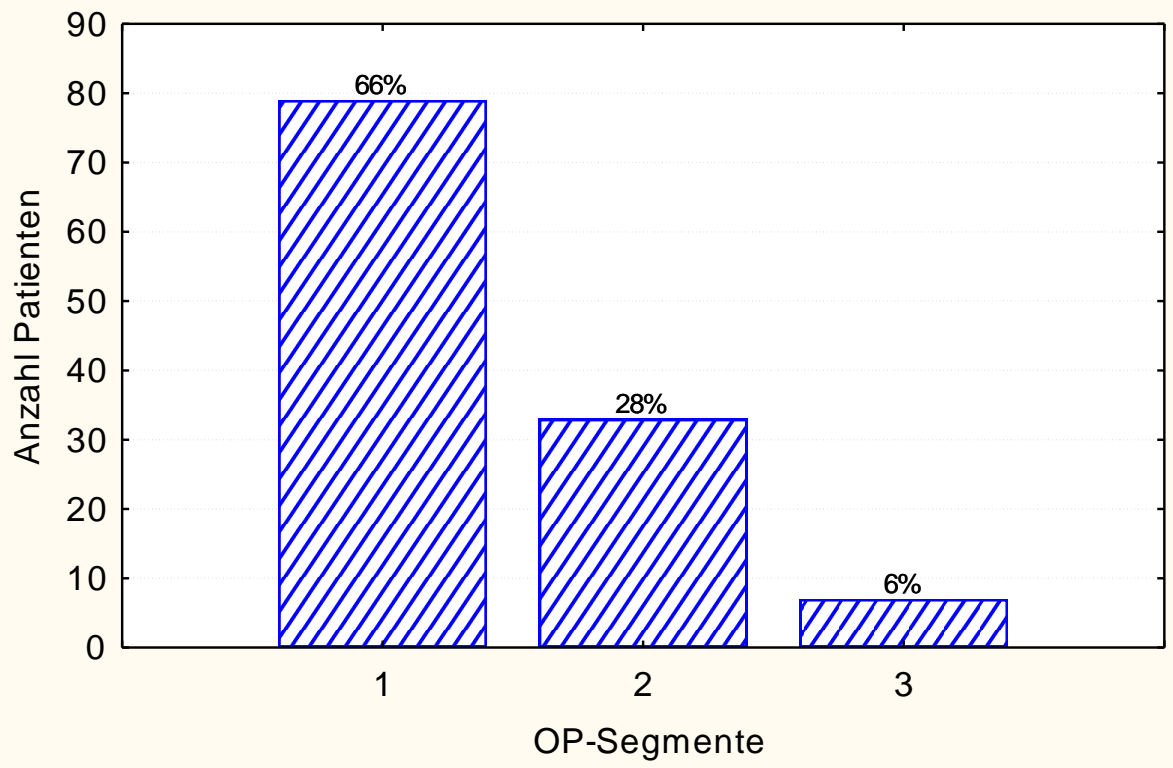

Abbildung 3: Anzahl der betroffenen Segmente 
Von den nachuntersuchten Patienten erhielten 84 eine rein dynamische Fusion, Geschlechterverhältnis 36:48 (m:w) Patienten, und 35 Patienten eine kombiniert dynamisch und rigide Fusion, Geschlechterverhältnis 16:19 (m:w) Patienten.

Die Aufteilung der operativ versorgten Höhen stellt sich wie folgt dar. Rein dynamische Fusion: $L 2 / 3=1$ Patient, L3/4 = 5 Patienten, L4/5 = 69 Patienten, L5/S1 = 4 Patienten und L3/4+L4/5 = 5 Patienten.

Kombinierte Fusion, d.h. dynamisch + rigide:

dynamische Fusion: $L 2 / 3=2$ Patienten, $L 3 / 4=16$ Patienten, $L 4 / 5=16$ Patienten, $\mathrm{L} 5 / \mathrm{S} 1=$ kein Patient, $\mathrm{L} 2 / 3+\mathrm{L} 3 / 4=1$ Patient und $\mathrm{L} 3 / 4+\mathrm{L} 4 / 5=$ kein Patient; rigide Fusion: $L 4 / 5=13$ Patienten, L5/S1 = 16 Patienten, L3/4+L4/5 = 2 Patienten und L4/5+L5/S1 = 4 Patienten.

\begin{tabular}{|c|c|}
\hline $\begin{array}{c}\text { Dynamische Fusion } \\
\text {-Höhe- }\end{array}$ & Patientenanzahl \\
\hline $\mathrm{L} 2 / 3$ & 1Patient \\
\hline $\mathrm{L} 3 / 4$ & 5 Patienten \\
\hline $\mathrm{L} 4 / 5$ & 69 Patienten \\
\hline $\mathrm{L} 5 / \mathrm{S} 1$ & 4 Patienten \\
\hline $\mathrm{L} 2 / 3+\mathrm{L} 3 / 4$ & Kein Patient \\
\hline $\mathrm{L} 3 / 4+\mathrm{L} 4 / 5$ & 5 Patienten \\
\hline $\mathrm{L} 4 / 5+\mathrm{L} 5 / \mathrm{S} 1$ & Kein Patient \\
\hline
\end{tabular}

Tabelle 1: Höhenverteilung Dynamische Fusion 


\begin{tabular}{|c|c|c|}
\hline $\begin{array}{c}\text { Patientenanzahl } \\
\text {-dynamisch- }\end{array}$ & Höhe & $\begin{array}{c}\text { Patientenanzahl } \\
\text {-rigide- }\end{array}$ \\
\hline 2 Patienten & $\mathrm{L} 2 / 3$ & Kein Patient \\
\hline 16 Patienten & $\mathrm{L} 3 / 4$ & Kein Patient \\
\hline 16 Patienten & $\mathrm{L} 4 / 5$ & 13 Patienten \\
\hline Kein Patient & $\mathrm{L} 5 / \mathrm{S} 1$ & 16 Patienten \\
\hline 1Patient & $\mathrm{L} 2 / 3+\mathrm{L} 3 / 4$ & Kein Patient \\
\hline Kein Patient & $\mathrm{L} 3 / 4+\mathrm{L} 4 / 5$ & 2 Patienten \\
\hline Kein Patient & $\mathrm{L} 4 / 5+\mathrm{L} 5 / \mathrm{S} 1$ & 4 Patienten \\
\hline
\end{tabular}

Tabelle 2: Höhenverteilung Dynamisch plus rigide Fusion

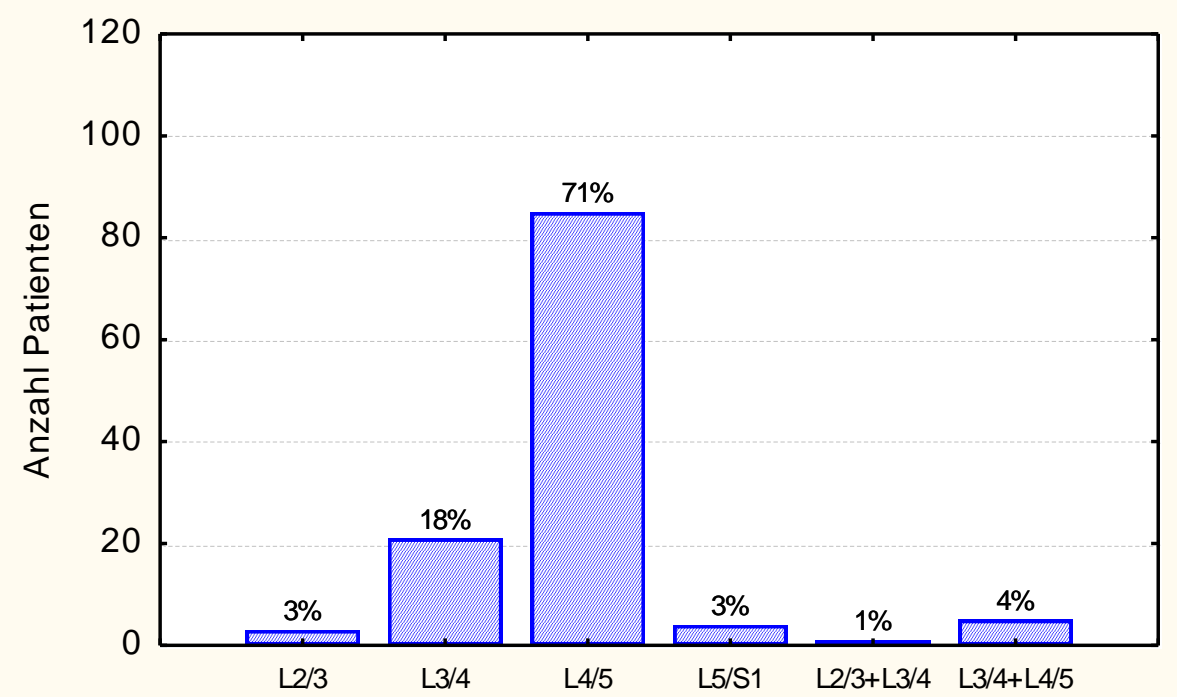

Abbildung 4: Höhenverteilung aller Dynamischen Systeme inklusive Topping off 


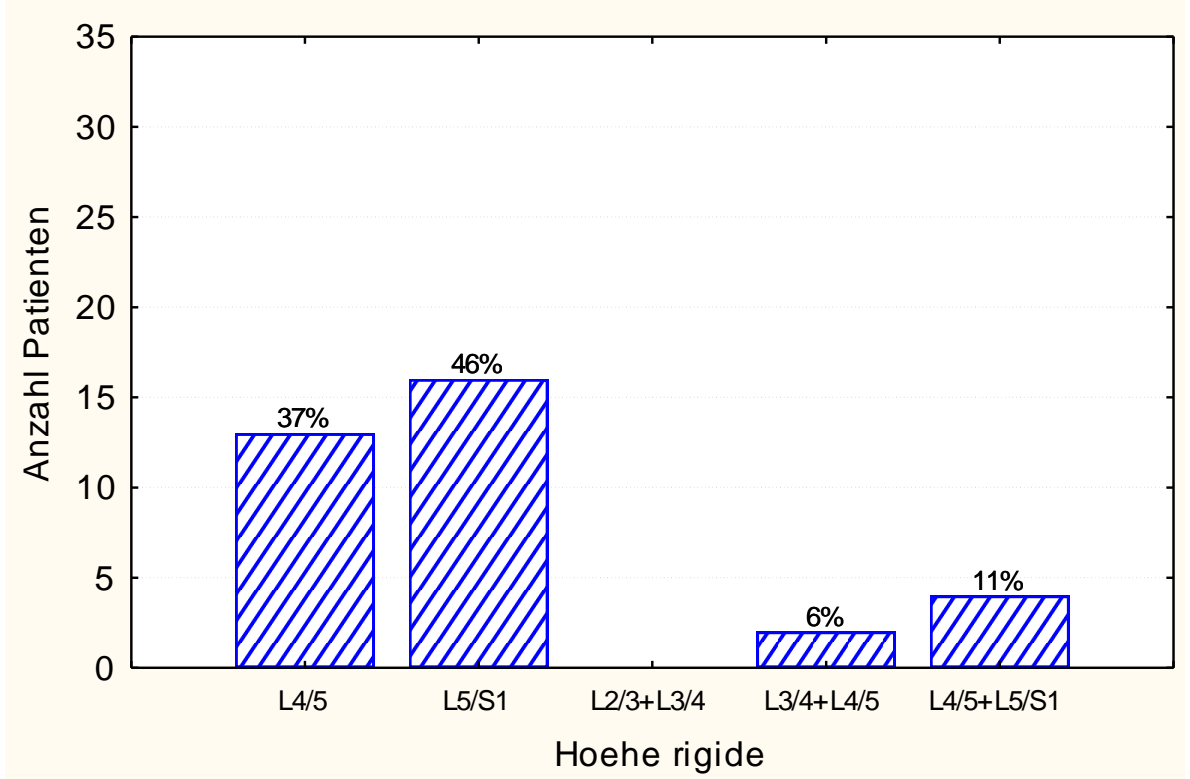

Abbildung 5: Höhenverteilung aller rigiden Fusionen mit Topping off im Anschlusssegment

Zusätzlich zur Fusionsoperation erhielten 87 Patienten eine Dekompression einer Spinalkanalstenose.

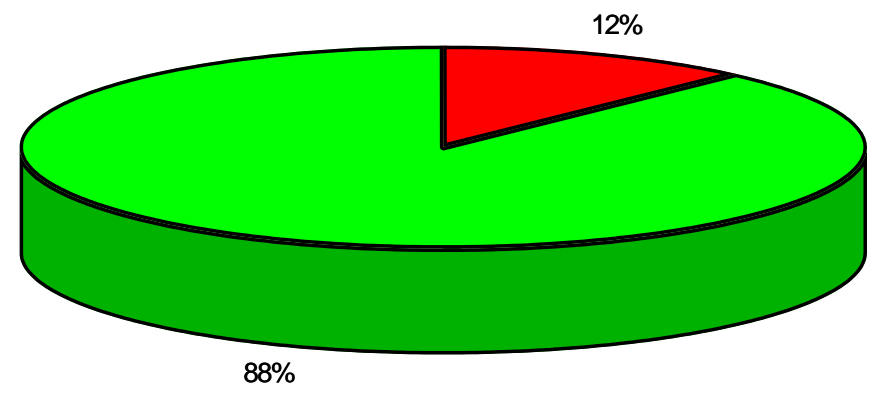

Dekompression

Keine Dekompression

Dekompression

Abbildung 6: Quote der dekomprimierten Spinalkanalstenosen 


\subsubsection{Klinische Nachuntersuchung:}

Untersuchungsintervalle

Die Patienten wurden zu routinemäßigen Nachuntersuchungsterminen 6 Wochen postoperativ, sowie 6 und 12 Monate postoperativ einbestellt. Bei den ersten beiden Nachuntersuchungsterminen wurde die Implantatlage anhand von anterior-posterior (a.p.)-Röntgenaufnahmen der LWS bewertet. Erst bei der 1-Jahres-postOP-Kontrolle wurde nochmals ein Computertomogramm angefertigt. Zudem wurden die Patienten bei allen Terminen klinisch untersucht und gebeten, ihre momentane Situation einzuschätzen. Mit Hilfe von 3 Fragebögen sollte diese Einschätzung für den Untersucher auch sichtbar gemacht werden. Lediglich die erhobenen Daten der Abschlusskontrolle, die um den 12. postoperativen Monat lagen, gehen in diese Dissertation ein.

Die abgefragten Kriterien wurden anhand der folgenden Fragebögen evaluiert:

\subsubsection{Klinischer Fragebogen (s. Anhang)}

Im Rahmen der klinischen Nachuntersuchung wurden anhand eines spezifischen Fragebogens (siehe Anhang) folgende Kriterien abgefragt:

- Welches Ausmaß hatte die Operation ?

- Wurde eine Spinalkanalstenose dekomprimiert?

- Alter des Patienten/der Patientin

- Größe und Gewicht der Patienten, um den Body-Mass-Index zu ermitteln

- Wurde der Patient bereits zuvor an der Wirbelsäule operiert? Und wenn, wie häufig? 
- Hat sich der Schmerzmittelbedarf im Vergleich zur präoperativen Situation verändert?

- Welcher OP-Zugang wurde gewählt? Wie stellen sich die Narben dar?

- Gab es intraoperative Komplikationen?

- Wurde der Patient/die Patientin in den vergangenen 12 postoperativen Monaten ein weiteres Mal an der Wirbelsäule operiert?

Nach der ausführlichen Anamnese folgte die gründliche symptombezogene neurologische Untersuchung der Patienten.

\subsubsection{Oswestry-Score (s. Anhang)}

Der Oswestry Disability Questionnaire ist ein Fragebogen, der von den Patientinnen/Patienten ausgefüllt wird. Er soll die, von den Patienten empfunden Behinderungen aufgrund von Rückenschmerzen abbilden. Entwickelt wurde der Fragebogen 1976 von John O’Brien und 1980 dann von einem orthopädischen Chirurgen (Stephan Eisenstein) und einer Ergotherapeutin (Judith Couper) publiziert. (Fairbank et al. 1980, Fairbank und Pynsent 2000, Fairbank 2007, Pratt et al. 2002) Im Verlauf hat man aus der ursprünglichen Version 3 weitere Versionen entwickelt und den Bogen in verschiedene Sprachen übersetzt. In der vorliegenden Dissertation wurde eine deutsche Übersetzung der Version 1 des Oswestry-DisabilityQuestionnaires verwendet. Die Patienten sollen bei jeder Frage immer nur eine Antwort, die für sie am ehesten zutreffende, ankreuzen. Sie sollen hierbei selbst einschätzen, inwieweit sie bei der Durchführung einer Aufgabe oder Tätigkeit beeinträchtigt sind. Pro Frage kann man 5 Punkte erreichen. D.h., wenn alle Fragen beantwortet wurden, erhält man eine Maximalpunktzahl von 50 Punkten. Danach ermittelt man den prozentualen Anteil der erreichten Punkte von der Maximalpunktzahl. Daraus ergibt sich dann folgende Auswertung: 
$0-20 \%=$ minimale Behinderung

$20-40 \%=$ mäßige Behinderung

$40-60 \%=$ starke Behinderung

$60-80 \%=$ invalidisierend

$80-100 \%=$ bettlägerig

Da 35 Patienten die achte Frage nach der Beeinträchtigung ihres Sexuallebens nicht beantworten konnten oder wollten, wurde die erreichbare Maximalpunktzahl bei diesen Patienten auf 45 Punkte, d.h. 50 Punkte minus der maximalen Punktzahl für Frage 8 reduziert. Somit kann man dann ebenfalls den prozentualen Anteil an der korrigierten Maximalpunktzahl berechnen und diese Patienten statistisch berücksichtigen. Nach dieser Korrekturformel wurde auch verfahren, wenn Patienten eine andere Frage nicht beantworten konnten bzw. wollten oder, wenn mehr als eine Frage nicht beantwortet wurde (Anleitung des Verfassers).

\subsubsection{Modifizierter Pationnaire-Bogen (s. Anhang)}

Der modifizierte Pationnaire-Fragebogen wurde so verändert, dass es den Patienten möglich war, ihre Beschwerden im Vergleich präoperativ zu postoperativ zu bewerten. Der Fragebogen ermöglicht die interaktive Anamnese des Patienten. Befragte sollen u.a. anhand der NAS (Numerische Analogskala) ihre empfundene Schmerzstärke (Rücken- und Beinschmerzen) angeben. Des Weiteren werden Symptome und empfundene Einschränkungen im prä-/postoperativen Vergleich abgefragt. Am Ende des Bogens können die Patienten ihre Zufriedenheit mit der individuellen postoperativen Gesamtsituation bewerten und werden zudem nach ihrer erneuten OP-Motivation befragt. Durch den Fragebogen wird die objektive Messung von Beschwerden, Behinderungen und des Befindens des Patienten ermöglicht. Eine kurze Besprechung des zuvor selbständig bearbeiteten Fragenkatalogs soll beim Befragten das Gefühl der Einbeziehung in die Evaluation fördern. 


\subsubsection{Radiologische Nachuntersuchung:}

Die Nachuntersuchungsintervalle folgten dem in der Klinik für Wirbelsäulenchirurgie der Main-Kinzig-Kliniken üblichen Muster nach lumbaler Instrumentation. Hierbei erfolgte nach 6 Wochen die erste Röntgenkontrolle der LWS mit der Frage nach nativradiologisch erkennbarer Implantatlockerung.

Nach Ablauf eines Jahres erfolgte die Abschlussuntersuchung mit CT der LWS und der Frage nach einer möglichen Fusion, Implantatlockerung im Detail (im Vergleich zur nativradiologischen Diagnostik höhere Sensitivität), Stressfraktur im Anschlusssegment oder einer Anschlussinstabilität als mögliche Folgen der Stabilisierung.

Als Lockerungszeichen wurden hierbei computertomografisch gesicherte Lysezonen (Resorptionssäume) um die eingebrachten Schrauben bewertet.

Ergänzt wurde diese Bildgebung im Weiteren durch eine LWS-Funktionsaufnahme in maximal möglicher Inklination und Reklination, um auf die Frage nach einer Anschlussinstabilität unter Belastung näher eingehen zu können. Als Instabilitätskriterien wurden hierbei eine Translationsbewegung von mehr als $3 \mathrm{~mm}$ oder $5-10^{\circ}$ Rotation in Seitneigung aufgefasst (Bambakidis et al. 2005). 
Material und Methoden

2.1.4 Beispiel Anschlussinstabilität

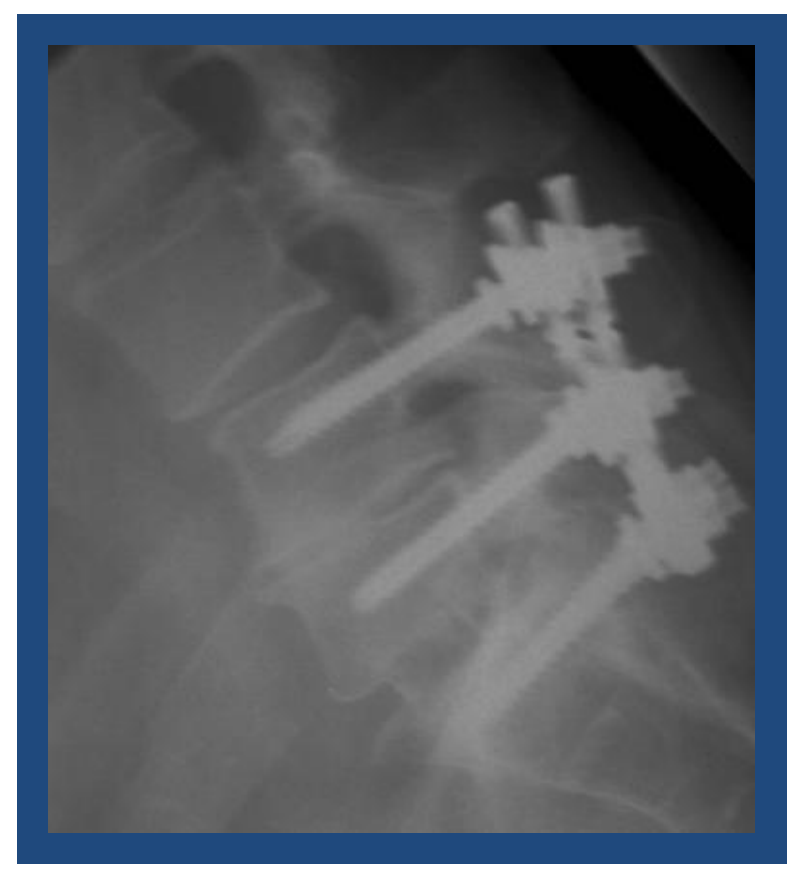

Abbildung 7: Anschlussinstabilität im Funktionsröntgen

2.1.5 Beispiel Anschlussfraktur

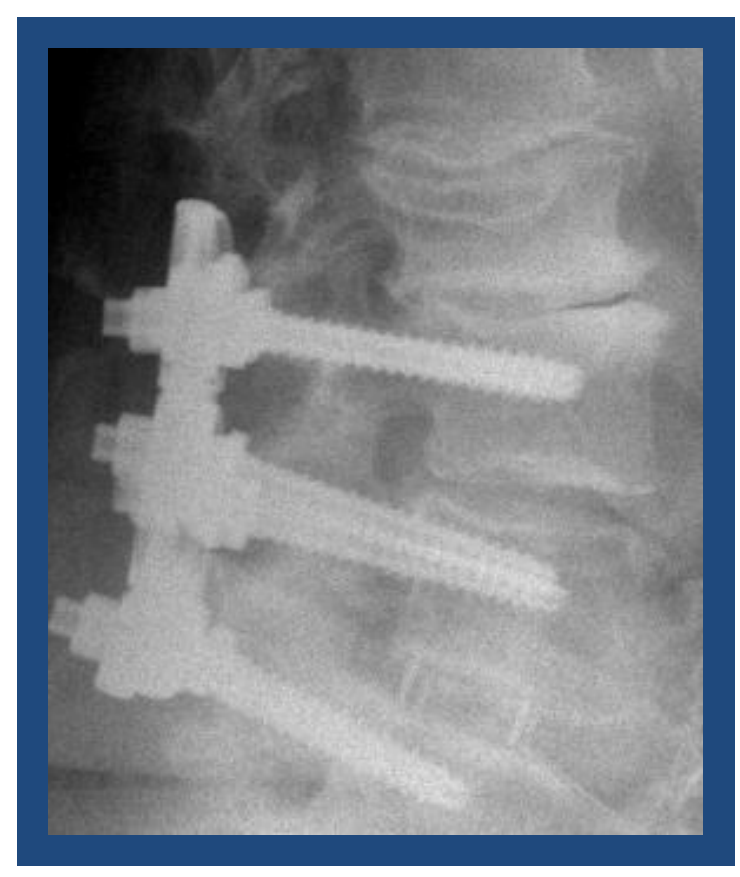

Abbildung 8: Anschlussfraktur 
Material und Methoden

2.1.6 Beispiel Schraubenlockerung

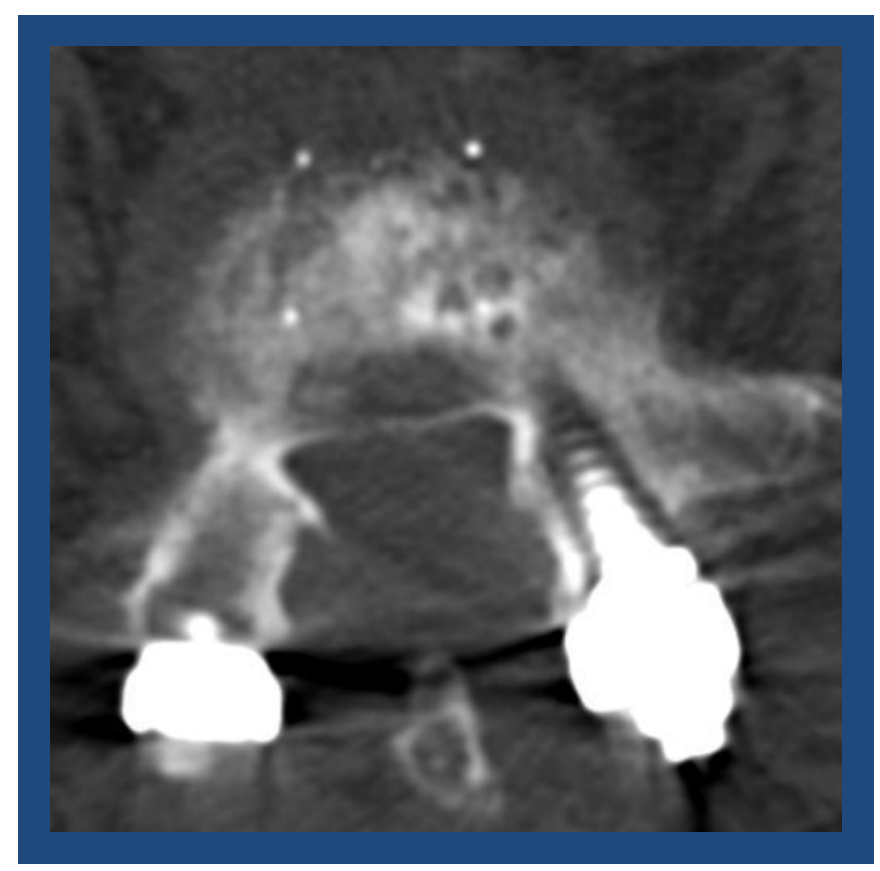

Abbildung 9: Schraubenlockerung links

2.1.7 Beispiel Schraubenbruch

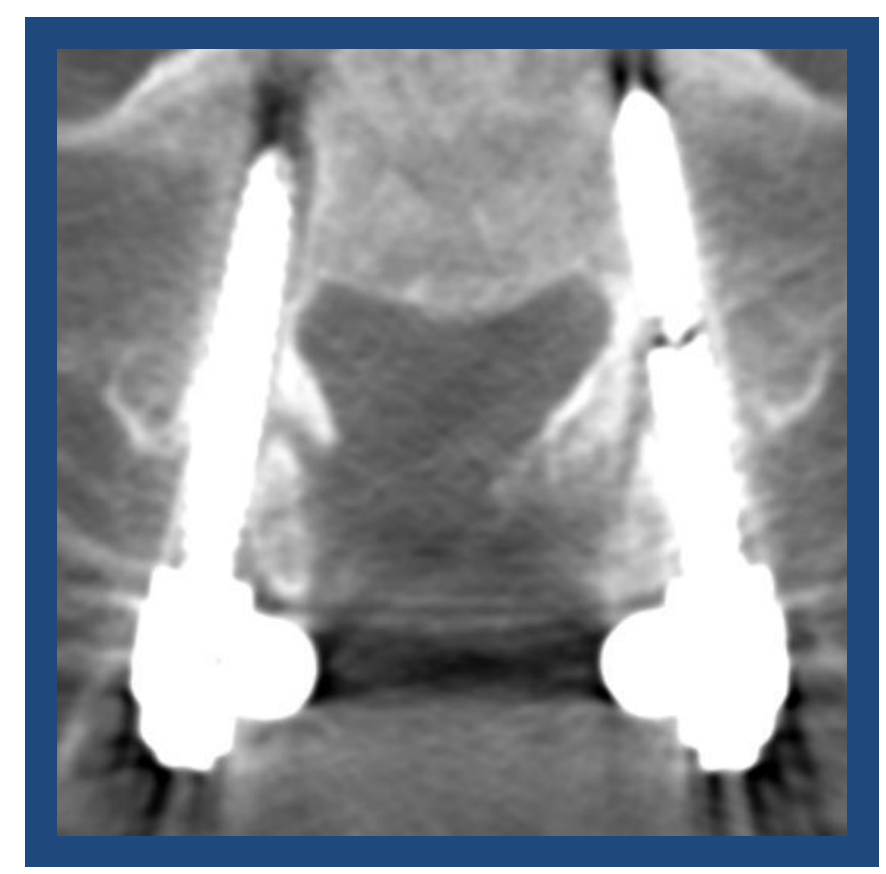

Abbildung 10: Schraubenbruch links 
Material und Methoden

2.1.8 Beispiel Knöcherne Fusion

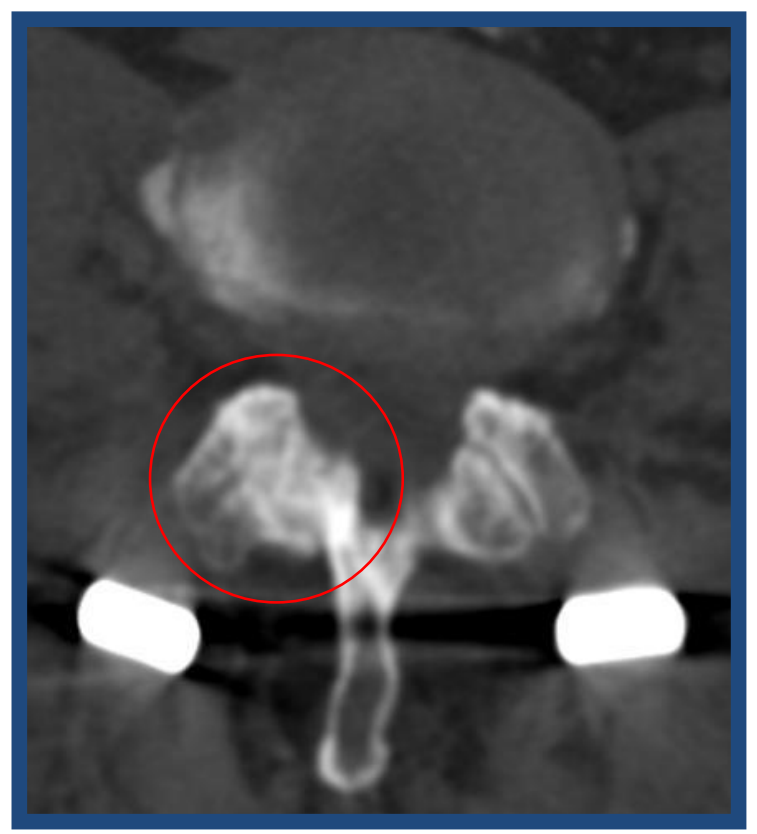

Abbildung 11: Gelenkfusion rechts 


\subsection{Das Bricon DSS}

Bei dem verwendeten Implantatsystem handelt es sich um das DSS (Dynamisches Schraubensystem) der Firma Bricon, Schweiz. Die dynamischen Schrauben sind eine Weiterentwicklung der semirigiden Schrauben, die nur in 1 Ebene kippbar sind und aus diesem Grund sehr sauber eingebracht werden müssen. D.h. es entsteht eine gewisse Operateurabhängigkeit. Da die dynamischen Schrauben in alle Richtungen kippbar sind, wird das Einbringen der Schrauben etwas erleichtert. Das Schrauben-Stangen-/-Platten-System ist aus Titan gefertigt, das sich über Jahrzehnte für Implantate bewährt hat. Durch 2 verschiedene Drehzentren in den beiden Pedikelschraubenköpfen pro Wirbelkörper wird die Rotation um die Wirbelsäulenachse stabil abgestützt, bei jedoch erhaltener Restbeweglichkeit des fixierten Segments. Die große Beweglichkeit in alle Richtungen durch das Kugelgelenk (20 Grad aus der Schraubenachse) erlaubt einen problemlosen, fehlertoleranten Einbau. Die Restbeweglichkeit in der Sagittalebene ist praktisch immer gegeben, egal in welcher Richtung die Schraube gesetzt wird. Eine Distraktion im Segment kann bei verbleibender Beweglichkeit der Wirbelkörper vorgenommen werden.

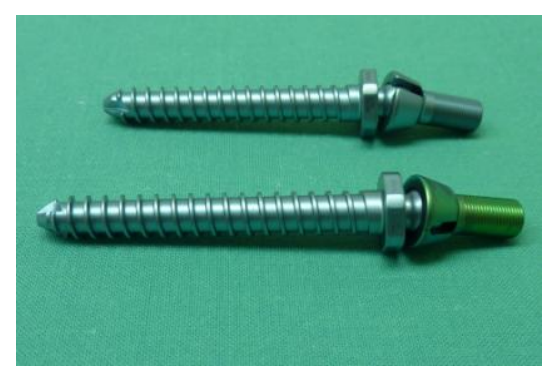

Abbildung 12: Rigide (hinten) und dynamische (vorne) Schrauben

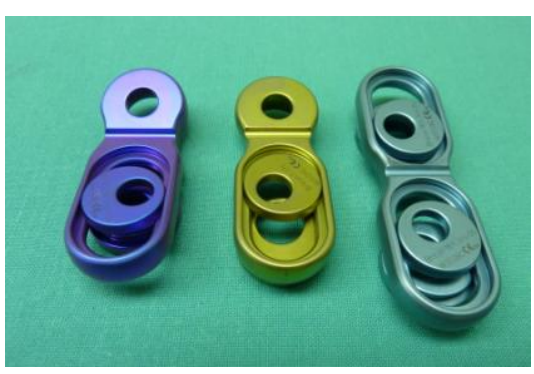

Abbildung 13: Platten des Bricon DSS

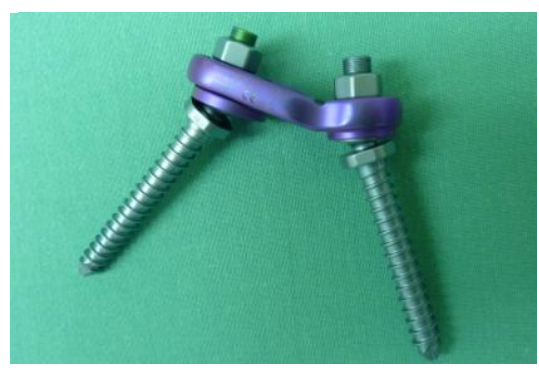

Abbildung 14: Dynamisches 


\subsection{Operationsablauf}

Die operativen Prozeduren werden untergliedert in offene und transmuskuläre Zugänge sowie eine Gruppe von Patienten, die zur dynamischen Fusion eine Dekompression einer spinalen Stenose erhielten sowie rein dynamische Fusionen.

\subsubsection{Offener Zugang}

Im Rahmen der offenen Operation erfolgt im Zugangsbereich über den Dornfortsätzen der betroffenen und angrenzenden Wirbel ein Mittellinienschnitt in einer Länge von etwa $12 \mathrm{~cm}$ im monosegmentalen Zugang, etwa $15 \mathrm{~cm}$ beim bisegmentalen Zugang und etwa $18 \mathrm{~cm}$ für Eingriffe über 3 Höhen. Es folgt die Darstellung der thorakolumbalen Muskelfaszie und nach deren Durchtrennung das bilaterale Abschieben der Muskulatur über das Gelenkniveau der Facetten hinaus. Hierauf werden nach ausgiebiger Blutstillung ein oder zwei Sperrer eingesetzt, die die Muskeln von der Wirbelsäule für die Dauer der Operation trennen.

\subsubsection{Transmuskulärer Zugang}

Für den transmuskulären Zugang erfolgt der Hautschnitt direkt über den Pedikeln, die im Vorfeld radiologisch markiert werden in einer Länge von etwa $3 \mathrm{~cm}$ beim monosegmentalen, etwa $5 \mathrm{~cm}$ beim bisegmentalen und etwa $10 \mathrm{~cm}$ bei Eingriffen in 3 Höhen. Hierauf wird die Schicht zwischen Musculus multifidus und longissimus stumpf mit dem Finger getastet und die Muskelgruppen ebenfalls stumpf getrennt bis zum Ertasten der Eintrittspunkte im medialen Bereich des Querfortsatzes der zu versorgenden Wirbel. Jetzt wird ein Sperrer mit konischer Geometrie und etwa 2,5cm Durchmesser eingesetzt, der den Aufblick auf den Entry-Punkt ermöglicht.

Im nächsten Schritt werden die Eintrittspunkte der Schrauben im Winkel zwischen Gelenkfortsatz des unteren Wirbels und Querfortsatz desselben bildwandlergesteuert gefunden und die Implantate nach Eröffnung der Kortikalis und Gewindeschnitt 
konvergierend eingebracht. Im Anschluss erfolgt das Einbringen der Längsträger in der entsprechenden Länge und die Fixierung der Platten durch Muttern, die mit einem definierten Drehmoment angezogen werden.

Letztlich dann Einlage einer Redondrainage und Wundverschluss in anatomischen Schichten.

\subsection{Statistische Methoden}

Neben der rein deskriptiven Beschreibung der erhaltenen Daten durch Häufigkeitstabellen kamen der chi²-Test (Vierfelder-Test) und der Mann-Whitney-UTest zur Darstellung der Einflussnahme einer Variable auf die zu untersuchende Variable bei unverbundenen Stichproben zur Anwendung. Desweiteren wurde dies durch Logistische Regression ermittelt.

Das Signifikanzniveau wurde für alle durgeführten statistischen Tests auf $p \leq 0,05$ festgelegt. Für die Interpretation der erhaltenen $\mathrm{p}$-Werte bedeutet dies, dass man bei einem Wert $p \leq 0,05$ mit einer Sicherheit von 95\% angeben kann, dass sich die untersuchten Gruppen unterscheiden.

\subsubsection{Chi²-Test}

Mit Hilfe von Chi²-Tests werden zwei nominal- oder ordinalskalierte Merkmale anhand von beobachteten Häufigkeiten ihrer Merkmalsausprägungen analysiert. Dabei wird untersucht, ob zwei unabhängige Merkmale assoziiert sind. 
Material und Methoden

\subsubsection{Mann-Whitney-U-Test}

Der Mann-Whitney-U-Test ist ein Test für den Vergleich zweier Stichproben, bei denen eine oder beide der zu vergleichenden Variablen aus einer Ordinalskala bestehen. Geprüft werden soll, ob eine der beiden zugehörigen Grundgesamtheiten nicht nur zufällig die größeren Elemente besitzt.

\subsubsection{Logistische Regression}

Unter logistischer Regression versteht man ein Verfahren zur, meist multivarianten, Analyse diskreter abhängiger Variablen. 


\section{Ergebnisse}

Die Ergebnisauswertung der vorliegenden Studie umfasst die radiologischen Aufnahmen von 101 Patienten sowie die ausgefüllten Fragebögen von 99 Patienten.

\subsection{Klinische Ergebnisse}

An der vorliegenden Studie beteiligten sich 68 weibliche und 52 männliche Patienten. 35 von diesen hatten ein Lebensalter von weniger als 60Jahren. Die restlichen Patienten (85) waren älter als 60Jahre.

\subsubsection{Bodymass-Index}

$18,75 \%$ der Patienten waren normalgewichtig, 39,6\% übergewichtig und $41,7 \%$ der Patienten sind als adipös, in der Adipositasgraduierung I bis III, einzustufen.

\begin{tabular}{|c|c|c|}
\hline BMI-Wert & BMI-Auswertung & Anzahl Patienten \\
\hline $18,5-24,9 \mathrm{~kg} / \mathrm{m}^{2}$ & Normalgewicht & 18 Patienten \\
\hline $25,0-29,9 \mathrm{~kg} / \mathrm{m}^{2}$ & Übergewicht & 38 Patienten \\
\hline $30,0-34,9 \mathrm{~kg} / \mathrm{m}^{2}$ & Adipositas Grad I & 27 Patienten \\
\hline $35,0-39,9 \mathrm{~kg} / \mathrm{m}^{2}$ & Adipositas Grad II & 12 Patienten \\
\hline $40,0->\mathrm{kg} / \mathrm{m}^{2}$ & Adipositas Grad III & 1 Patient \\
\hline
\end{tabular}

Tabelle 3: Body-Mass-Index 


\subsubsection{Rate an Voroperationen}

54 Patienten wurden bereits im Vorfeld der Fusionsoperation an der Lendenwirbelsäule operiert. Diese Operationen lagen zum Teil viele Jahre zurück. Für 42 Patienten war die stattgehabte Wirbelsäulenoperation die erste Operation in diesem Rahmen. 2 Patienten konnten aufgrund von Kommunikationsproblemen keine Angaben zu erfolgten Operationen machen.

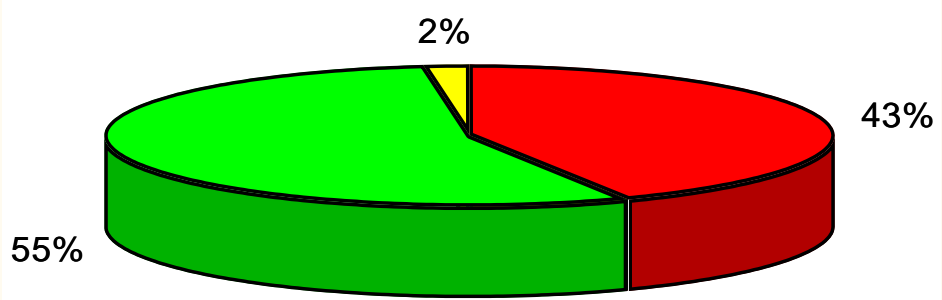

$\square$ Voroperation

$\square$ Keine Voroperation

$\square$ keine Angabe

Voroperationen

Abbildung15: Häufigkeit Voroperationen

\subsubsection{Zugangstechnik}

Bei 51 Patienten wurde der offene Operationszugang gewählt. Bei 45 Patienten wurde das Operationsgebiet durch einen transmuskulären Zugangsweg eröffnet und 2 Patienten erhielten eine Kombination aus offenem und transmuskulärem Zugang. Alle OP-Narben waren nach 12 Monaten reizlos und gut verheilt. Keiner der untersuchten Patienten gab Beschwerden im Bereich der Narbe an. 


\subsubsection{Entwicklung des Schmerzmittelbedarfs postoperativ}

Bei der Befragung bezüglich ihres Schmerzmittelbedarfs im Vergleich zur präoperativen Situation gaben $36 \%$ der Patienten an, keine Analgetika mehr zu benötigen. 37\% der Befragten berichteten, weniger schmerzstillende Medikamente einzunehmen. Bei $16 \%$ der Patienten kam es durch die Operation zu keiner Veränderung der eingenommenen Analgetikamenge. 11\% der Patienten beklagten, dass sie 12 Monate postoperativ mehr bzw. höhere Analgetikadosen im Vergleich zu präoperativ einnehmen müssten.

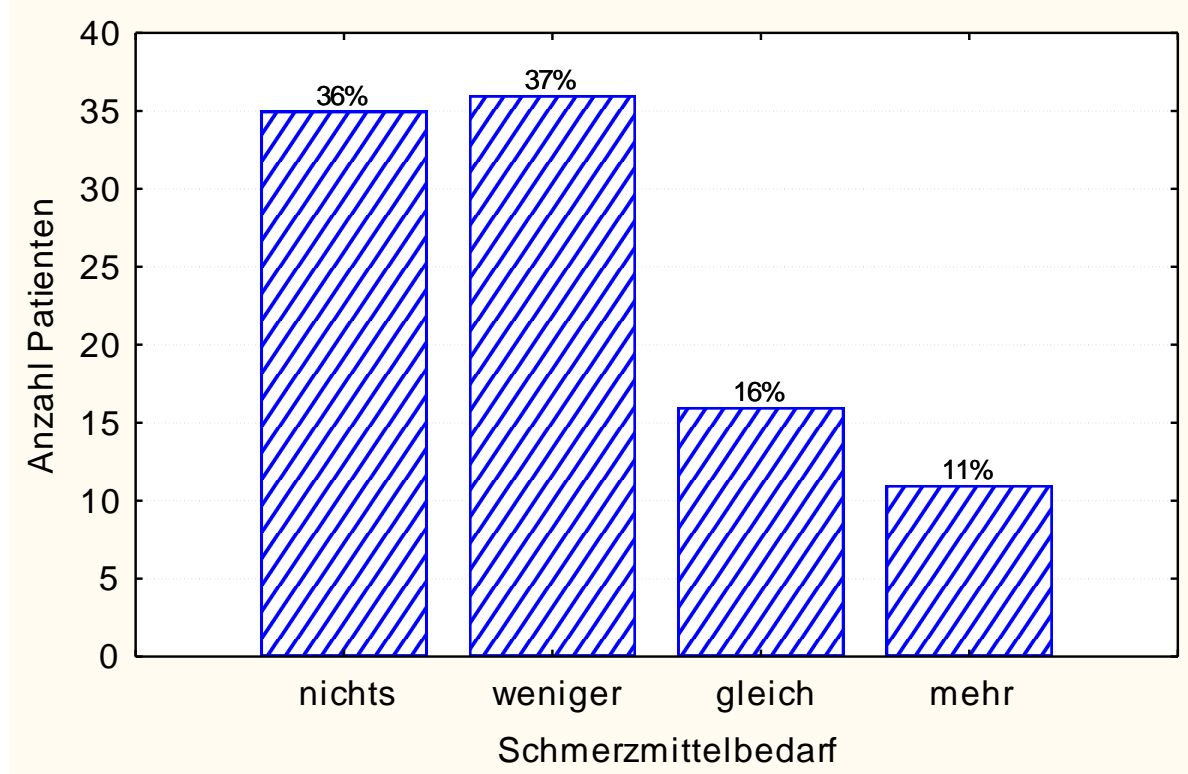

Abbildung 16: Vergleich postoperativer Schmerzmittelbedarf zu präoperativem Schmerzmittelbedarf 
Ergebnisse

In der Differenzierung des Schmerzmittelbedarfs im Vergleich zwischen den beiden Verfahren der 1. rein dynamischen Fusion und 2. der Hybridversorgung ergibt sich folgende Verteilung:

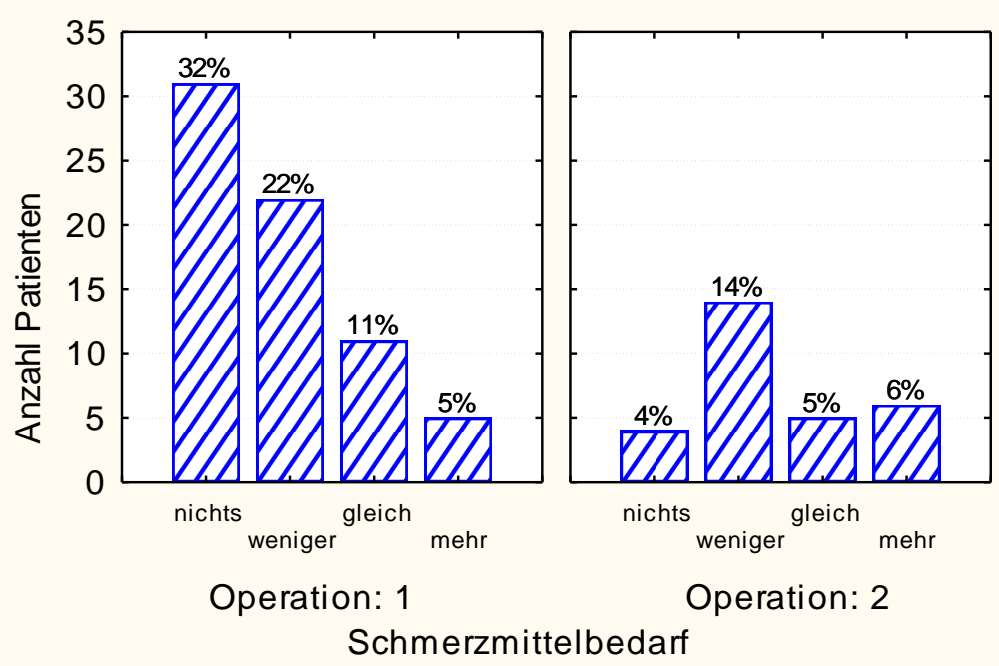

Abbildung 17: Vergleich des postoperativen

Schmerzmittelbedarfs nach Operationsumfang

\subsubsection{Nikotinkonsum}

Auf die Frage nach Nikotinkonsum gaben 70 Patienten an, Nichtraucher zu sein. 28 der befragten Patienten gaben an, zum Untersuchungszeitpunkt regelmäßig zu rauchen. 
Ergebnisse

3.1.6 Auswertung des Schmerzempfindens nach VAS

\subsubsection{Präoperatives Schmerzniveau}

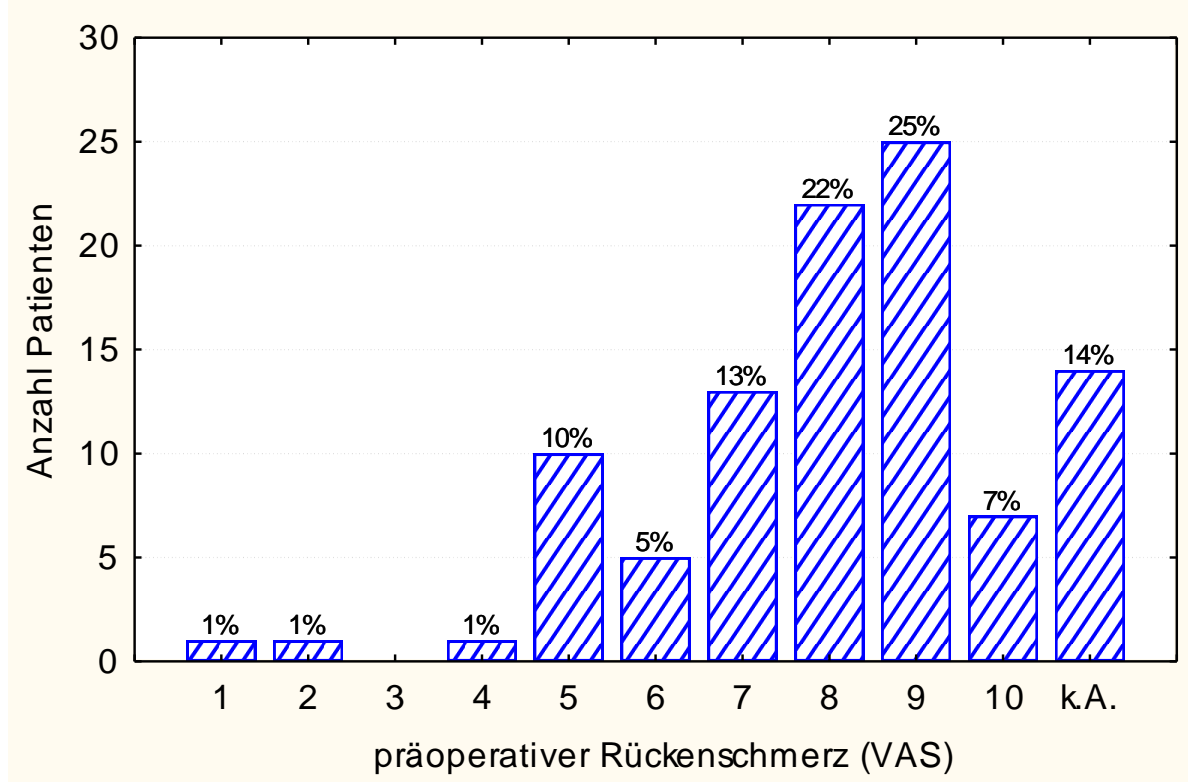

Abbildung 18: präoperativer Rückenschmerz

$13 \%$ der befragten Patienten schätzen ihren präoperativen Rückenschmerz retrospektiv mit Hilfe der Visuellen Analogskala bei einer Stärke von 0-5 (leichtemittelstarke Schmerzen) ein. $72 \%$ bewerten ihren präoperativen Schmerz mit einer Stärke von 6-10 (starke-stärkste Schmerzen). 14\% der Patienten können zu dieser Frage keine Angabe machen, da sie sich nicht mehr an den präoperativen Schmerz erinnern können. 
Ergebnisse

3.1.6.2 Postoperatives Schmerzniveau

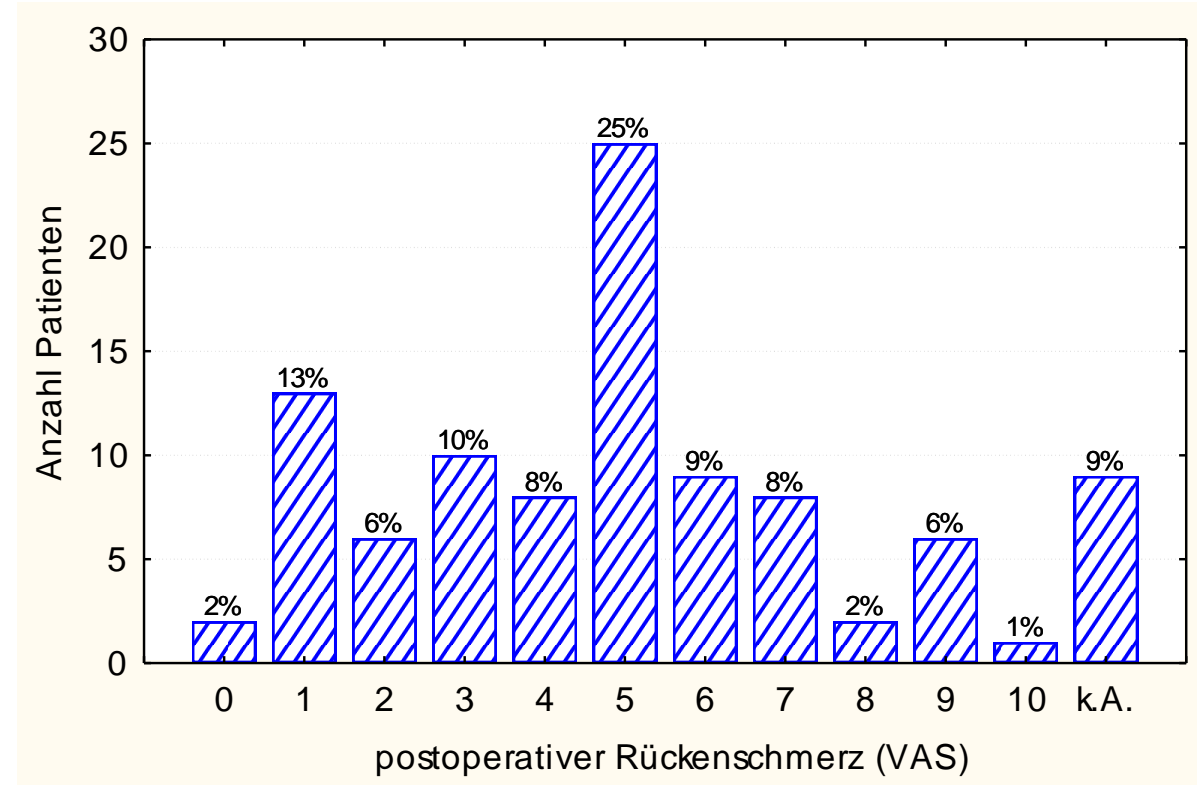

Abbildung 19: postoperativer Rückenschmerz

In der Bewertung des postoperativen Rückenschmerzes geben 64\% der Patienten leichte bis mittelstarke Schmerzen an. Starke bis stärkste Schmerzen empfinden $26 \%$ der Befragten. 
Ergebnisse

\subsubsection{Präoperative Ischialgie}

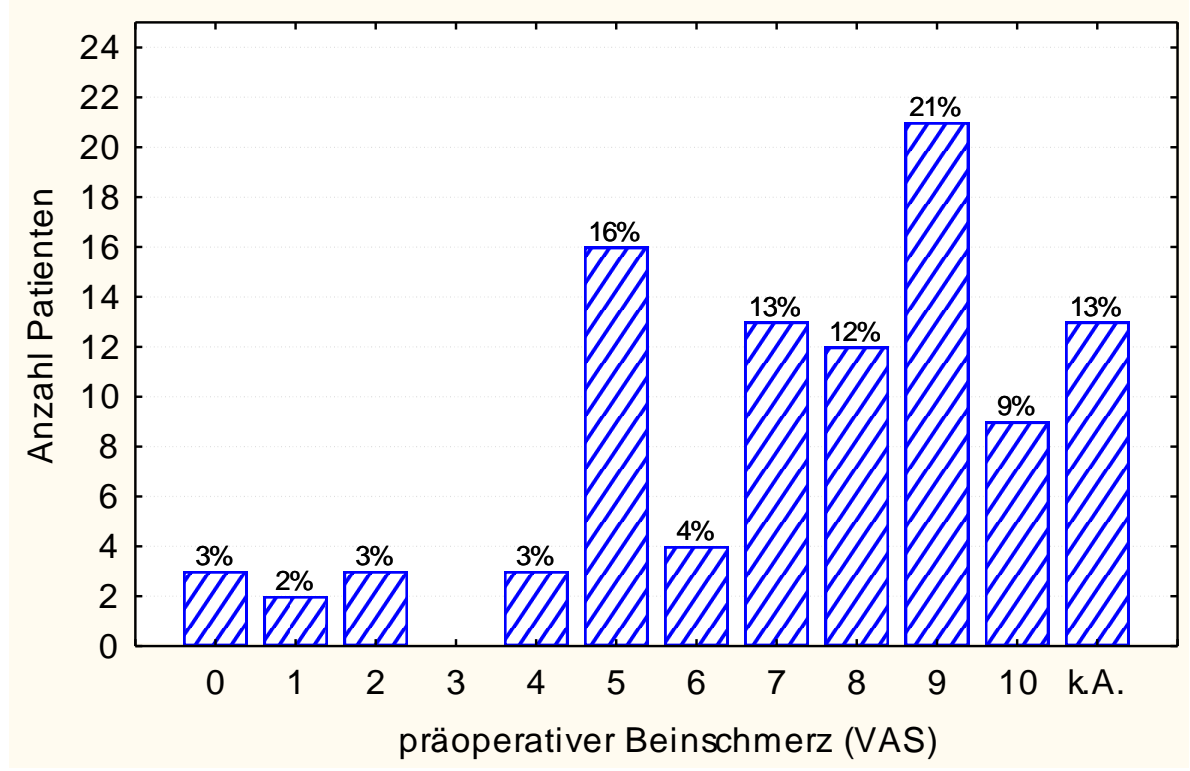

Abbildung 20: präoperative Ischialgie

$27 \%$ der Patienten gaben leichte bis mittelstarke Schmerzen an und 59\% erinnerten sich an starke bis stärkste Schmerzen.

\subsubsection{Postoperative Ischialgie}

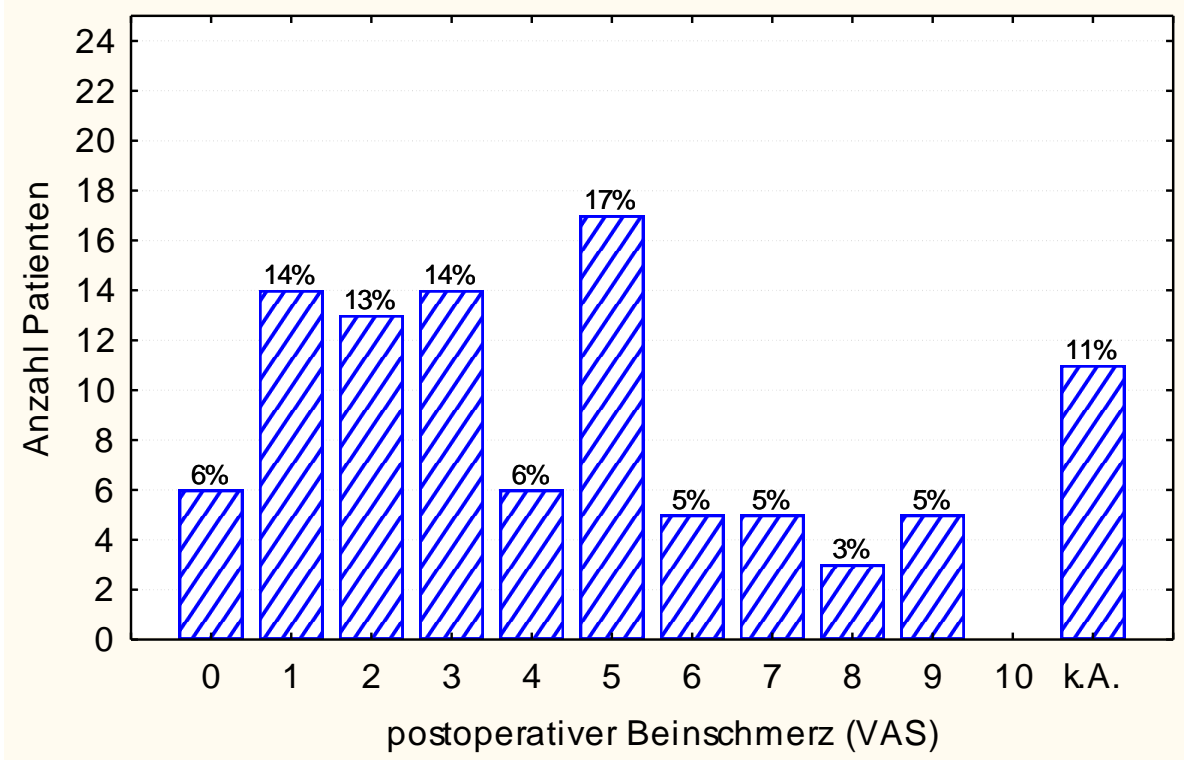

Abbildung 21: postoperative Ischialgie 
Zu Abbildung 21: Den postoperativen Beinschmerz zum Untersuchungszeitpunkt beschrieben $70 \%$ der Patienten mit leicht bis mittelstark und 18\% der Patienten weiterhin mit stark bis sehr stark.

\subsubsection{Mod. Pationnaire}

Die Auswertung der Fragen des modifizierten Pationnaire-Patientenfragebogens erfolgte mittels Häufigkeitsauszählung der gegebenen Antworten aufgetrennt in die Gruppe der Patienten mir rein dynamischer Fusion und die Gruppe der Patienten mit kombinierter dynamischer und rigider Fusion.

\subsubsection{Schmerzen bei Belastung}

Bei $50 \%$ der Patienten kann man eine postoperative Verbesserung der Schmerzsymptomatik bei Belastung beobachten. Bei $22 \%$ kam es zu einer Verschlechterung der Symptomatik. 23\% der Behandelten erzielten durch die Operation weder eine Verbesserung noch eine Verschlechterung.

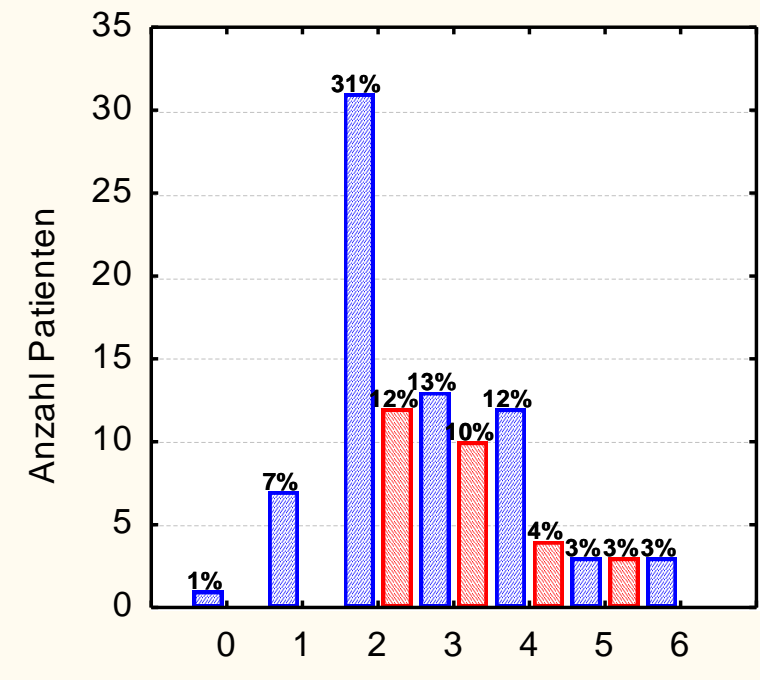

Schmerzen bei Belastung
$0=$ präoperativ nicht vorhanden $1=$ viel besser 2=besser

$3=$ gleich

4=schlechter

$5=$ viel schlechter

$6=$ keine Angabe rein dynamisch dynamisch+rigide

Abbildung 22: Schmerzen bei Belastung postoperativ im Vergleich zu präoperativ 
Ergebnisse

\subsubsection{Schmerzen in Ruhe}

$65 \%$ der Patienten gaben eine Verbesserung des Schmerzes in Ruhe während des postoperativen Verlaufs an.

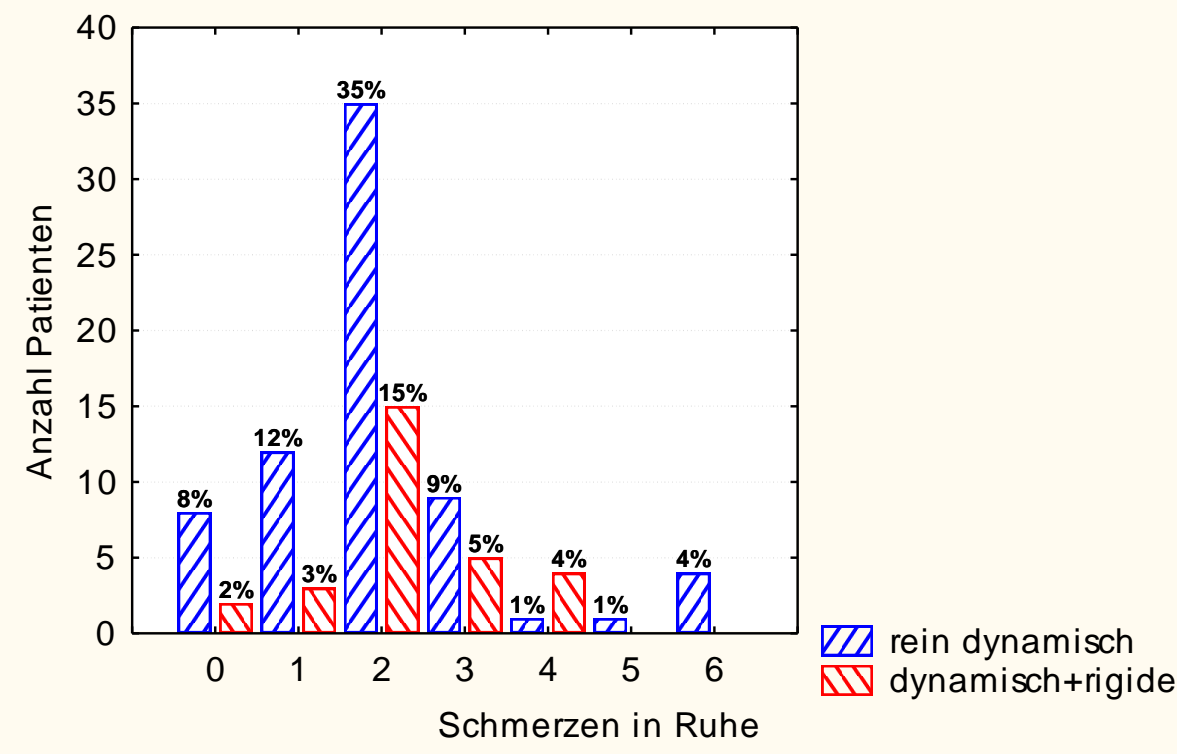

$0=$ präoperativ nicht vorhanden

$1=$ viel besser

$2=$ besser

$3=$ gleich

$4=$ schlechter

$5=$ viel schlechter

$6=$ keine Angabe

Abbildung 23: Schmerzen in Ruhe postoperativ im Vergleich zu präoperativ 
Ergebnisse

\subsubsection{Schmerzen nachts}

64\% der Patienten berichten über eine Verbesserung der nächtlichen Schmerzsymptomatik.
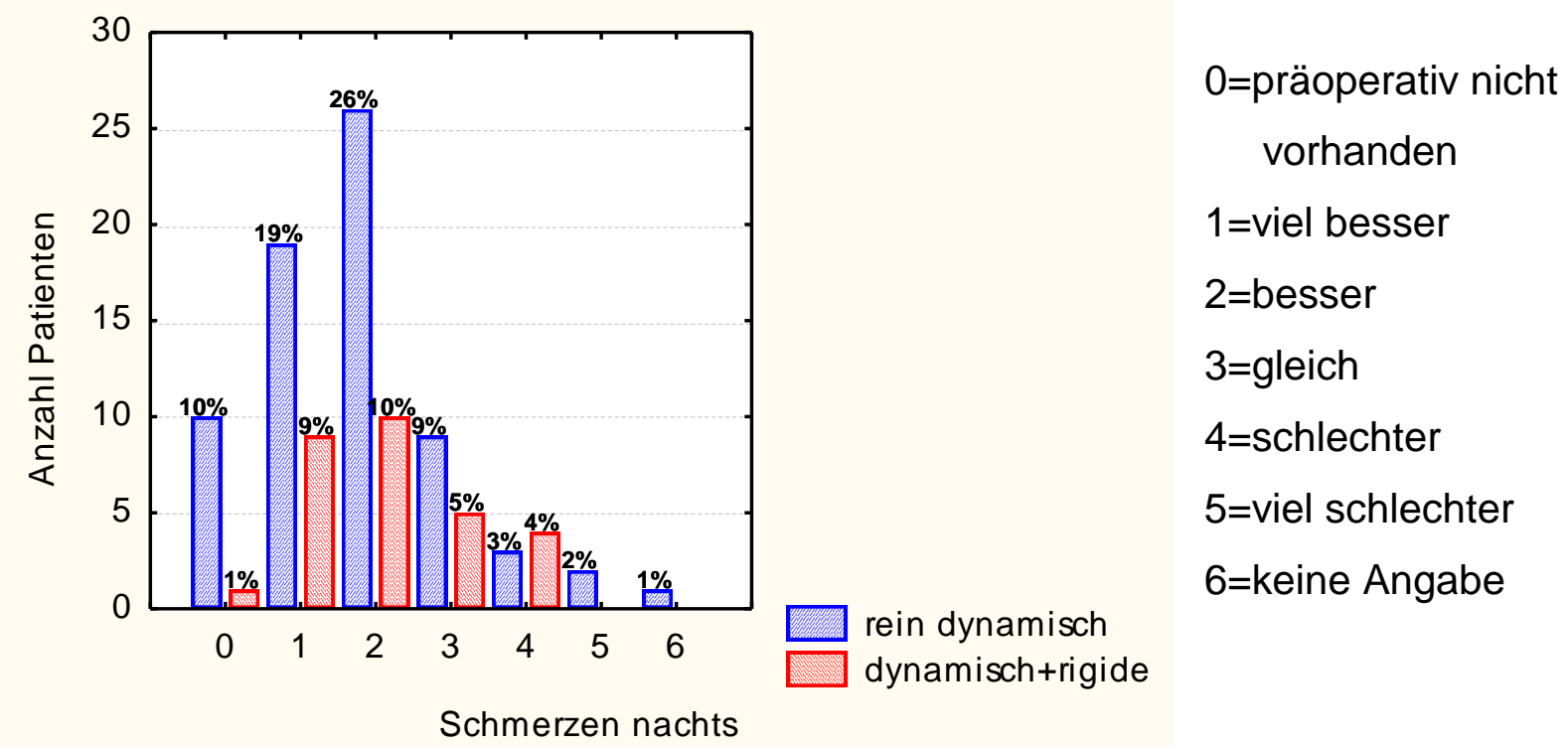

Abbildung 24: Schmerzen nachts postoperativ im Vergleich zu präoperativ 
Ergebnisse

\subsubsection{Brenndysästhesien}

49\% der befragten Patienten gaben an, präoperativ kein Brennen verspürt zu haben. Bei weiteren 30\% kam es zur Besserung der Beschwerden.
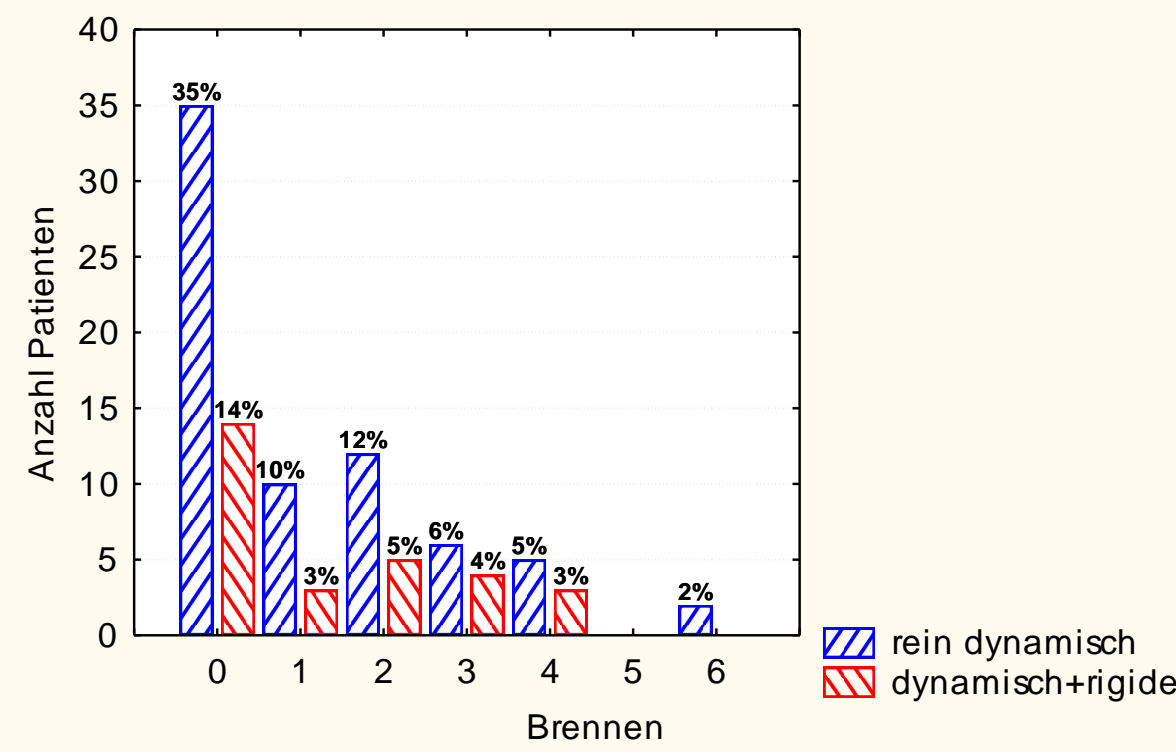

$0=$ präoperativ nicht vorhanden

$1=$ viel besser

$2=$ besser

$3=$ gleich

$4=$ schlechter

$5=$ viel schlechter

$6=$ keine Angabe

Abbildung 25: Brenndysästhesien postoperativ im Vergleich zu präoperativ 
Ergebnisse

\subsubsection{Krämpfe}

Auch hier ist eine Besserungstendenz zu sehen, auch wenn $45 \%$ der Patienten präoperativ nicht unter Krämpfen litten.

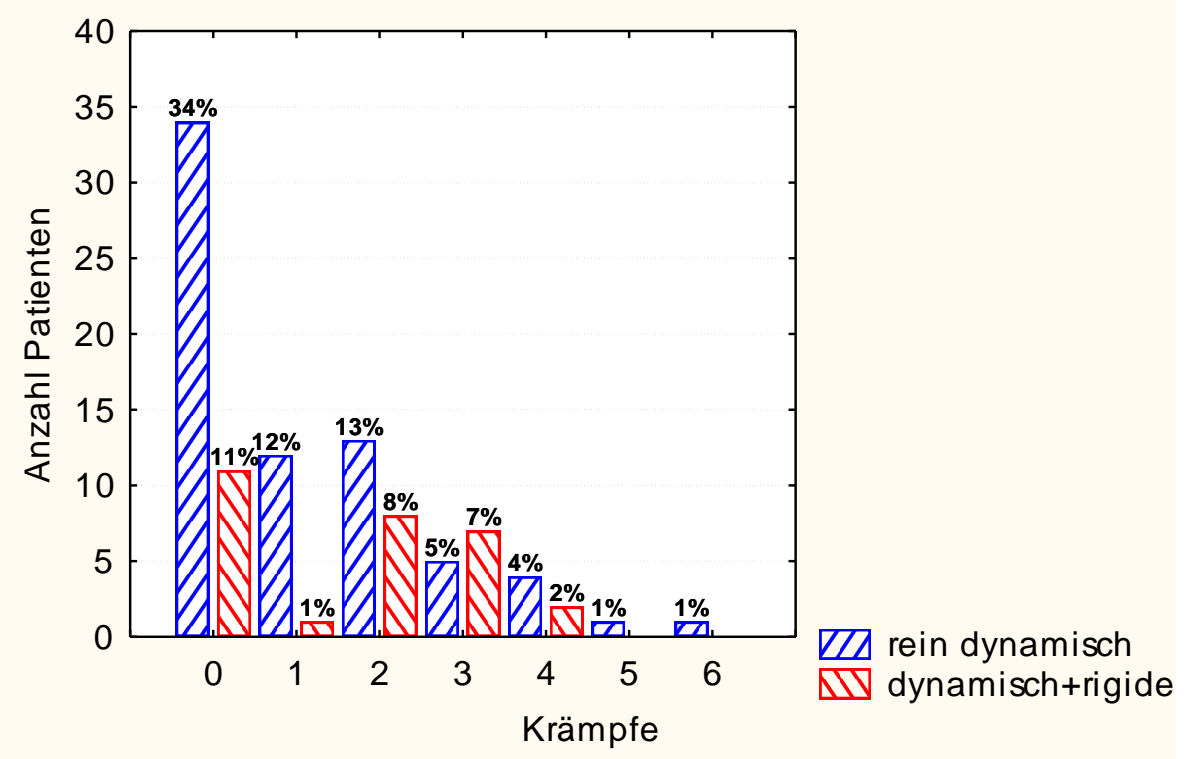

$0=$ präoperativ nicht vorhanden

$1=$ viel besser

$2=$ besser

$3=$ gleich

$4=$ schlechter

$5=$ viel schlechter

$6=$ keine Angabe

Abbildung 26: Krämpfe postoperativ im Vergleich zu präoperativ 
Ergebnisse

\subsubsection{Schwäche / Kraftlosigkeit}

42\% der Patienten geben eine weiterhin bestehende, zum Teil zunehmende Kraftlosigkeit an. 37\% hingegen berichten über eine Verbesserung der postoperativ bestandenen Kraftlosigkeit.

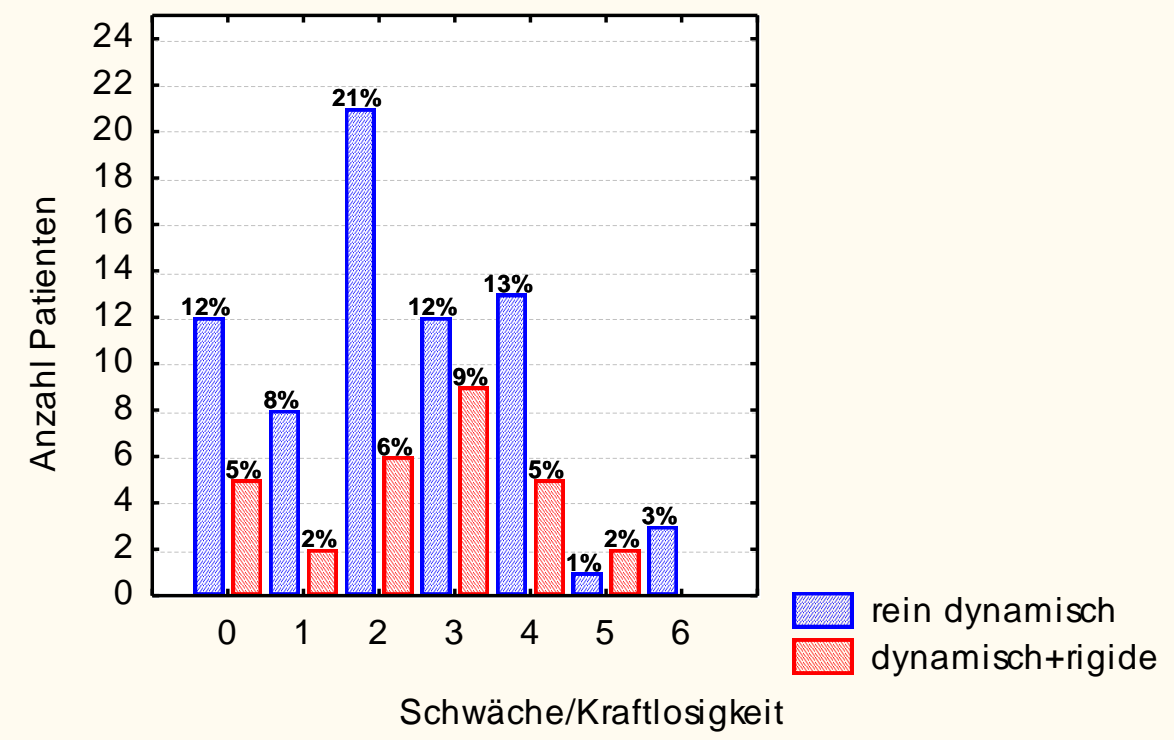

$0=$ präoperativ nicht vorhanden

$1=$ viel besser

$2=$ besser

$3=$ gleich

$4=$ schlechter

$5=$ viel schlechter

$6=$ keine Angabe

Abbildung 27: Schwäche/Kraftlosigkeit postoperativ im Vergleich zu präoperativ 
Ergebnisse

\subsubsection{Kribbelparästhesien}

$41 \%$ der nachuntersuchten Patienten berichten von einer Besserung der präoperativ bestandenen Parästhesien. Bei $20 \%$ der Befragten beobachtete man eine Verschlechterung der Beschwerden. 16\% der Befragten verspürten keine wesentliche Änderung im Verlauf des ersten postoperativen Jahres.

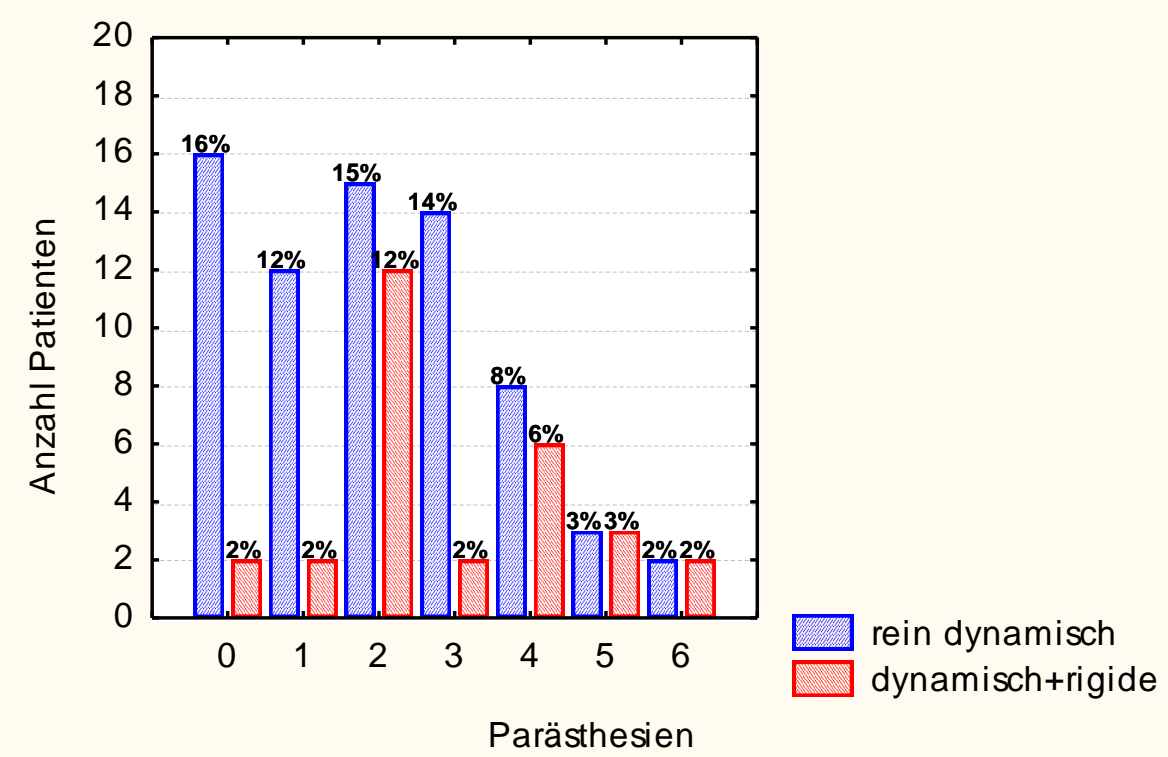

$0=$ präoperativ nicht vorhanden

$1=$ viel besser

2=besser

$3=$ gleich

$4=$ schlechter

$5=$ viel schlechter

$6=$ keine Angabe

Abbildung 28: Parästhesien postoperativ im Vergleich zu präoperativ 


\subsubsection{Allgemeine „Steifheit"}

Bei dieser Frage gab es von Seiten der Patienten häufig Verständnisschwierigkeiten, die dann im Verlauf des Anamnesegespräches geklärt werden konnten. 30\% berichteten, dass sie dieses Beschwerdebild nicht an sich entdecken konnten. 31\% konnten eine Besserung angeben. Bei $12 \%$ der Patienten verschlechterte sich die Symptomatik.

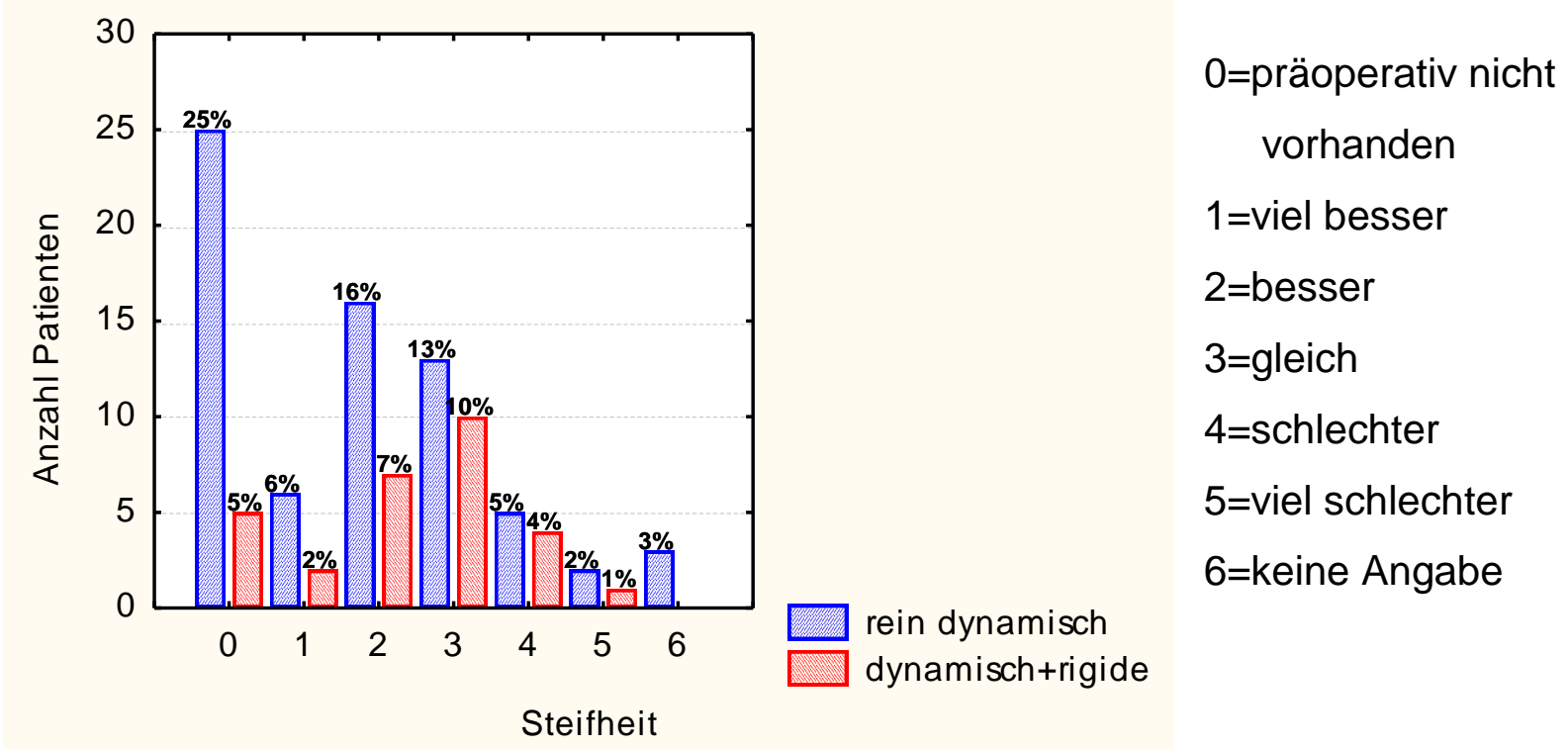

Abbildung 29: Steifheit postoperativ im Vergleich zu präoperativ 
Ergebnisse

\subsubsection{Bewegungseinschränkung}

Dieses Symptom wurde von den Patienten als sehr belastend empfunden. 46\% gaben keine Besserung an. Bei $42 \%$ der Befragten konnte eine gute Besserung erkannt werden.

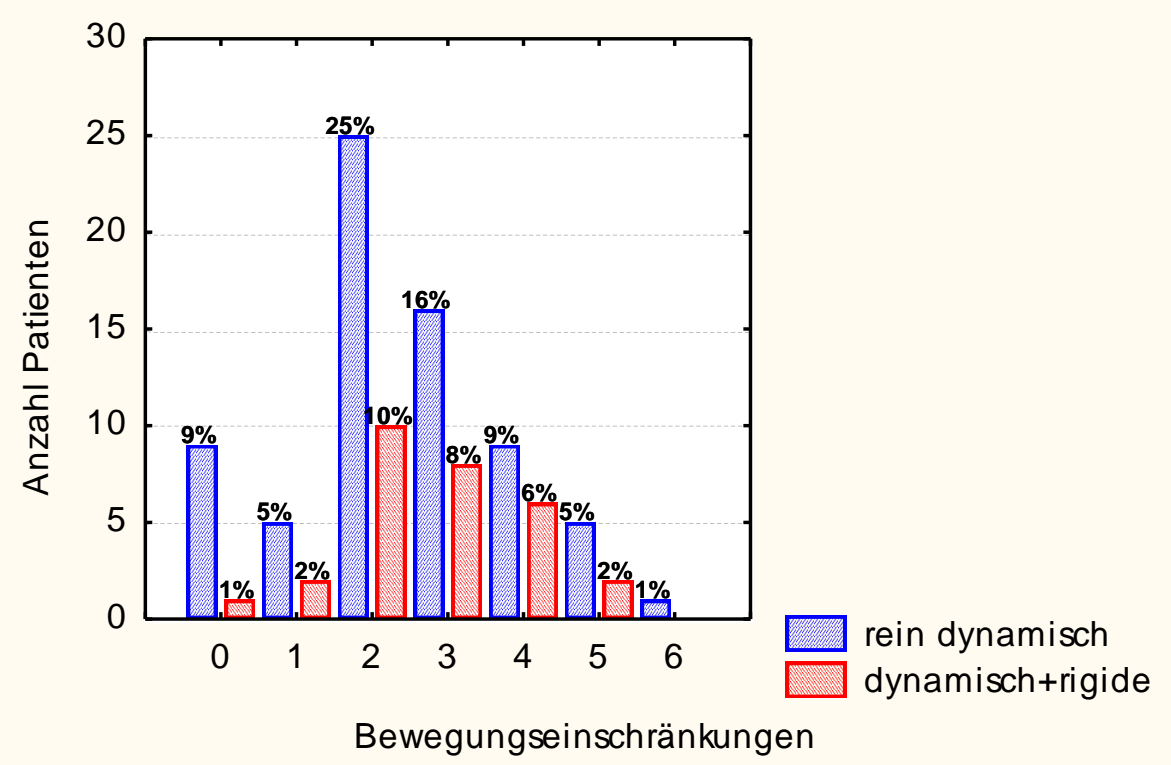

$0=$ präoperativ nicht vorhanden

$1=$ viel besser

$2=$ besser

$3=$ gleich

$4=$ schlechter

$5=$ viel schlechter

$6=$ keine Angabe

Abbildung 30: Bewegungseinschränkung postoperativ im Vergleich zu präoperativ 
Ergebnisse

\subsubsection{Schlafen}

62\% der dynamisch und kombiniert dynamisch+rigide fusionierten Patienten gaben an, dass sich ihr Schlaf postoperativ verbessert habe. Bei 10\% der Patienten kam es zu einer Verschlechterung der Schlafqualität.

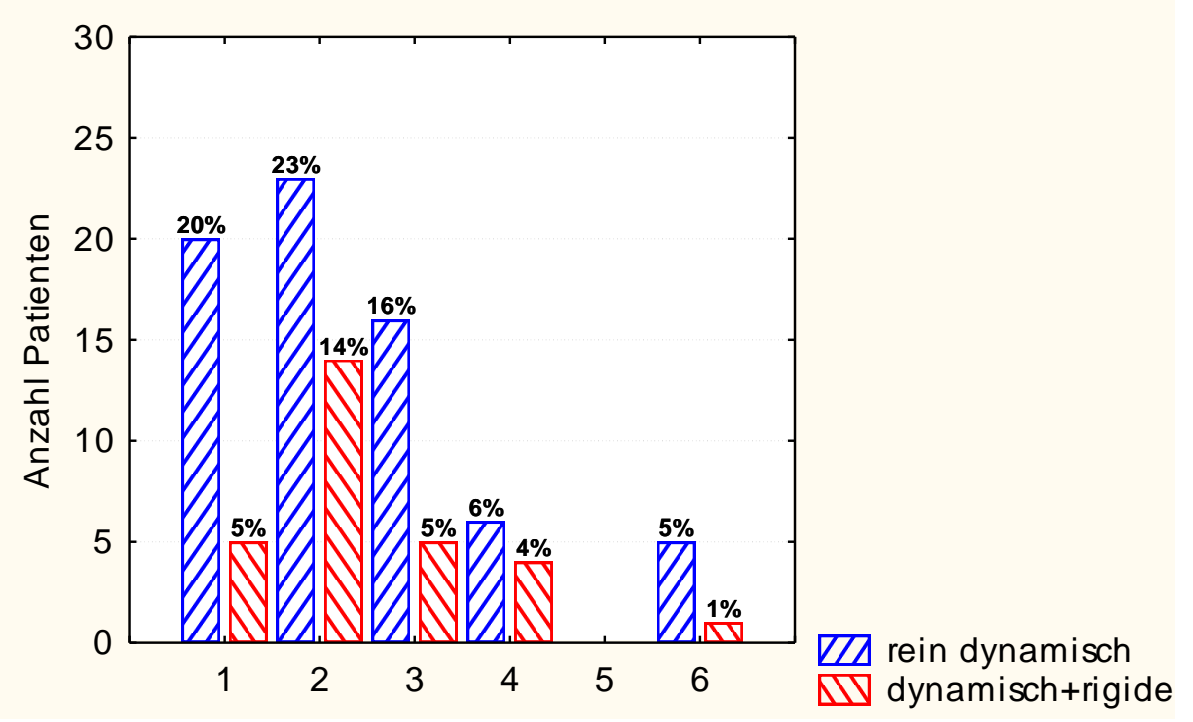

$1=$ viel besser

$2=$ besser

$3=$ gleich

$4=$ schlechter

$5=$ viel schlechter

$6=$ keine Angabe

Abbildung 31: Veränderung des Schlafs postoperativ im Vergleich zu präoperativ 
Ergebnisse

\subsubsection{Waschen/Kleiden}

$50 \%$ der untersuchten Patienten berichteten, dass innen die Aktivitäten des täglichen Lebens, wie z.B. Waschen und Ankleiden, postoperativ wieder leichter fallen.

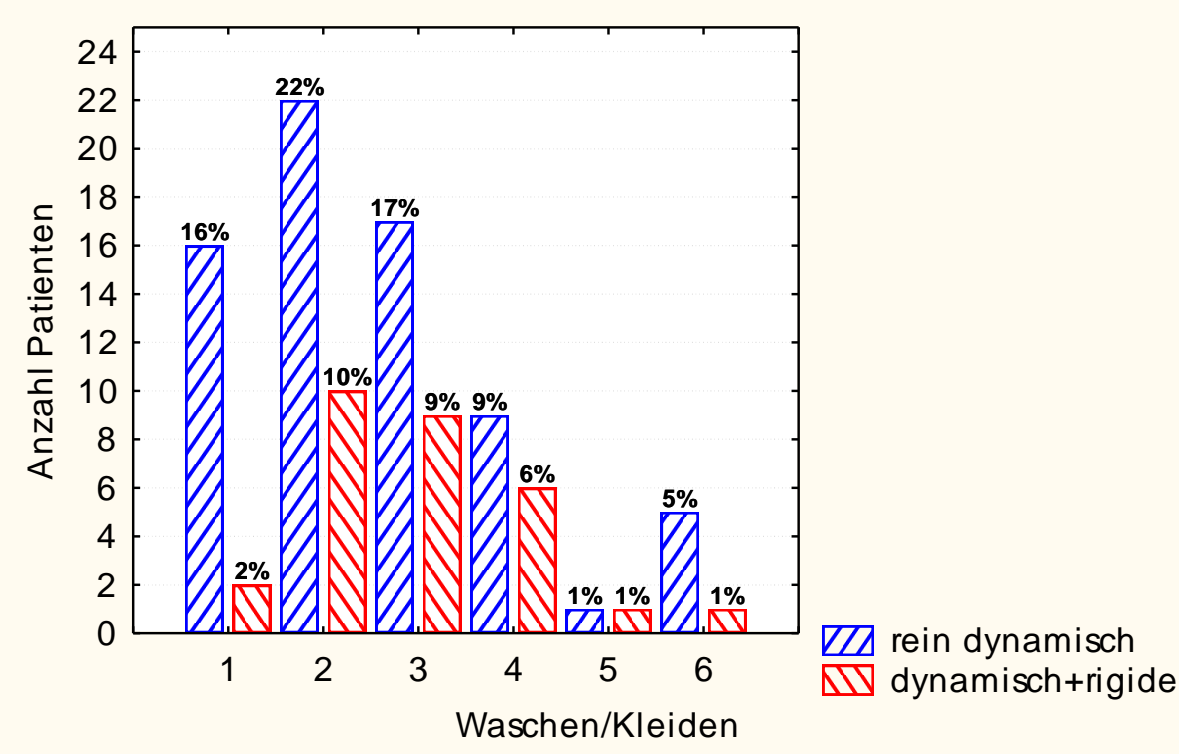

$1=$ viel besser

$2=$ besser

$3=$ gleich

$4=$ schlechter

$5=$ viel schlechter

6=keine Angabe

Abbildung 32: Veränderung beim Waschen / Kleiden postoperativ im Vergleich zu präoperativ 
Ergebnisse

\subsubsection{Sitzen}

Für 48\% der Befragten ist es postoperativ möglich wieder problemlos längere Zeit zu Sitzen. 13\% der Patienten gaben keine Verbesserung an.

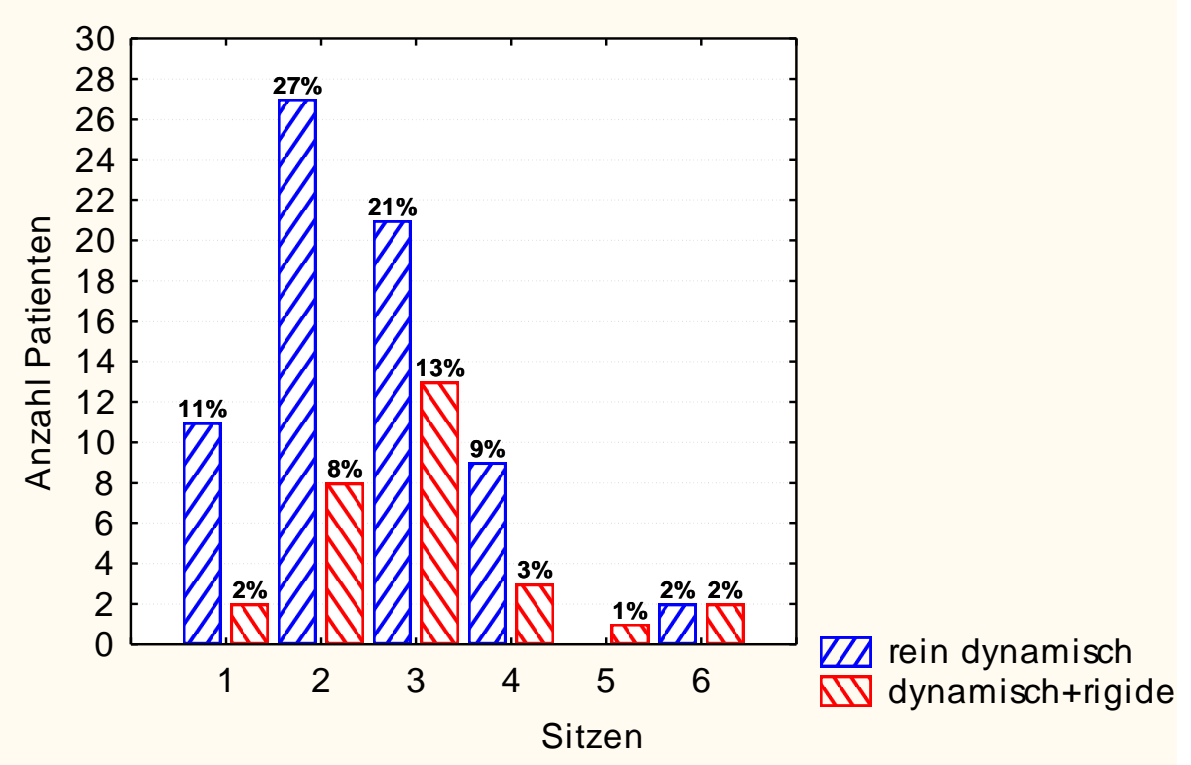

$1=$ viel besser

$2=$ besser

$3=$ gleich

$4=$ schlechter

$5=$ viel schlechter

$6=$ keine Angabe

Abbildung 33: Veränderung Sitzfähigkeit postoperativ im Vergleich zu präoperativ 
Ergebnisse

\subsubsection{Stehen}

Längeres Stehen bereitete vielen Patienten präoperativ deutliche Schwierigkeiten. Bei $37 \%$ der Patienten besserte sich dies in den ersten 12 postoperativen Monaten. $30 \%$ der Befragten gaben an, dass noch keine Besserung eingetreten sei, es sich jedoch auch nicht verschlechtert habe.

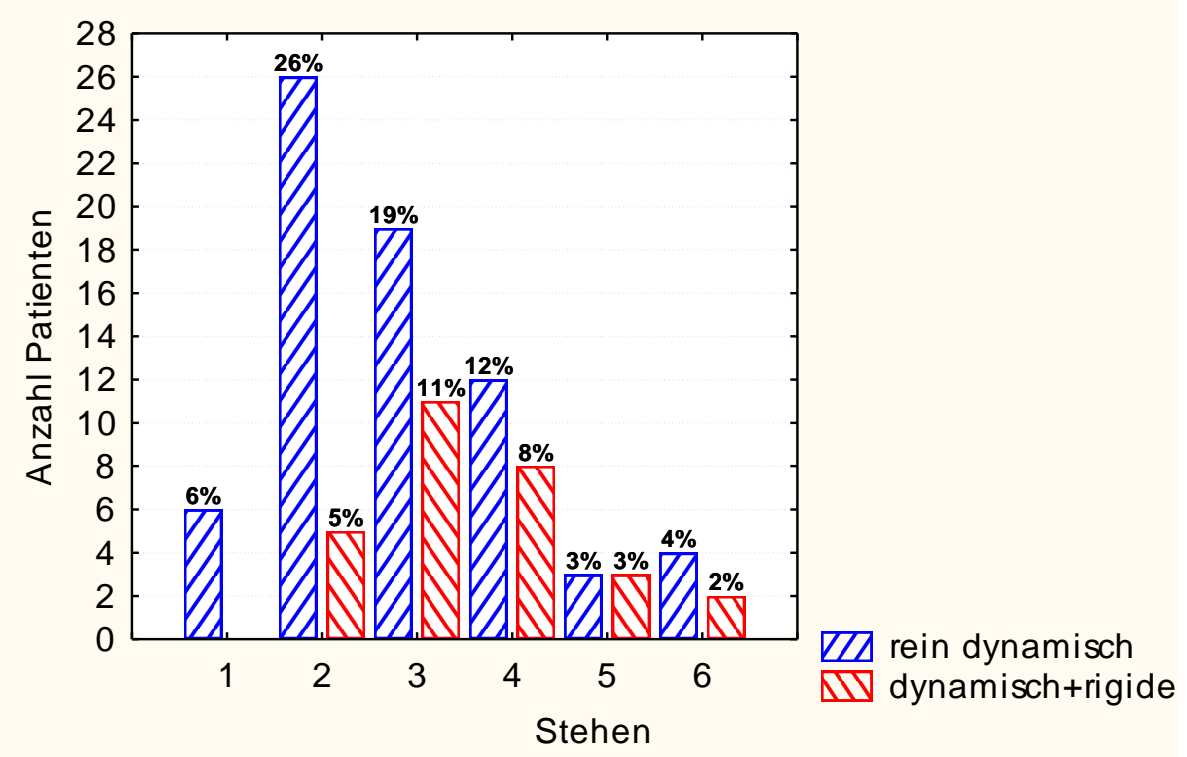

$1=$ viel besser

$2=$ besser

$3=$ gleich

$4=$ schlechter

$5=$ viel schlechter

$6=$ keine Angabe

Abbildung 34: Veränderung Stehfähigkeit postoperativ im Vergleich zu präoperativ 


\subsubsection{Gehen}

Die Frage nach der Möglichkeit, längere Strecken zu gehen, bewerteten 48\% als gebessert. Bei 17\% der Patienten änderte sich nichts an der Gehstrecke und bei 29\% der Patienten verschlechterte sich die Gehfähigkeit.

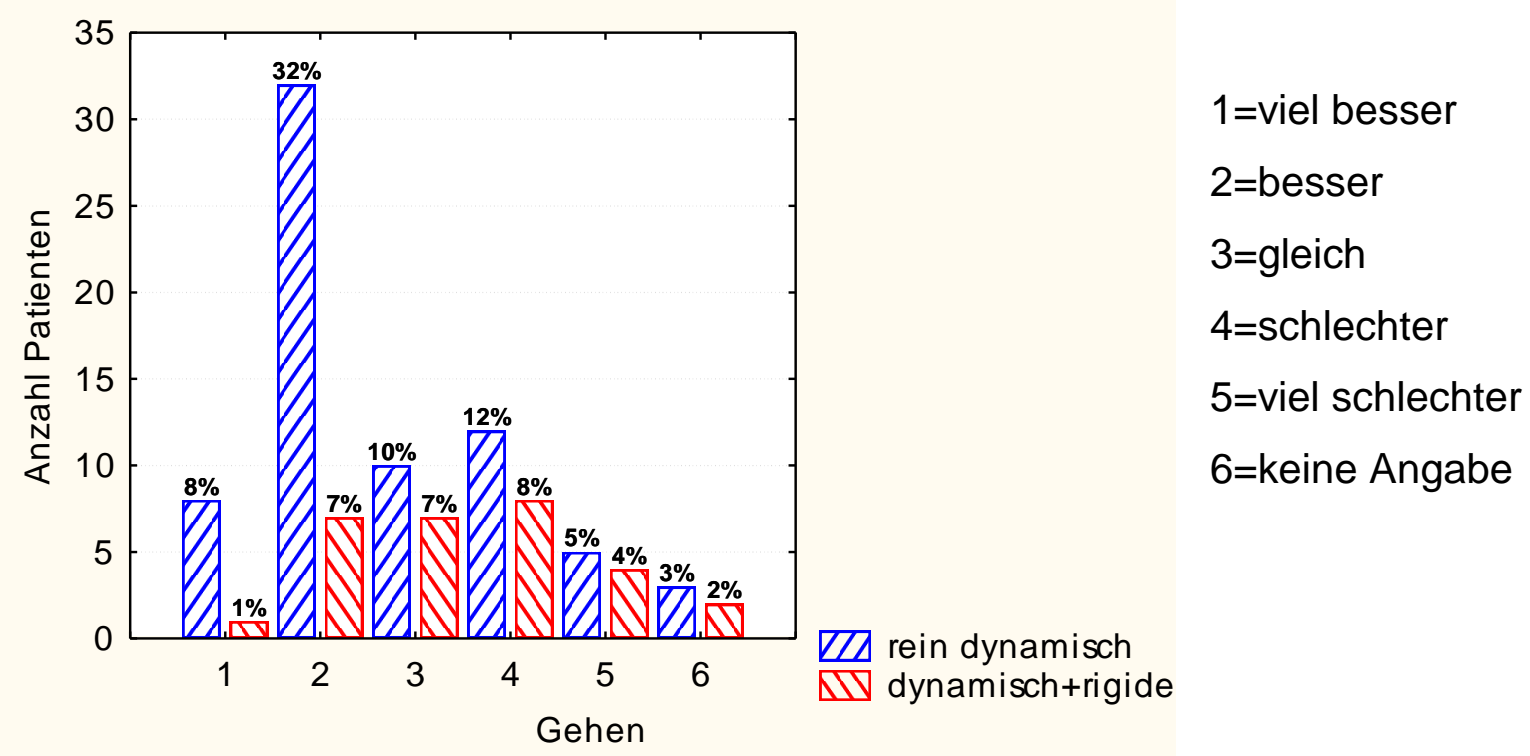

Abbildung 35: Veränderung Gehfähigkeit postoperativ im Vergleich zu präoperativ

\subsubsection{Soziales Leben}

$40 \%$ der nachuntersuchten Patienten gaben an, dass sich ihr Interesse am sozialen Leben, d.h. Freunde besuchen, Ausgehen, wieder gesteigert habe. Bei 25\% der Patienten ließ sich keine Besserung erkennen. Diese reduzierten ihre diesbezüglichen Aktivitäten im postoperativen Verlauf. 


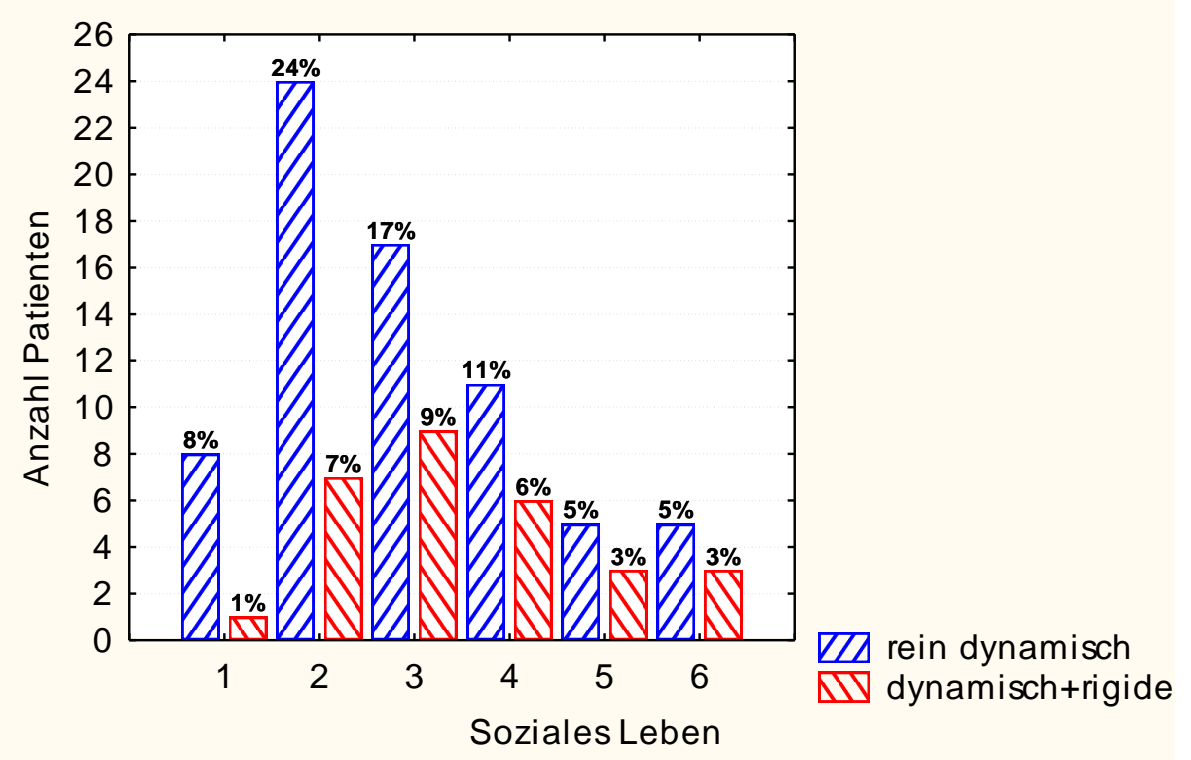

$1=$ viel besser

$2=$ besser

$3=$ gleich

4=schlechter

$5=$ viel schlechter

$6=$ keine Angabe

Abbildung 36: Veränderung der Teilnahme am Sozialen Leben

postoperativ im Vergleich zu präoperativ

\subsubsection{Zufriedenheit mit jetziger Situation}

Im Rahmen der klinischen Nachuntersuchung wurden die Patienten gebeten, anzugeben, inwieweit sie mit der postoperativen Situation, d.h. mit ihrer Lebensqualität zufrieden sind. Hierbei konnte man beobachten, dass $48 \%$ der Befragten zufrieden bis sehr zufrieden mit ihrer Lebenssituation sind. $8 \%$ der Patienten gaben jedoch an, sehr unzufrieden zu sein. 


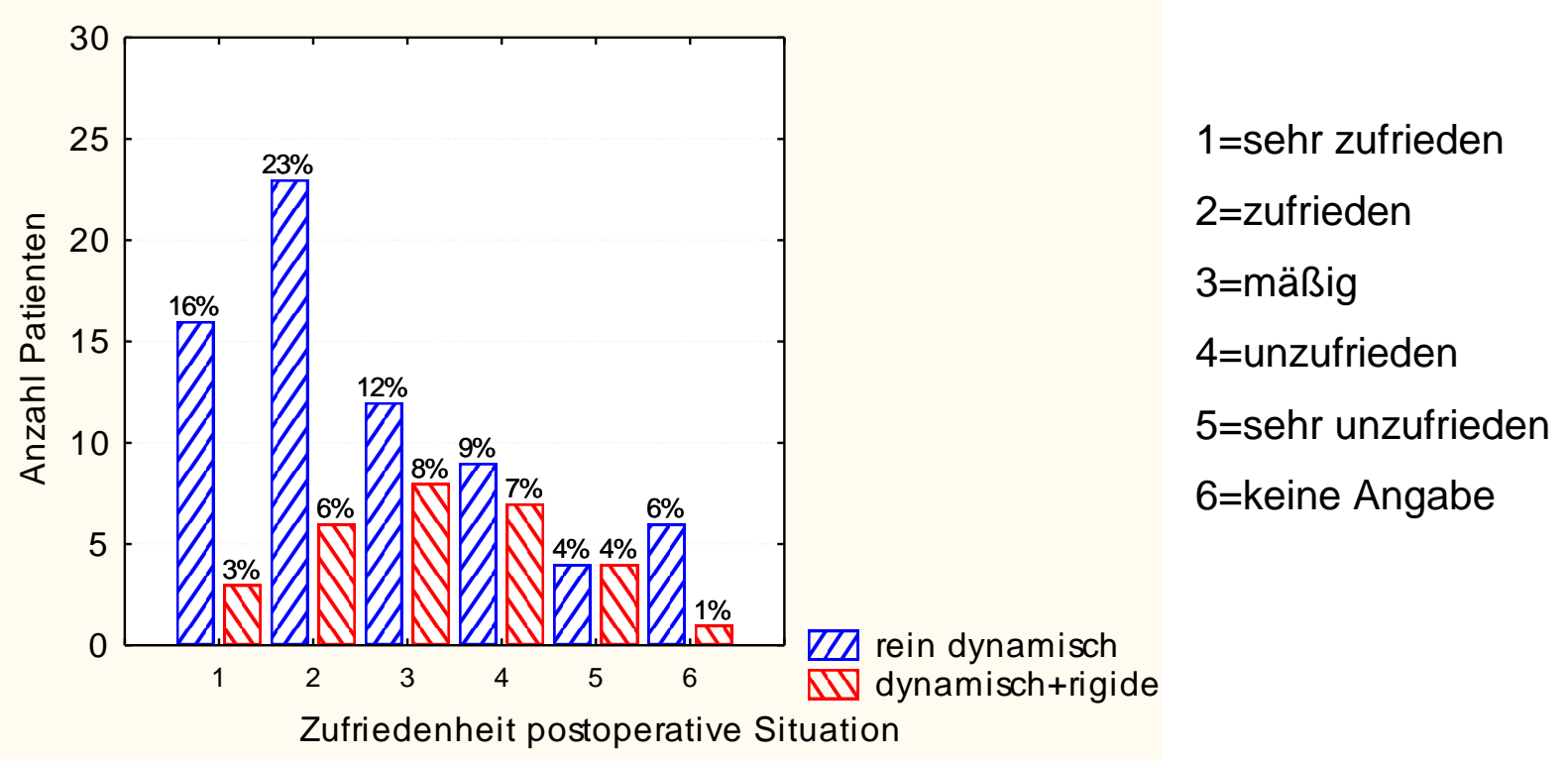

Abbildung 37: Zufrieden mit postoperativer Situation

Nachdem die Patienten gebeten wurden, ihre Zufriedenheit mit der postoperativen Situation anzugeben, wurden sie bezüglich einer erneuten OP-Motivation befragt. 82\% der Patienten würden nochmals die Entscheidung für die Fusionsoperation treffen, so wie auch schon 1 Jahr zuvor. 10\% der Patienten würden die Operation bei nochmaliger Entscheidungsmöglichkeit ablehnen. Zumeist aus Angst (Informationen aus den Befragungssituationen), aber auch aufgrund von zwischenzeitlich aufgetretenen Comorbiditäten. 8\% der Patienten verweigerten die Aussage, da dies zum Zeitpunkt der Nachuntersuchung nicht relevant sei. 


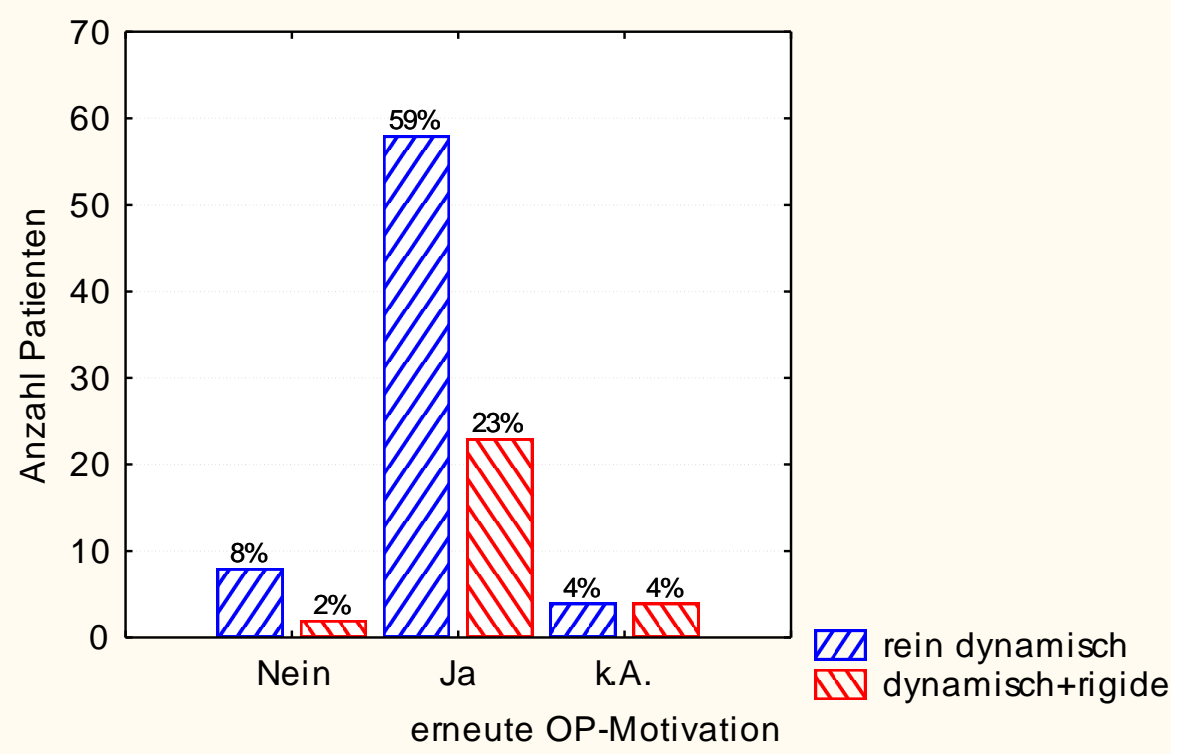

Abbildung 38: erneute Op-Motivation

\subsubsection{Oswestry-Score-Auswertung}

Die Auswertung der Fragen des Oswestry-Scores erfolgt anhand der erreichten maximalen Punktzahl bzw. der daraus resultierenden Prozentwerte. Die Patienten aus der Gruppe der rein dynamischen Fusion schätzten ihre Beeinträchtigung mit bestenfalls $0 \%$ bis $76 \%$, bei einem Median von $36 \%$, ein. Die Patienten der kombiniert fusionierten Gruppe erhoben durch die Beantwortung der Fragen Prozentwerte von $16 \%$ bis $84 \%$, bei einem Median von $50 \%$. Zur Erklärung der Prozentwerte siehe folgende Tabelle.

\begin{tabular}{|l|l|}
\hline \multicolumn{1}{|c|}{ Prozentwerte } & \multicolumn{1}{|c|}{ Ausprägung der Beeinträchtigung } \\
\hline $0-20 \%$ & minimale Behinderung \\
\hline $20-40 \%$ & mäßige Behinderung \\
\hline $40-60 \%$ & starke Behinderung \\
\hline $60-80 \%$ & invalidisierend \\
\hline $80-100 \%$ & bettlägerig \\
\hline
\end{tabular}

Tabelle 4: Auswertung Oswestry-Score 


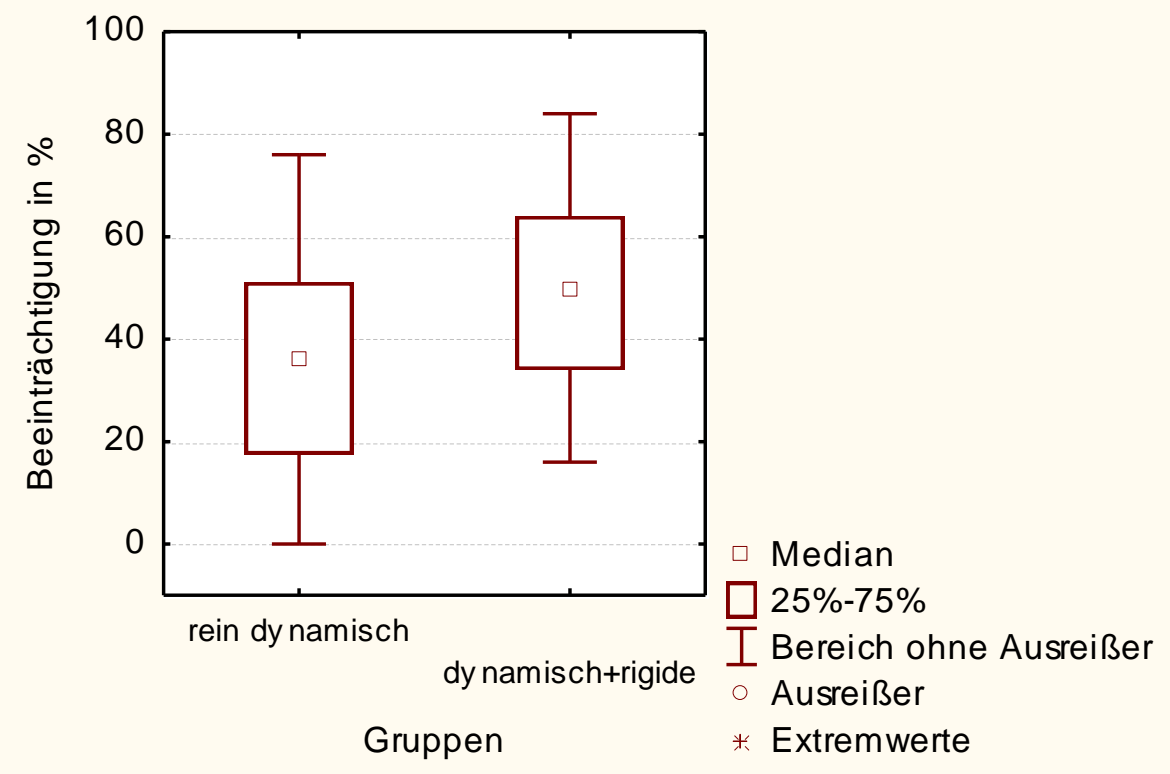

Abbildung 39: Verteilung Oswestry Score in Bezug auf OP-Umfang

Insgesamt sehen sich $50 \%$ der Patienten als minimal bis mäßig behindert. Weitere $31 \%$ empfinden ihre Einschränkungen als stark behindert und 15\% fühlen sich dadurch gar invalidisiert. Bei $2 \%$ der auszuwertenden Fragebögen erhielt man das Ergebnis „Bettlägerigkeit“

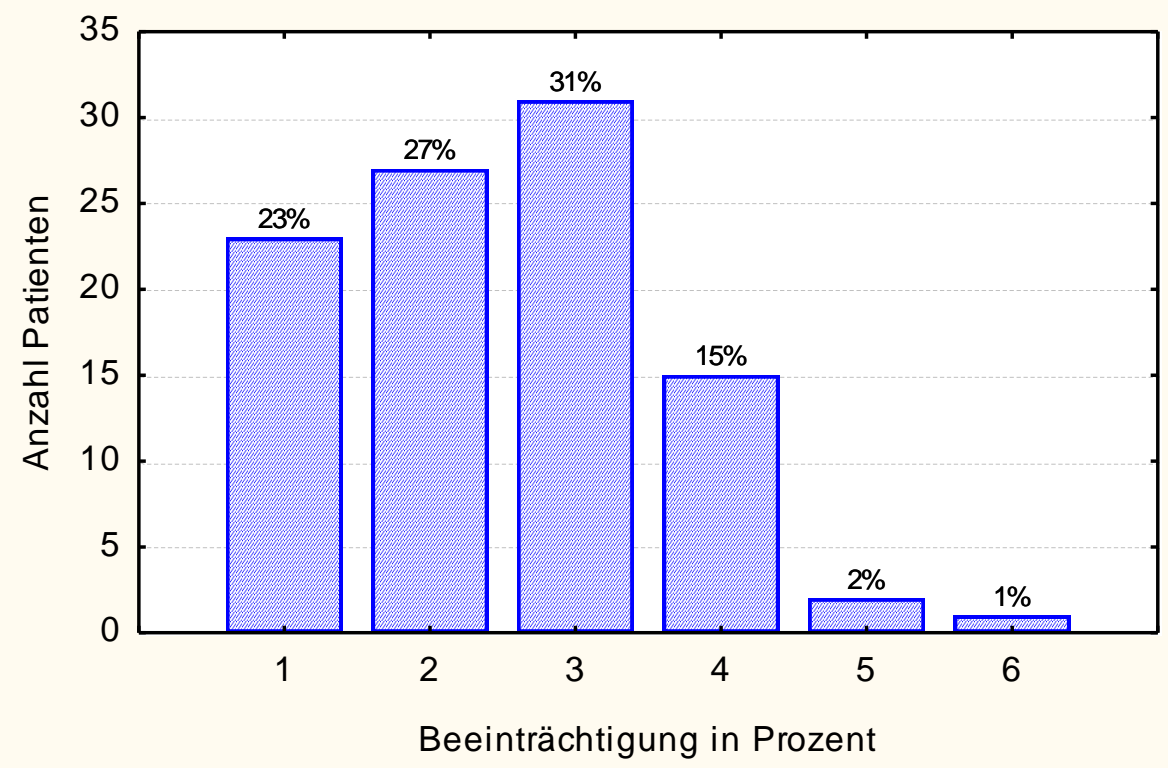

$\begin{array}{lrr}\text { 1: } & 0-20 \% & \begin{array}{r}\text { Minimale } \\ \text { Behinderung }\end{array} \\ \text { 2: } & 20-40 \% & \begin{array}{r}\text { Mäßige } \\ \text { Behinderung }\end{array} \\ \text { 3: } & 40-60 \% & \begin{array}{r}\text { Starke } \\ \text { Behinderung }\end{array} \\ \text { 4: } & 60-80 \% & \text { invalidisierend } \\ \text { 5: } & 80-100 \% & \text { bettlägerig }\end{array}$

Abbildung 40: Häufigkeitsverteilung der Beeinträchtigung Oswestry Score 


\subsection{Radiologische Ergebnisse}

Insgesamt wurden bei 99 Patienten 462 Schrauben, im Verhältnis 202 dynamische Schrauben zu 260 rigiden Schrauben, eingebracht.

\subsubsection{Schraubenlockerungen}

Bei der Auswertung der 12 Monate postoperativ angefertigten Computertomographie-Aufnahmen konnte bei 20 Patienten eine Schraubenlockerung diagnostiziert werden. Bei 6 Patienten kam es zur Lockerung der dynamischen Schrauben, bei 12 Patienten der rigiden und bei 2 Patienten lockerten sich sowohl dynamische als auch rigide Schrauben. Die Anzahl der gelockerten Schrauben beläuft sich auf 34, wovon 11 Schrauben dynamischen Charakter und 23 Schrauben rigiden Charakter aufweisen. Somit erhält man eine Lockerungsrate von $~ 5,4 \%$ bei dynamischen Schrauben und eine Lockerungsrate von $\sim 8,8 \%$ bei rigiden Schrauben.

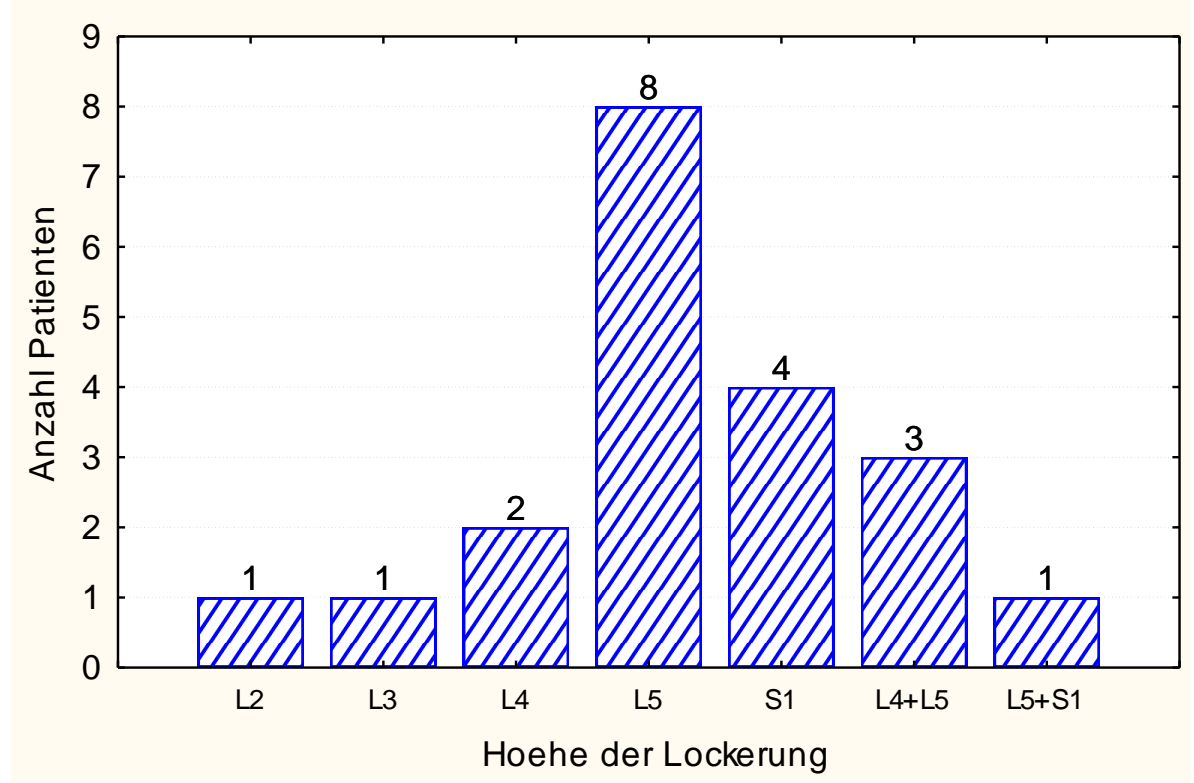

Abbildung 41: Häufigkeitsverteilung Höhe der Schraubenlockerung 


\subsubsection{Schraubenbrüche}

Bei 2 Patienten kam es im Verlauf des ersten postoperativen Jahres zu einem Schraubenbruch jeweils einer rigiden Schraube. Hierdurch ergibt sich eine Bruchrate von $\sim 0,8 \%$.

\begin{tabular}{|c|c|}
\hline Höhe Schraubenbruch & Anzahl Patienten \\
\hline LWK 5 & 1 Patient \\
\hline SWK 1 & 1 Patient \\
\hline
\end{tabular}

Tabelle 5: Häufigkeitsverteilung Höhe der Schraubenbrüche

\subsubsection{Fusionsrate}

51 Patienten (50,5\%) wiesen in den 1-Jahres-Kontrollaufnahmen die radiologischen Kriterien einer knöchernen Fusion und somit Verlust des dynamischen Bewegungsaspektes auf.

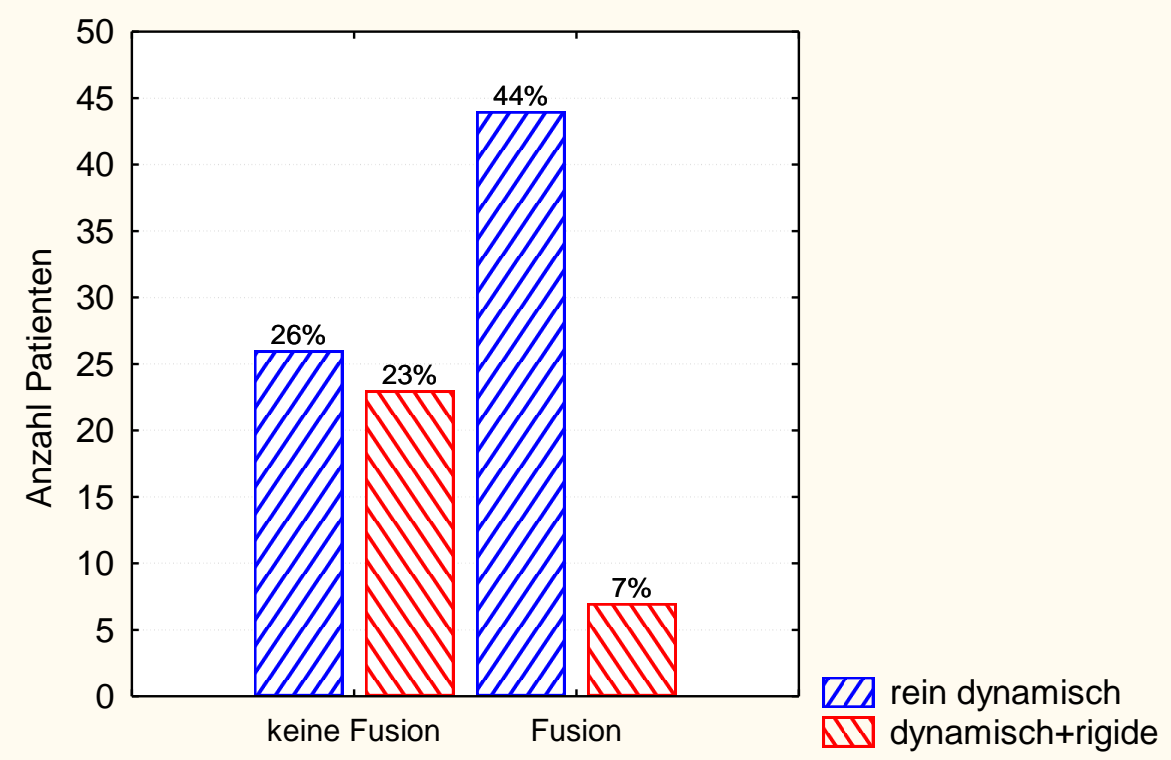

Abbildung 42: Fusionsrate bezogen auf OP-Umfang 


\subsubsection{Postoperativer Rückenschmerz bei Fusion / Erhalt Dynamik}

$22 \%$ der radiologisch fusionierten Patienten und $29 \%$ der nicht knöchern fusionierten gaben postoperative Schmerzen im Bereich von leichten bis mittleren Beschwerden an (VAS).

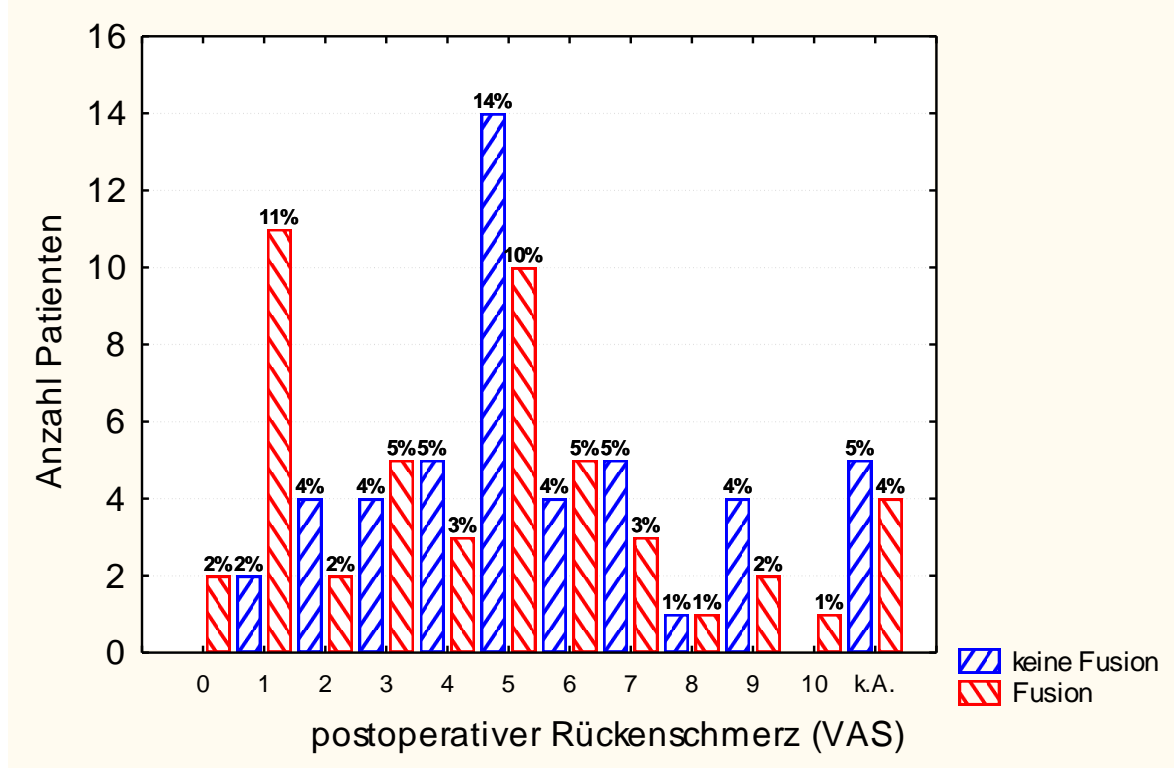

Abbildung 43: postoperativer Rückenschmerz (VAS) bezogen auf Fusion

\subsubsection{Anschlussinstabilitäten}

Bei 6 Patienten aus der Gruppe der kombiniert dynamisch und rigide fusionierten Patienten und einer Patientin mit rein dynamischer Fusion der LWS zeigte sich radiologisch eine Instabilität im kranial anschließenden Segment. 


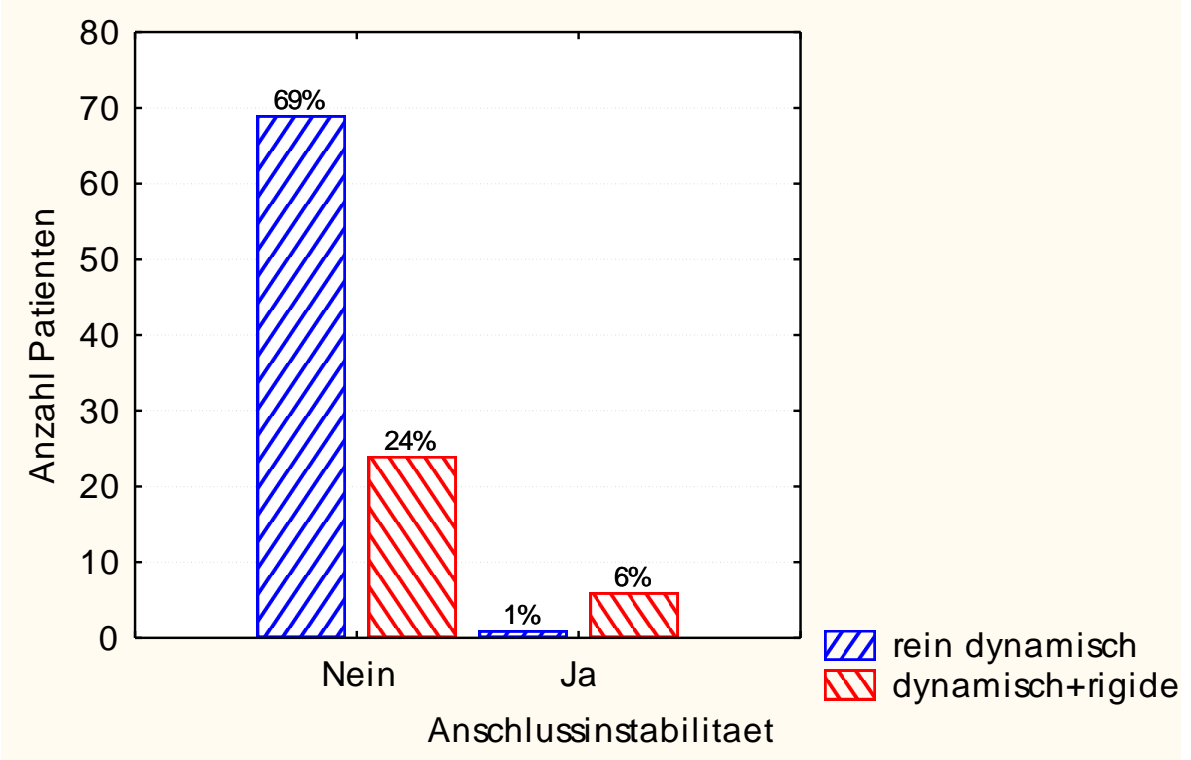

Abbildung 44: Häufigkeitsverteilung Anschlussinstabilität bezogen auf OP-Umfang

\subsubsection{Fraktur im Anschlusssegment}

Bei 4 Patienten zeigte sich eine Fraktur des kranialen Anschlusswirbels. Hierbei handelt es sich ebenfalls um Patienten, die kombiniert dynamisch und rigide instrumentiert wurden. 


\subsection{Analytische Auswertung}

\subsubsection{Einfluss des Rauchens auf die Fusionsrate}

Untersucht man die Frage, ob Rauchen einen Einfluss auf die Rate der knöchernen Fusion hat, so beobachtet man, dass es bei $64,29 \%$ der Raucher nicht zu einer Fusion kommt. Dies ist jedoch nur bei $41,79 \%$ der Nichtraucher der Fall. Bei Berechnung des $p$-Wertes erhält man $p=0,0455$ und muss somit von einem signifikanten Einfluss des Rauchens auf die Fusionsrate ausgehen.

\begin{tabular}{|c|c|c|}
\hline & Nichtraucher & Raucher \\
\hline Keine Fusion & 28 Patienten & 18 Patienten \\
\hline Fusion & 39 Patienten & 10 Patienten \\
\hline
\end{tabular}

Tabelle 6: Einfluss des Rauchens auf Fusionsrate

\begin{tabular}{|l|r|l|r|}
\hline & Spalte 1 & Spalte 2 & Zeile gesamt \\
\hline Häufigkeiten, Zeile 1 & 28 & 18 & 46 \\
\hline Prozent von Gesamt & $29,474 \%$ & $18,947 \%$ & $48,421 \%$ \\
\hline Häufigkeiten, Zeile 2 & 39 & 10 & 49 \\
\hline Prozent von Gesamt & $41,053 \%$ & $10,526 \%$ & $51,579 \%$ \\
\hline Spalten Gesamt & 67 & 28 & 95 \\
\hline Prozent von Gesamt & $70,526 \%$ & $29,474 \%$ & \\
\hline Chi-Quadrat (FG=1) & $\mathbf{4 , 0 0}$ & $\mathbf{p = 0 , 0 4 5 5}$ & \\
\hline
\end{tabular}

Tabelle 7: Chi $^{2}$-Test: Einfluss Rauchen auf Fusion 


\subsubsection{Einfluss des Alters auf die Fusionsrate}

Betrachtet man nun die Frage, ob das Alter, hier die Skalierung jünger bzw. älter als 60 Jahre, einen Einfluss auf die Rate an knöcherner Fusion besitzt, so erhält man auch hier einen p-Wert, der für Signifikanz spricht. Bei 31,25\% der jüngeren Patienten kommt es zu einer Fusion. Bei den älteren Patienten ist die Fusionsrate fast doppelt so hoch $(59,4 \%)$.

\begin{tabular}{|c|c|c|}
\hline & $\begin{array}{c}\text { Patientenalter } \\
<60 \text { Jahre }\end{array}$ & $\begin{array}{c}\text { Patientenalter } \\
>60 \text { Jahre }\end{array}$ \\
\hline Keine Fusion & 22 Patienten & 28 Patienten \\
\hline Fusion & 10 Patienten & 41 Patienten \\
\hline
\end{tabular}

Tabelle 8: Einfluss des Alters auf Fusionsrate

\begin{tabular}{|l|r|l|r|}
\hline & Spalte 1 & Spalte 2 & Zeile gesamt \\
\hline Häufigkeiten, Zeile 1 & 22 & 28 & 50 \\
\hline Prozent von Gesamt & $21,782 \%$ & $27,723 \%$ & $49,505 \%$ \\
\hline Häufigkeiten, Zeile 2 & 10 & 41 & 51 \\
\hline Prozent von Gesamt & $9,901 \%$ & $40,594 \%$ & $50,495 \%$ \\
\hline Spalten Gesamt & 32 & 69 & 101 \\
\hline Prozent von Gesamt & $31,683 \%$ & $68,317 \%$ & \\
\hline Chi-Quadrat (FG=1) & $\mathbf{6 , 9 4}$ & $\mathbf{p = 0 , 0 0 8 4}$ & \\
\hline
\end{tabular}

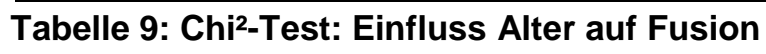




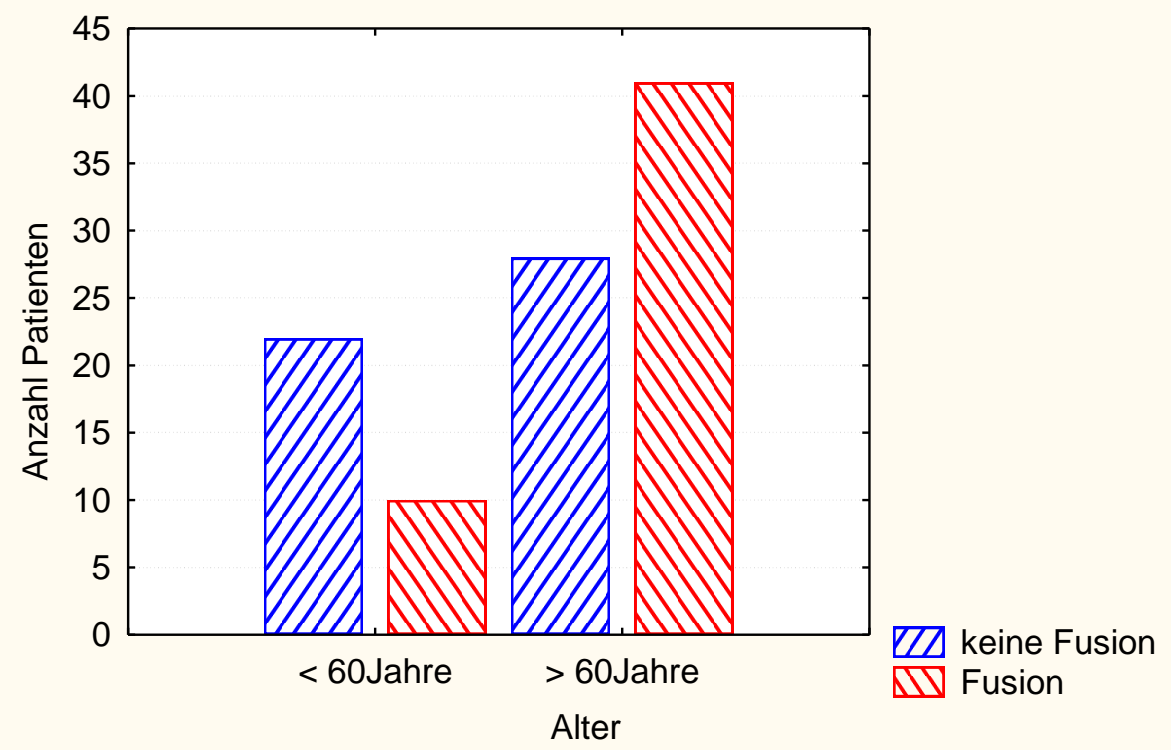

Abbildung 45: Häufigkeit Fusion bezogen auf Alter

3.3.3 Einfluss des Operationsumfanges auf das Auftreten einer Anschlussinstabilität

Eine Anschlussinstabilität im cranialen Segment entwickelten 6 Patienten der kombiniert dynamisch+rigide fusionierten Patienten, was einer Instabilitätsrate von 20\% entspricht. Im Gegensatz hierzu konnte man 1 Anschlussinstabilität bei einem rein dynamisch fusionierten Patienten beobachten. Hier beträgt die Instabilitätsrate $1,43 \%$. Mit Hilfe des $p$-Wertes $(p=0,0009)$ lässt sich ein signifikanter Unterschied der beiden untersuchten Gruppen darstellen.

\begin{tabular}{|c|c|c|}
\hline Anschlussinstabilität & Rein dynamische Fusion & $\begin{array}{c}\text { Kombiniert } \\
\text { dynamisch+rigide } \\
\text { Fusion }\end{array}$ \\
\hline Nein & 69 Patienten & 24 Patienten \\
\hline Ja & 1 Patient & 6 Patienten \\
\hline
\end{tabular}

Tabelle 10: Einfluss des OP-Umfangs auf das Auftreten einer Anschlussinstabilität 


\begin{tabular}{|l|r|r|r|}
\hline & \multicolumn{2}{|l|}{ Spalte 1 } & Zeile gesamt \\
\hline Häufigkeiten, Zeile 1 & 69 & 24 & 93 \\
\hline Prozent von Gesamt & $69,00 \%$ & $24,00 \%$ & $93,00 \%$ \\
\hline Häufigkeiten, Zeile 2 & 1 & 6 & 7 \\
\hline Prozent von Gesamt & $1,00 \%$ & $6,00 \%$ & $7,00 \%$ \\
\hline Spalten Gesamt & 70 & 30 & 100 \\
\hline Prozent von Gesamt & $70,00 \%$ & $30,00 \%$ & \\
\hline Chi-Quadrat (FG=1) & $\mathbf{1 1 , 1 3}$ & $\mathbf{p = 0 , 0 0 0 9}$ & \\
\hline
\end{tabular}

Tabelle 11: Chi'2-Test: Einfluss OP-Umfang auf Anschlussinstabilität

3.3.4 Postoperativer Schmerzmittelbedarf bei Anschlussinstabilität im Vergleich zum präoperativen Schmerzmittelbedarf

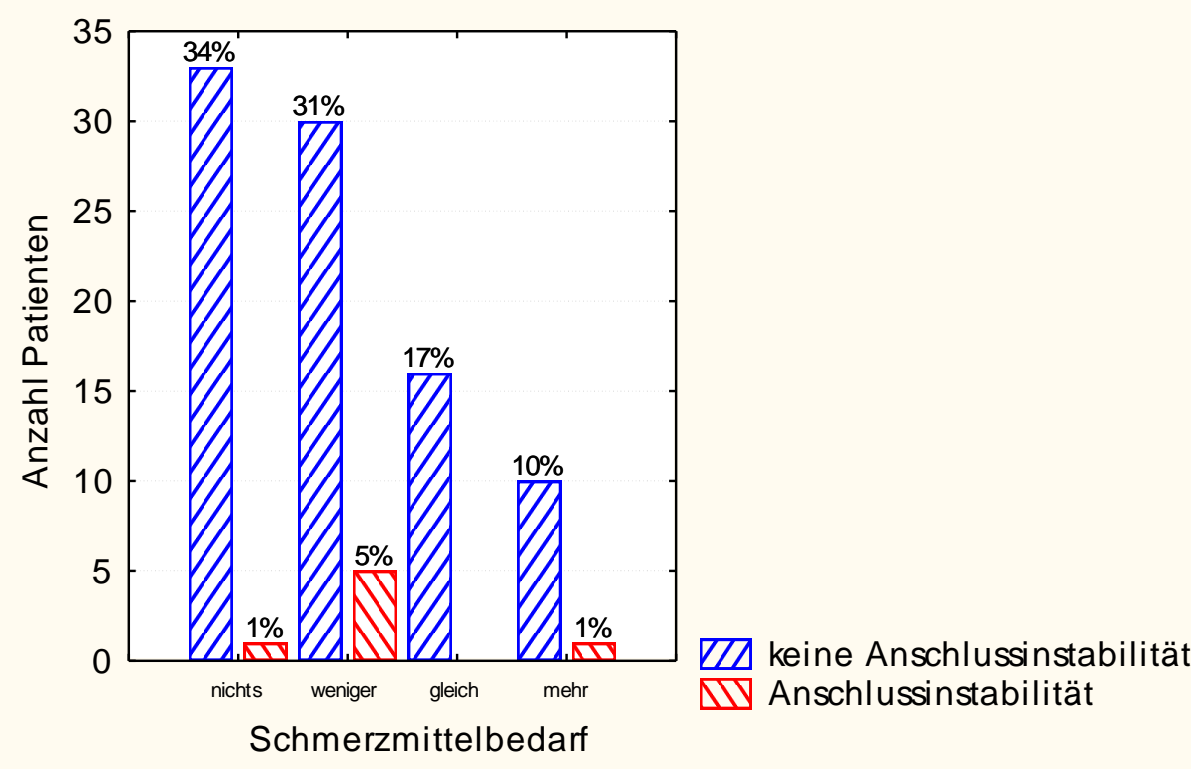

Abbildung 46: postoperativer Schmerzmittelbedarf im Vergleich zu präoperativ unter Berücksichtigung Anschlussinstabilität 
Ergebnisse

\subsubsection{Einfluss des Rauchens auf das Auftreten einer Schraubenlockerung}

Eine Schraubenlockerung konnte bei $10,7 \%$ der rauchenden Patienten und bei 23,5\% der Nichtraucher gesehen werden. Mit Hilfe der p-Wert-Berechnung lässt dies keinen Schluss auf einen signifikanten Einfluss zu.

\begin{tabular}{|c|c|c|}
\hline & Nichtraucher & Raucher \\
\hline Keine & 52 Patienten & 25 Patienten \\
Schraubenlockerung & & \\
\hline Schraubenlockerung & 16 Patienten & 3 Patienten \\
\hline
\end{tabular}

Tabelle 12: Einfluss Rauchen auf Schraubenlockerung

\begin{tabular}{|l|r|l|r|}
\hline & Spalte 1 & Spalte 2 & Zeile gesamt \\
\hline Häufigkeiten, Zeile 1 & 52 & 25 & 77 \\
\hline Prozent von Gesamt & $54,167 \%$ & $26,042 \%$ & $80,208 \%$ \\
\hline Häufigkeiten, Zeile 2 & 16 & 3 & 19 \\
\hline Prozent von Gesamt & $16,667 \%$ & $3,125 \%$ & $19,792 \%$ \\
\hline Spalten Gesamt & 68 & 28 & 96 \\
\hline Prozent von Gesamt & $70,833 \%$ & $29,167 \%$ & \\
\hline Chi-Quadrat (FG=1) & $\mathbf{2 , 0 5}$ & $\mathbf{p = 0 , 1 5 2 0}$ & \\
\hline
\end{tabular}

Tabelle 13: Chi²-Test: Einfluss Rauchen auf Schraubenlockerung 


\subsubsection{Einfluss einer Schraubenlockerung auf die Fusionsrate}

Dabei erhielt man für die Frage nach der Signifikanz des Einflusses einer Schraubenlockerung auf die Fusion und die Frage des Einflusses der Dekompression auf den postoperativen Beinschmerz einen $p$-Wert $<0,05$. Dies spricht jeweils für einen signifikanten Einfluss.

\begin{tabular}{|c|c|c|c|}
\hline $\begin{array}{c}\text { Untersuchte } \\
\text { Variable }\end{array}$ & $\begin{array}{c}\text { angenommener } \\
\text { Einflussfaktor }\end{array}$ & p-Wert & Signifikanz \\
\hline Schmerzmittelbedarf & Fusion & 0,2797 & nicht signifikant \\
\hline $\begin{array}{c}\text { Postoperativer } \\
\text { Rückenschmerz }\end{array}$ & Operationszugang & 0,5427 & nicht signifikant \\
\hline $\begin{array}{c}\text { Postoperativer } \\
\text { Rückenschmerz }\end{array}$ & Schraubenlockerung & 0,1914 & nicht signifikant \\
\hline $\begin{array}{c}\text { Postoperativer } \\
\text { Rückenschmerz }\end{array}$ & Voroperation & 0,6351 & nicht signifikant \\
\hline $\begin{array}{c}\text { Bewertung } \\
\text { Oswestry-Score }\end{array}$ & Voroperation & 0,2283 & nicht signifikant \\
\hline $\begin{array}{c}\text { Zufriedenheit mit } \\
\text { jetziger Situation }\end{array}$ & Voroperation & 0,6584 & nicht signifikant \\
\hline $\begin{array}{c}\text { Postoperativer } \\
\text { Rückenschmerz }\end{array}$ & Fusion & 0,0660 & nicht signifikant \\
\hline Fusion & Schraubenlockerung & $\mathbf{0 , 0 1 6 9}$ & signifikant \\
\hline $\begin{array}{c}\text { Postoperativer } \\
\text { Beinschmerz }\end{array}$ & Dekompression & $\mathbf{0 , 0 2 6 9}$ & signifikant \\
\hline
\end{tabular}

Tabelle 14: P-Werte, Mann-Whitney-U-Test 


\begin{tabular}{|c|c|c|c|c|c|c|c|c|c|c|}
\hline & Nach Varia & le Schraube & lockeru & & & & & & & \\
\hline Variable & $\begin{array}{l}\text { Rgsumme } \\
\text { Gruppe } 1\end{array}$ & $\begin{array}{l}\text { Rgsumme } \\
\text { Gruppe } 2\end{array}$ & $U$ & Z & $p$-Wert & $\begin{array}{c}\text { Z } \\
\text { korr. }\end{array}$ & p-Wert & $\begin{array}{c}\text { Gült. N } \\
\text { Gruppe } \\
1\end{array}$ & $\begin{array}{c}\text { Gült. N } \\
\text { Gruppe } \\
2\end{array}$ & $\begin{array}{c}2^{*} \text { eins } \\
\text { Exakt } \\
p\end{array}$ \\
\hline Fusion & 3960,00 & 696,00 & 506,00 & 2,06902 & 0,03854 & 2,389490 & 0,01687 & 77 & 19 & 0,03783 \\
\hline
\end{tabular}

Tabelle 15: Mann-Whitney-U-Test: Einfluss Schraubenlockerung auf Fusion

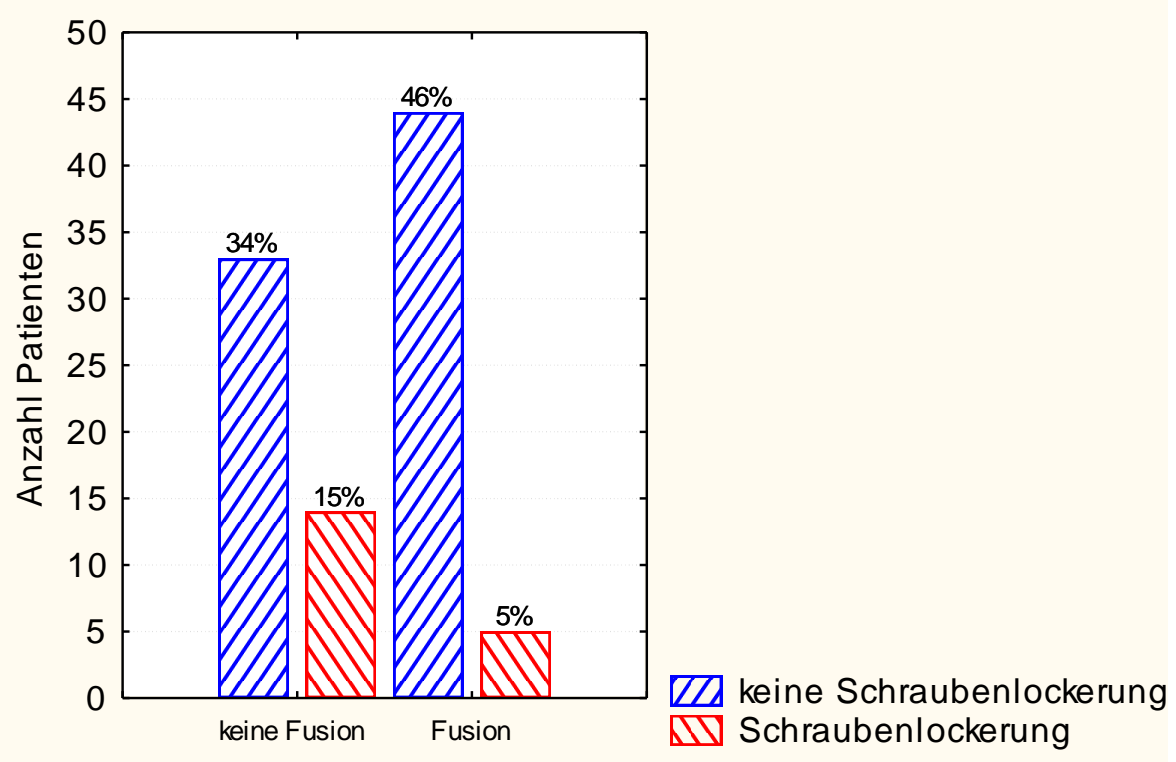

Abbildung 47: Häufigkeitsverteilung Fusion bezogen auf Schraubenlockerung

Patienten, die eine radiologisch nachweisbare Schraubenlockerung aufwiesen, zeigten eher keine knöcherne Fusion der Facettengelenke. 


\begin{tabular}{|c|c|c|c|c|c|c|c|c|c|c|}
\hline \multirow[b]{2}{*}{ Variable } & \multicolumn{10}{|c|}{ Nach Variable Dekompression } \\
\hline & $\begin{array}{c}\text { Rgsumme } \\
\text { Gruppe } 1\end{array}$ & $\begin{array}{c}\text { Rgsumme } \\
\text { Gruppe } 2\end{array}$ & $\mathrm{U}$ & Z & p-Wert & $\begin{array}{c}\mathrm{Z} \\
\text { korr. }\end{array}$ & p-Wert & $\begin{array}{c}\text { Gült. N } \\
\text { Gruppe } \\
1\end{array}$ & $\begin{array}{c}\text { Gült. N } \\
\text { Gruppe } \\
2\end{array}$ & $\begin{array}{l}2^{*} \text { eins } \\
\text { Exakt } p\end{array}$ \\
\hline $\begin{array}{l}\text { postOP- } \\
\text { Beinschmerz }\end{array}$ & 4144,50 & 805,50 & 316,500 & $-2,198$ & 0,02796 & $-2,2146$ & 0,02678 & 87 & 12 & 0,02628 \\
\hline
\end{tabular}

Tabelle 16: Mann-Whitney-U-Test: Einfluss Dekompression auf postoperative Ischialgie

Ein Einfluss der Dekompression auf die Stärke der postoperative Ischialgie konnte mit Hilfe des Mann-Whitney-U-Tests nachgewiesen werden.

3.3.7 Einfluss von Alter und / oder BMI auf Schraubenbruch, -lockerung, Anschlussinstabilität, Fusion, Schmerzmittelbedarf

\begin{tabular}{|c|c|c|c|}
\hline $\begin{array}{c}\text { Untersuchte } \\
\text { Variable }\end{array}$ & $\begin{array}{c}\text { angenommener } \\
\text { Einflussfaktor }\end{array}$ & p-Wert & Signifikanz \\
\hline Fusion & BMI & 0,5259 & nicht signifikant \\
\hline Fusion & Alter & $\mathbf{0 , 0 0 1 1}$ & signifikant \\
\hline Schraubenbruch & BMI & 0,8364 & nicht signifikant \\
\hline Schraubenlockerung & BMl & 0,7933 & nicht signifikant \\
\hline Anschlussinstabilität & BMI & 0,5558 & nicht signifikant \\
\hline Schraubenlockerung & Alter & 0,1182 & nicht signifikant \\
\hline Schmerzmittelbedarf & Alter & 0,1807 & nicht signifikant \\
\hline
\end{tabular}

Tabelle 17: P-Werte Logistische Regression

Wie auch schon im Chi²-Test, kann mit Hilfe der logistischen Regression ein signifikanter Unterschied der Patienten in den beiden Altersgruppen, jünger bzw. älter als 60 Jahre, bezüglich des Auftretens einer knöchernen Fusion gezeigt werden. 
Ergebnisse

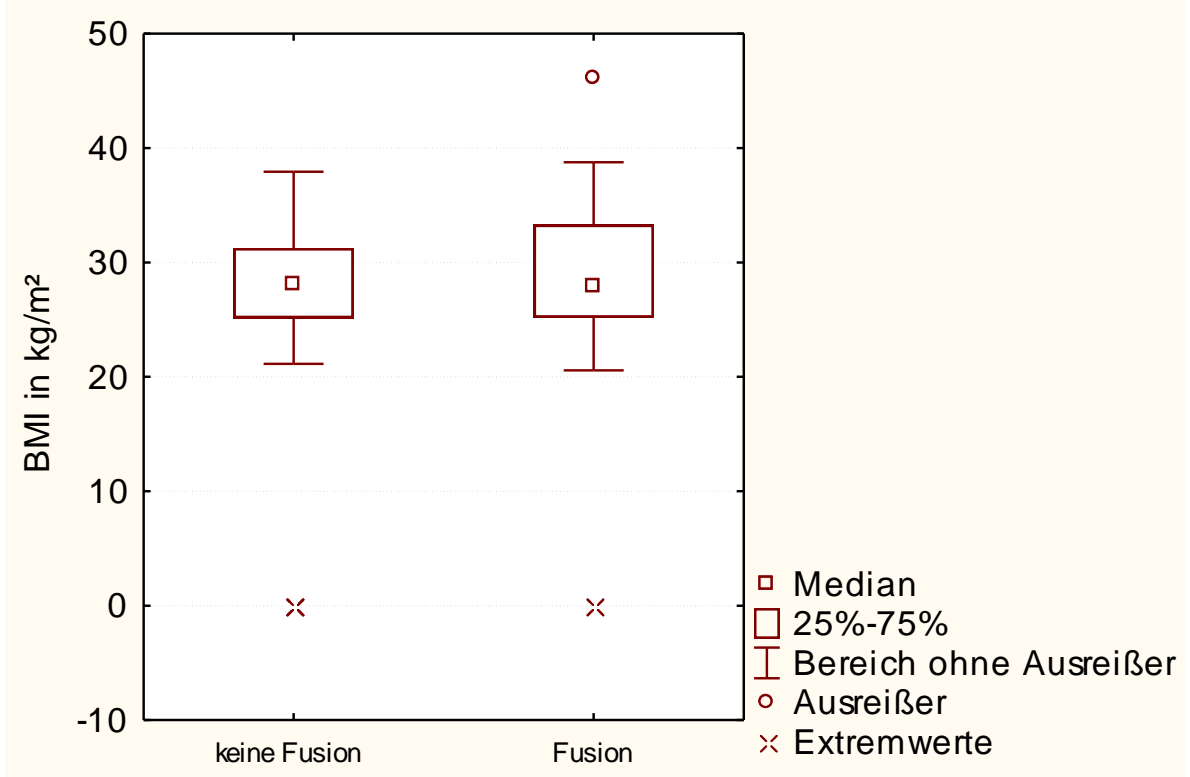

Abbildung 48: Fusion bezogen auf BMI in $\mathrm{kg} / \mathrm{m}^{2}$

Ein Einfluss der Höhe des Body-Mass-Indexes auf die Rate der knöchernen Fusion konnte nicht ermittelt werden. 


\subsection{Komplikationen}

Im intraoperativen Verlauf kam es bei 7 Patienten zu einer Duraverletzung mit Liquorleck, welches sofort mit Tachosilß abgedeckt wurde. 2 Patienten erhielten wegen persisterendem Liquoraustritt eine Revisionsoperation. Intraoperative Gefäßund Nervenverletzungen wurden nicht gesehen. Desweiteren konnten keine funktionell relevanten Implantatfehllagen beobachtet werden. Ein Patient wurde während der direkten postoperativen Phase im Aufwachraum aufgrund einer Asystolie reanimiert. Ein weiterer Patient verstarb am 5. postoperativen Tag im Rahmen einer stattgehabten Lungenarterienembolie unter Reanimationsbedingungen. Eine Patientin wies eine transfusionsbedürftige Blutungsanämie auf. Bei einem Patienten entwickelte sich eine Pneumonie und drei Patienten litten unter Wundheilungsstörungen, wovon ein Patient revidiert wurde.

\subsection{Dropouts}

Von den zu Beginn der Studie telefonisch eingeladenen 120 Patienten nahmen 99 Patienten den Termin zur Nachuntersuchung wahr. 10 Patienten konnten telefonisch nicht erreicht werden, da sie unbekannt verzogen sind, 6 Patienten verweigerten die Teilnahme an der Nachuntersuchung. Während der ersten 12 postoperativen Monate verstarben 2 Patienten. In 3 Fällen musste eine Revisionsoperation durchgeführt werden, in der das dynamische System entfernt und durch eine rigide Versorgung ersetzt wurde. 


\section{Diskussion}

Im Rahmen der natürlichen Degeneration des spinalen Bewegungssegments entwickeln sich neben Bandscheibenveränderungen, deren klinischer Einfluss leider bislang nur schlecht verstanden wird, auch Veränderungen im Bereich der Facettengelenke und der angrenzenden Bandstrukturen (Quint et al. 1998).

Folgen der entstehenden Dysfunktion im Bewegungssegment sind die Entstehung spinaler Engen (Spinalkanalstenose) und häufig das Auftreten einer degenerativen Instabilität (Spondylolisthese).

Die klassische Behandlung dieser degenerativen Veränderungen umfasst die Dekompression der Stenose und die in der Regel instrumentierte Fusion und somit Stabilisierung des Bewegungssegments als Goldstandard (Gibson und Waddell 2005, Schulte et al. 2006, Postacchini und Cinotti 1992, Weinstein et al. 2008).

Für Behandlung von Instabilitäten der Wirbelsäule sind in der Historie der Wirbelsäulenchirurgie verschiedenste Operationstechniken entwickelt worden, die zu ebendieser Stabilisierung der Bewegungssegmente beitragen. Nach Strategien, mit autologer Knochentransplantation alleine eine Verknöcherung und somit Fusion zu erzielen, erschienen in der Folge Instrumentationen, die über eine zusätzliche Einschränkung des Bewegungsumfanges zu einer dauerhaften knöcherner Stabilisierung führen sollen (Niosi et al. 2006).

Der Einsatz von Zwischenwirbelimplantaten in Gestalt wiederum von autologer Knochentransplantation oder in der Folge die Entwicklung von Platzhaltern (Cages) aus Fremdmaterialien wie Titan oder PEEK hat zu weiter verbesserten Erfolgen in Form von höheren Fusionsraten geführt. 


\subsection{Kritik an rigiden Fusionssystemen}

Trotz mittlerweile guter Fusionsraten von bis zu 95\% hat sich hinsichtlich der klinischen Ergebnisse solcher Behandlungsstrategien in den letzten Jahren keine entscheidende Tendenz zur Besserung gezeigt (Etebar und Cahill 1999), so dass in größeren Übersichtsarbeiten die Rate zufriedenstellender Outcomes von $16 \%$ bis 95\% reichen (Turner et al. 1992, Boos und Webb 1997, Resnick et al. 2005, Sengupta und Mulholland 2005).

\subsubsection{Anschlussinstabilitäten und -frakturen}

Die Ruhigstellung des schmerzverursachenden Segments verursacht jedoch eine Mehrbelastung im angrenzenden Segment, was wiederum neue Instabilität und Schmerzen verursachen kann. So berichten einige Studien über Anschlussinstabilitäten und gar Frakturen in diesen der Fusion benachbarten Bewegungssegmenten (Fritzell et al. 2004, Gibson et al. 1999, Glaser et al. 2003, Möller und Hedlund 2000, Mummaneni et al. 2004).

\subsubsection{Operationsmorbidität}

Ein weiterer Kritikpunkt besteht in der im Vergleich zu rein transpedikulären Fusionsverfahren erhöhten Operationsmorbidität. Eine längere Operationszeit für die Ergänzung der interkorporellen Spondylodese (von dorsal oder ventral) wie gegebenenfalls Beschwerden im Bereich einer Knochenspanentnahme erhöhen das perioperative Risiko wie das postoperative Gesamtbeschwerdeniveau. 


\subsection{Die Entwicklung dynamischer transpedikulärer Systeme. Vorteile.}

Um die Belastung der angrenzenden Segmente möglichst gering zu halten, das Bewegungsausmaß im behandelten Segment jedoch ausreichend stabilisieren zu können, wurden die beweglichen (dynamischen) transpedikulären Systeme entwickelt (Gédet et al. 2009). Die Beweglichkeit des Systems soll zum einen die Beweglichkeit im versorgten Segment auf ein schmerzlinderndes Ausmaß einschränken. Zum anderen wird durch den Verzicht auf eine interkorporelle Spondylodese (wie Cage oder autologen Knochen) auch die perioperative Belastung des Patienten reduziert (geringere OP-Dauer, keine zusätzliche Knochenspanentnahme).

\subsection{Anforderung an dynamische transpedikuläre Systeme, Ergebnisse in der Verwendung des DSS}

In biomechanischen Studien konnte in den letzten Jahren für einige dynamische transpedikuläre Systeme gezeigt werden, dass sie in der Lage sind, den Bewegungsumfang im behandelten Segment messbar einzuschränken (Schulte et al. 2008, Schmoelz et al. 2006, Grevitt et al. 1995, Schmoelz et al. 2009).

Korovessis et al. konnten 2004 in ihren Untersuchungen keine klinischen Unterschiede im Outcome im Vergleich zwischen rigiden und dynamischen transpedikulären Versorgungsstrategien erkennen.

Nach Beobachtungen von Fischgrund et al. 1997 sowie Herkowitz und Kurz 1991 werden auch in der Verwendung von rigiden Fusionssystemen bei Pseudarthrosen und somit ausbleibender knöcherner Fusion zum Teil gute bis sehr gute Ergebnisse erzielt. 
Die Anforderung an ein dynamisches transpedikuläres Fusionssystem umfassen somit eine ausreichende Einschränkung des Bewegungsumfanges im dysfunktionalen Segment sowie eine im Vergleich zu rigiden Fusionssystemen vergleichbare Rate an Implantatversagen. Klinische Ergebnisse müssen mindestens den gleichen Erfolg wie rigide Stabilisierungssysteme aufweisen.

\subsubsection{Schraubenlockerungsraten, Implantatbrüche}

Rigide transpedikuläre Fusionssysteme zeigen Schraubenlockerungs- und bruchraten von etwa 17\% (Schnake et al. 2006). In der vorliegenden Studie wurde eine Rate von Implantatversagen hinsichtlich Schraubenlockerung von $5,4 \%$ bei dynamischen (von 202 Schrauben) 8,8\% bei rigiden Schrauben (260) beobachtet. Die Bruchrate lag bei 0,8\% der verwendeten Schrauben.

Insgesamt ergibt sich hieraus eine geringere Rate an Implantatversagen in der dynamischen Schraubengruppe verglichen mit Exemplaren der rigiden Versorgung im Rahmen der behandelten Patientengruppe.

Im Vergleich zu anderen Studien fällt auf, dass die Beschreibung des Grundes für das Implantatversagen sehr rudimentär ausfällt. So berechnen beispielsweise Lonstein et al. 1999 eine „allgemeine Komplikationsrate“ von 2,4\% pro Schraube bei 4790 implantierten Schrauben (Young et al. 2007). Andere Autoren machen nur sehr allgemeine Angaben wie Bruchraten ohne die Beschreibung von Lockerungszahlen.

Ein weiterer Kritikpunkt stellt die häufig sehr geringe Patientenzahl von Studien dar. Cakir beschreibt zum Beispiel 2003 eine Serie von 10 Patienten mit dynamischer transpedikulärer Fusion ohne Implantatversagen, Schaeren et al. verfolgten in Ihrer Darstellung 2008 gerade 26 Patienten hinsichtlich der genannten Komplikationen alle Teilnehmer der Untersuchung wurden alleine nativradiologisch nachuntersucht, was auch aufgrund der deutlich geringeren Sensitivität dieses diagnostischen Verfahrens natürlich kritisch gesehen werden muss. 


\subsubsection{Biomechanische Veränderungen nach Fusion}

\subsubsection{Anschlussinstabilitäten im Allgemeinen}

Die genaue Ursache für Anschlussinstabilitäten ist bislang noch nicht recht geklärt. Ein Faktor, der im Anschluss an rigide Fusionen hierzu wohl beiträgt, ist die Verschiebung des Rotationszentrums nach dorsal, was die Belastung der Facettengelenke auch in den angrenzenden Bewegungssegmenten erhöht (Lee CK 1988, Highsmith et al. 2007) und somit zur beschleunigten Degeneration beiträgt.

Natürlich existieren auch andere operationsbedingte Einflüsse, die zu forcierter Degeneration von Anschlusssegmenten beitragen wie die Verletzung benachbarter (cranial gelegener) Gelenkkapseln beim Einbringen der Schrauben, muskuläre Denervierung durch den mehr oder minder traumatisierenden operativen Zugang, Überdistraktion und dadurch iatrogene Kyphosierung und Ähnliches.

Auch Cadaverstudien eignen sich nicht in jedem Fall zur Ergebnisübertragung auf den funktionierenden Komplex Wirbelsäule mit angrenzenden Strukturen. Untersuchungen über die Mehrbelastung angrenzender Bewegungssegmente zu dynamisch fusionierten sind natürlichen Limitierungen unterworfen. So ist es nicht möglich, in vitro oben genannte Effekte wie posturale Störungen des Patienten, Fehlpositionierung (Abweichung von der idealisierten Implantationsrichtung und Stellung der Implantate, Verletzung der angrenzenden Facettengelenke durch die Pedikelschrauben etc.), Fehler in der Auswahl der Implantatgröße, physiologische Belastungsmuster, Einfluss der Muskulatur (auch unter dynamischen Bedingungen wie natürlicher Hypo- wie Hypertrophie, Scherkräfte etc.), Einfluss wechselnder Temperatur auf die verwendeten Materialien (Ermüdungsphänomene), Einfluss von Narbengewebe auf die Biomechanik des behandelten Segments sowie der 
angrenzenden einzuschätzen (Lafage et al. 2007). Ebenso bleibt das Ausmaß der zugrundeliegenden degenerativen Gesamtentwicklung der Wirbelsäule unbeurteilt und dessen Einfluss daher unverstanden bzw. zumindest nicht objektiv einschätzbar. Pellisé et al. zeigten 2007, dass Bandscheibendegenerationen in mehreren kranialen Anschlusssegmenten lumbaler Fusionen eintreten und voranschreiten. Ein unmittelbarer Zusammenhang zur stattgehabten Fusion ist daher nicht zweifelsfrei anzunehmen (Cheng et al. 2007).

Zudem erlauben einige in vitro (Kadaverstudie) Studiendesigns nicht einmal mehr eine Beurteilung physiologischer Bedingungen im Kadaver. So erfordert eine Messung von Lastgrößen zwischen Gelenkfacetten eine Eröffnung der Gelenkkapsel (Niosi et al. 2008). Durch diese Einflussnahme entstehen Veränderungen, die signifikant Einfluss haben können auf Faktoren wie den Bewegungsumfang und die damit verbundenen wirkenden Kräfte.

Hinsichtlich der Häufigkeit im Auftreten von Anschlussinstabilitäten existieren sehr heterogene Ergebnisse nach aktueller Datenlage. Nachdem eine der initialen Anforderungen an ein dynamisches Versorgungskonzept die Reduktion von Anschlussinstabilitäten war, konnten diese Erwartungen nicht sicher bestätigt werden. Die Rate der Instabilitäten in den Nachbarsegmenten zeigte ähnliche Ergebnisse wie im Falle rigider Fusionen (Rahm und Hall 1996, Cakir et al. 2009). Hier wurden Anschlussinstabilitäten in bis zu 35\% der Fälle beobachtet. Schaeren et al. berichteten 2008 von 47\% Veränderungen dieser Art im cranialen Anschlusssegment.

In der vorliegenden Untersuchung konnte in der Verwendung des dynamischen transpedikulären Fusionssystems DSS eine Rate von 7\% Anschlussinstabilitäten beobachtet werden. Hierzu muss auf die unterschiedlichen technischen Voraussetzungen eingegangen werden: Während das am häufigsten in der Literatur untersuchte transpedikuläre System aus Titanschrauben besteht, die durch Polyesterkordeln in einem hohlen Platzhalter miteinander verbunden sind (Dynesys, Zimmer Spine), beruht das verwendete System auf einer Titanverbindung zwischen 
den Schrauben. Möglicherweise besteht hier ein entscheidender Unterschied im Vergleich der beiden Systeme.

\subsubsection{Knöcherne Fusion im dynamisch versorgten Segment}

In der Auswertung der radiologischen Ergebnisse zeigte sich in 51\% der Fälle einer knöcherne Fusion im ursprünglich ja dynamisch versorgten Segment.

Hierbei konnte in der Gruppe der über 60-Jährigen eine fast doppelt so hohe Rate an Fusionen beobachtet werden.

Diese Beobachtung ist in der aktuellen Literatur so nicht regelhaft $z u$ finden und scheint einen neuen Aspekt aufzuwerfen. Hier ist das grundlegende Prinzip, die Beweglichkeit im Segment zu erhalten, zwangsweise verlassen und das Ergebnis einer rigiden Fusion erzielt. $\mathrm{Zu}$ den günstigeren Bedingungen des dynamischen Konzepts mit kürzerer Operationsdauer und geringerer Operationsmorbidität wird hierbei das Ergebnis eines aufwendigeren Verfahrens erreicht.

In diesem Rahmen kann ein weiterer Zusammenhang angenommen werden. Möglicherweise führt die langsam zunehmende Versteifung durch die ursprünglich dynamische Verbindung ähnlich wie bei der nichtinstrumentierten Wiltsefusion aufgrund der langsam zunehmenden Einschränkung im Bewegungsumfang zu einer Art Anpassung der angrenzenden Segmente. Der als Slow Fusion bezeichnete Prozess könnte als Äquivalent einer physiologischen, möglicherweise spondylophytär bedingten Versteifung zu einer geringeren Rate an Anschlussinstabilitäten führen.

Bei Rauchern bestätigt sich nach den vorliegenden Ergebnissen die bekannte Beobachtung einer schlechteren Fusionsrate als Beispiel für Knochenheilung (CesarNeto et al. 2003). Bei 28 Rauchern wurden 10 knöcherne Fusionen gesehen $(35,7 \%)$. Nichtraucher fusionieren eher $(58,2 \%)$. 


\subsection{Klinische Ergebnisse: Outcome-Beurteilung}

Daten bezüglich des klinischen Outcomes von Patienten werden meist durch die Erhebung des postoperativen Schmerzniveaus anhand der Visuellen Analogskala (VAS) sowie eines Fragebogens zur Einschätzung der empfundenen Behinderung, in vielen Fällen des Oswestry Disability Index, erhoben (Ostelo et al. 2008). Die Höhe der Schmerzpunktzahl auf der Visuellen Analogskala sinkt nach Dekompression und Stabilisierung im Bereich der LWS postoperativ signifikant (Schnake et al. 2006, Würgler-Hauri et al. 2008, Vaga et al. 2009). Die Aussage wird ebenso für die Auswertung des Oswestry-Disability-Index getroffen (Yee et al. 2008, Würgler-Hauri et al. 2008, Welch et al. 2007).

Dies muss jedoch kritisch gesehen, werden, da Patienten selbständig einen subjektiven Fragebogen ausfüllen (Pellisé et al. 2005). So hängt die Einschätzung des postoperativen Zustandes von vielen Einflussfaktoren, wie z.B. Alter und Geschlecht ab. Ebenso beeinflusst die persönliche Erwartung an die Operation das Outcome (Yee et al. 2008). Einige Autoren sagen, dass je schlechter der präoperative Gesamtzustand eingeschätzt wird, desto weniger Verbesserung wird postoperativ angegeben (Jönsson et al. 1997, Katz et al. 1999). Auch der psychische Zustand eines Patienten zum Zeitpunkt der Datenerhebung oder eine vorbekannte Depression wirken sich auf die Selbsteinschätzung des Patienten aus (Hazard et al. 1991, Carragee et al. 2005). Auch in der vorliegenden Studie muss man den Einfluss der Multimorbidität, bei einem Durchschnittsalter des untersuchten Patientenkollektivs von 65,5Jahren, auf die Bewertung der individuellen postoperativen Situation berücksichtigen.

So berichteten fast ein Drittel der Patienten, dass es ihnen schwer falle, ihre Beschwerden gezielt der Wirbelsäule zuzuordnen, da sie unter weiteren degenerativen Erkrankungen des Bewegungsapparates litten und dies zu einer Vermischung der Beschwerdesymptomatik führe. Auch sollte man bei der Bewertung der angegebenen Schmerzscore-Punkte bedenken, dass einige Patienten aufgrund anderer Erkrankungen unter einer Dauermedikation mit Analgetika stehen, die sich ja dann auch auf die von der Wirbelsäule ausgehende Schmerzsituation auswirken. 
In der vorliegenden Untersuchung fiel auf, dass jüngere Patienten (< 60 Jahre) ihren postoperativen Gesamtzustand häufiger schlechter einschätzen als die Konstituenten der Gruppe der Älteren (>60 Jahre). Möglicherweise spielen hier psychosoziale Faktoren, wie z.B. starke Belastung im beruflichen wie privaten Bereich, eine Rolle. Auch die Krankheitseinsicht und damit deren Bewältigung scheint hier erschwert zu sein.

Patienten, die zusätzlich zur Fusionsoperation eine Dekompression einer Spinalkanalstenose erhielten, schätzten ihren postoperativen Zustand durch die Abnahme der Ischialgie als deutlicher gebessert ein, als Patienten, die nur eine Fusion der Segmente erhielten. Die Dekompression alleine führt zwar nicht zur Veränderung der degenerativen Prozesse, jedoch zur Verbesserung der Lebensqualität (Würgler-Hauri et al. 2008, Schnake et al. 2006, Grob et al. 1995, DiPierro et al. 1996, Deyo et al. 1993, Martin et al. 2007).

Auch wird in der Literatur davon ausgegangen, dass ein Implantatversagen keine Auswirkung auf die Zufriedenheit des Patienten habe (Schnake et al. 2006, Schaeren et al. 2008), was auch bei der Erhebung der klinischen Daten im Rahmen dieser Nachuntersuchung gesehen werden konnte. So musste sich keiner der 20 Patienten mit einer Schraubenlockerung aufgrund von persistierenden Beschwerden einer Schraubenrevisionsoperation unterziehen, was hier natürlich auch grundsätzlich die Indikationsstellung für eine Revision in Frage stellt. Somit zeigt sich, dass die alleinige radiologische Kontrolle die klinische Untersuchung mit Befragung des Patienten nicht ersetzen kann. Denn, wie auch von Sirvanci et al 2008 beschrieben korrelieren die radiologisch erhobenen Befunde nicht zwangsläufig mit der klinischen Symptomatik des Patienten. Hier ist die Anwendung von kurzen, vom Patienten selbst auszufüllenden Fragebögen, ein adäquates Mittel zur Beurteilung von Rückenschmerzen und deren Auswirkung auf den Patienten (Herno et al. 1994 Davidson und Keating 2002). 
Aufgefallen ist jedoch, dass sich die Angaben zur Gesamtzufriedenheit zum Teil im Anamnesegespräch von den Angaben durch die Fragebögen unterscheidet. So geben nur $48 \%$ der Befragten an, dass sie mit der postoperativen Gesamtsituation zufrieden seien. $82 \%$ der Patienten würden sich jedoch auch nach Ablauf des ersten postoperativen Jahres und Berücksichtigung des Outcomes wieder für die Operation entscheiden. In aktuellen Studien wird die allgemeine Patientenzufriedenheit mit 71 $86 \%$ angegeben (Toyone et al. 2005). Schnake et al. (2006) zeigen eine Rate von $87,5 \%$ der Patienten an, die sich erneut für die Operation entscheiden würden. Insgesamt gesehen ist die präoperative Aufklärung des Patienten bezüglich der zu erwarteten postoperativen Besserung der wichtigste Faktor für die Einschätzung des Outcomes des Patienten (Yee et al. 2008).

Auch wurde die Vermutung aufgestellt, dass es bei jüngeren Patienten durch deren höhere Aktivität zu einer größeren Anzahl von Implantatversagen kommt (Schnake et al. 2006), obwohl dies nicht durch Daten belegt werden konnte.

Im Gegensatz hierzu wurde von Lawthorne III et al. (2009) angegeben, dass höheres Alter und Osteoporose als limitierend auf die Verwendung von dynamischen Systemen zu sehen sind. Die Ergebnisse dieser Arbeit stützen diese Einschätzung nicht. Im Gegenteil scheint nach den vorliegenden Untersuchungsbeobachtungen sogar gerade die Gruppe der über 60-Jährigen im Besonderen für eine dynamische Versorgungsstrategie in Form von pedikelschraubenbasierten Systemen geeignet. Eine offenbar hohe Neigung zur Verknöcherung im versorgten Segment führt im Sinne einer Slow Fusion zu einer vollständigen Stabilisierung mit im Vergleich zur primären $360^{\circ}$-Fusion reduzierter Operationszeit, geringerer Operationsmorbidität und geringeren Kosten auf Implantatseite wie für Behandlungskosten im Allgemeinen (OP-Ressourcen, schnellere Mobilisierbarkeit etc.). 


\section{Zusammenfassung}

In der vorliegenden prospektiven Untersuchung zur Verwendbarkeit und zu klinischen wie radiologischen Ergebnissen dynamischer transpedikulärer Fusionssysteme am Beispiel des Bricon DSS wurden 120 Patienten über einen Zeitraum von 12 Monaten beobachtet und eine abschließende Datenerhebung durchgeführt.

Hierzu erfolgte zum Nachuntersuchungszeitpunkt eine native Funktionsröntgenuntersuchung sowie eine Computertomografie der Lendenwirbelsäule. Die klinische Beurteilung wurde nach dem Oswestry-DisabilityIndex, dem modifizierten Pationnaire-Bogen und einem eigenen Fragebogen erstellt. Hierbei zeigten sich systembedingte Komplikationen wie Implantatlockerungen in $5,4 \%$ bei dynamischen Schrauben. Die Rate von Anschlussinstabilitäten lag bei $7 \%$. Im Literaturvergleich liegen diese Beobachtungen unter dem Durchschnitt.

Die Patientenzufriedenheit nach operativer Versorgung liegt im vergleichbaren Bereich zur aktuellen Gesamtdatenlage. Während sich die allgemeine Zufriedenheit der Patienten eher etwas unter dem Literaturdurchschnitt bewegt, ist jedoch die Motivation, sich erneut einer solchen Operation zu unterziehen, quasi gleich der publizierten Quote.

Auffallend in der aktuellen Untersuchung ist die Diagnose einer hohen knöchernen Fusionsrate im Patientengut. 51\% der Patienten zeigten in den radiologischen Kontrolluntersuchungen eine Fusion im eigentlich ja dynamisch versorgten Segment. Damit ist der ursprüngliche Gedanke einer bewegungserhaltenden Stabilisierung zwar verlassen, in der Summe jedoch erzielt die im Vergleich zur primären $360^{\circ}$ Fusion weniger aufwendige und für den Patienten weniger belastende dynamische Verschraubungsmethode in diesen (fusionierten) Patienten das gleiche Ergebnis. Diese Beobachtung wurde in der aktuellen Literatur bezüglich transpedikulärer dynamischer Systeme bislang nicht berichtet, wobei die meisten Untersuchungen sich auf eine Implantatkombination aus Titanschrauben und einer Polyesterkordel in 
Zusammenfassung

einem hohlen Platz- und Abstandhalter zwischen den Schrauben (Dynesys, Fa. Zimmer Spine) beziehen. Hier kann ein Zusammenhang zum verwendeten Implantatsystem in Form von Titanschrauben und einem Titanlängsträger angenommen werden.

Die Übertragbarkeit auf ähnliche Implantatsysteme sowie der Vergleich zu neuen dynamischen transpedikulären Verschraubungssystemen zum Beispiel mit Längsträgern aus PEEK und somit wiederum verändertem Einfluss auf die Biomechanik muss im Weiteren untersucht werden. 


\section{Anhang}

\subsection{Fragebogen für die klinische Untersuchung}

\section{Klinische Untersuchung}

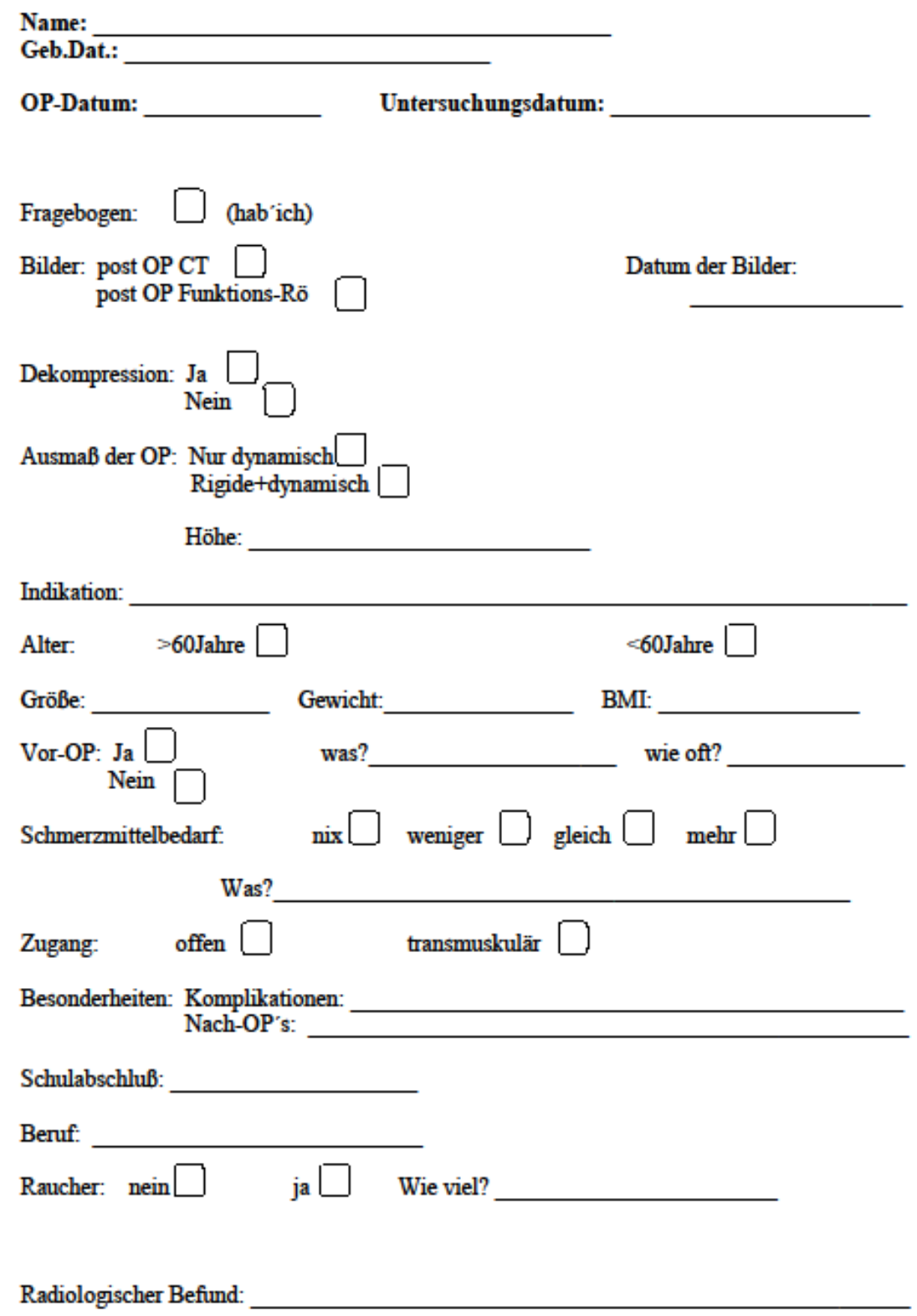




\subsection{Oswestry Disability Index}

\section{Patientenfragebogen}

(Bitte nur 1 Antwort (die für Sie am ehesten zutreffende Antwort) ankreuzen! Danke!)

Name, Vorname:

Geb.-Datum:

Frage 1: Schmerzintensität

0 : Ich habe momentan keine Schmerzen.

- 1: Ich habe momentan sehr leichte Schmerzen.

- 2: Ich habe momentan mäBige Schmerzen.

3: Ich habe momentan ziemlich starke Schmerzen.

- 4: Ich habe momentan sehr starke Schmerzen.

- 5: Die Schmerzen sind momentan die am schlimmsten vorstellbaren Schmerzen.

Frage 2: Persönliche Pflege (Körperpflege, Kleiden)

- 0: Ich kann mich selbst versorgen ohne zusätzliche Schmerzen auszulösen.

- 1: Ich kann mich selbst versorgen. Dies verursacht jedoch zusätzliche Schmerzen.

- 2: Es ist schmerzhaft mich selbst zu versorgen. Daher mache ich dies langsam und vorsichtig.

- 3: Ich benōtige Hilfe, jedoch kann ich den gröBten Teil meiner persönlichen Pflege selbst übermehmen.

- 4: Ich benōtige jeden Tag in den meisten Punkten meiner persönlichen Pflege Hilfe.

- 5: Ich schaffe es nicht, mich zu kleiden, waschen gelingt nur mit Mühe, ich bleibe im Bett.

Frage 3: Heben von Gegenständen

- 0: Ich kann schwere Gewichte ohne zusätzliche Schmerzen heben.

- 1: Ich kann schwere Gewichte heben. Dies verursacht jedoch zusätzliche Schmerzen.

- 2: Schmerzen hindern mich daran schwere Gewichte vom Boden zu heben. Jedoch kann ich diese heben, wenn sie in einer günstigen Position für mich liegen ( $\mathrm{z}$ B. auf dem Tisch)

- 3: Schmerzen hindern mich daran schwere Gewichte zu heben. Jedoch kann ich leichte bis mittelschwere Gewichte heben, wenn diese günstig für mich liegen.

- 4: Ich kann sehr leichte Gewichte heben

- 5: Ich kann weder etwas heben noch tragen.

\section{Frage 4: Laufen/Gehen}

0 : Schmerzen behindern mich nicht beim Gehen.

- 1: Schmerzen hindern mich daran weiter als $1000 \mathrm{~m}$ (am Stück) zu gehen.

2: Schmerzen hindern mich daran weiter als $500 \mathrm{~m}$ (am Stück) zu gehen.

- 3: Schmerzen hindern mich daran weiter als $100 \mathrm{~m}$ (am Stück) zu gehen.

4: Ich kann nur mit Hilfe eines Stockes oder mit Gehstützen gehen

- 5: Ich liege die meiste Zeit im Bett.

\section{Frage 5: Sitzen}

0 0: Ich kann auf jeder Art eines Stuhles sitzen so lange ich mōchte.

- 1: Ich kann auf meinem ,Lieblingsstuhl" sitzen so lange ich mōchte.

- 2: Schmerzen hindern mich daran länger als 1 Stunde zu sitzen.

- 3: Schmerzen hindern mich daran länger als 30 Minuten zu sitzen.

4: Schmerzen hindern mich daran länger als 10 Minuten zu sitzen.

- 5. Schmerzen halten mich vom Sitzen ab. 


\section{Frage 6: Stehen}

- 0: Ich kann ohne zusätzliche Schmerzen so lange stehen wie ich möchte

- 1: Ich kann so lange stehen wie ich möchte. Dies verursacht jedoch zusätzliche Schmerzen.

- 2: Schmerzen hindern mich daran länger als 1 Stunde zu stehen.

- 3: Schmerzen hindern mich daran länger als 30 Minuten zu stehen.

- 4: Schmerzen hindern mich daran länger als 10 Minuten zu stehen.

- 5: Schmerzen hindern mich grundsätzlich am Stehen.

\section{Frage 7: Schlaf:}

- 0: Mein Schlaf ist nie durch Schmerzen gestört.

- 1: Mein Schlaf ist gelegentlich durch Schmerzen gestört.

2: Auf Grund von Schmerzen schlafe ich weniger als 6 Stunden pro Nacht.

3: Auf Grund von Schmerzen schlafe ich weniger als 4 Stunden pro Nacht.

- 4: Auf Grund von Schmerzen schlafe ich weniger als 2 Stunden pro Nacht.

- 5: Schmerzen hindern mich grundsätzlich zu schlafen.

\section{Frage 8: Sexualleben:}

- 0: Mein Sexualleben ist uneingeschränkt und verursacht keine zusätzlichen Schmerzen

- 1: Mein Sexualleben ist uneingeschränkt, jedoch verursacht es zusätzliche Schmerzen

- 2: Mein Sexualleben ist annähernd normal, jedoch ist es sehr schmerzhaft.

3: Mein Sexualleben ist auf Grund der Schmerzen ziemlich eingeschränkt.

4: Mein Sexualleben ist auf Grund der Schmerzen äußerst eingeschränkt.

- 5: Schmerzen verhindern ein Sexualleben.

\section{Frage 9: Gesellschaftliches Leben}

- 0: Mein gesellschaftliches Leben ist uneingeschränkt und verursacht keine zusätzlichen Schmerzen.

- 1: Mein gesellschaftliches Leben ist uneingeschränkt, verursacht jedoch zusätzlichen Schmerzen.

- 2: Schmerzen haben keinen signifikanten Einfluss auf mein gesellschaftliches Leben, abgesehen von einer Einschränkung anstrengender Tätigkeiten (z.B. Sport).

- 3: Schmerzen schränken mein gesellschaftliches Leben ein und ich gehe nicht oft aus.

- 4: Schmerzen schränken mein gesellschaftliches Leben derart ein, dass ich fast nur noch zu Hause bleibe.

- 5: Auf Grund der Schmerzen habe ich überhaupt kein gesellschaftliches Leben mehr.

\section{Frage 10: Reisen}

- 0: Reisen verursacht keine zusätzlichen Schmerzen

- 1: Reisen verursacht zusätzliche Schmerzen.

- 2: Ich habe Schmerzen, jedoch kann ich Reisen mit einer Dauer über 2 Stunden unternehmen.

- 3: Auf Grund von Schmerzen beschränken sich meine Reisen auf eine Dauer von weniger als 1 Stunde.

- 4: Auf Grund von Schmerzen kann ich nur kurze, notwendige Reisen von weniger als 30 Minuten unternehmen.

- 5: Außer zu Behandlungen kann ich keine weiteren Reisen unternehmen. 


\subsection{Modifizierter Pationnaire-Patienten-Fragebogen}

\section{Patientenfragebogen NCH Gelnhausen (NCHGN01_LWS)}

Name, Vorname:

Datum:

Geb.-Datum: Wochen postoperativ Monate postoperativ Jahre postoperativ

\section{A. Meine Beinschmerzen VOR der Operation

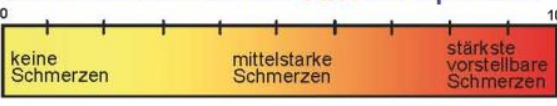 \\ Geben Sie mit Hilfe eines Kreuzes * die empfundenen Schmerzen an \\ 1.B. Meine Beinschmerzen NACH der Operation

\begin{tabular}{|l|l|l|}
\hline $\begin{array}{l}\text { mittelstarke } \\
\text { Sche } \\
\text { Schmerzen }\end{array}$ & $\begin{array}{l}\text { stärkste } \\
\text { vorstellbare }\end{array}$ \\
\hline Schmerzen
\end{tabular} \\ Geben Sie mit Hilfe eines Kreuzes $x$ die empfundenen Schmerzen an}

3. Meine Beschwerden vor der Operation sind im Vergleich ...

A. Schmerzen bei Belastung/ Bewegung

$\square$ hatte ich nich

B. Schmerzen in Ruhe

hatte ich nich

C. Schmerzen in der Nacht

口 hatte ich nicht

D. Brennen

hatte ich nicht

E. Krämpfe

$\square$ hatte ich nicht

F. Kraftlosigkeit/Schwäche

$\square$ hatte ich nicht

G. Gefühlsstörungen, Kribbeln, Ameisenlaufen

口 hatte ich nicht

H. Steifheit

$\square$ hatte ich nich vie

I. Bewegungseinschränkung

a hatte ich nicht viel besser besser gleich schlechter viel schlechter

viel besser besser gleich schlechter viel schlechter

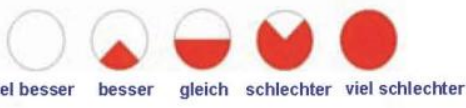
viel besser besser gleich schlechter viel schlechter

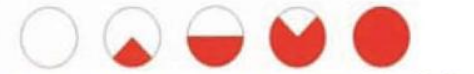
viel besser besser gleich schlechter viel schlechter

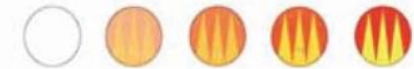

viel besser besser gleich schlechter viel schlechter
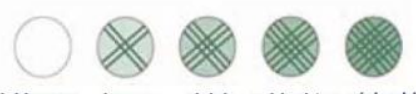

gleich schlechter viel schlecter
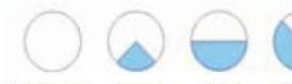

viel besser besser gleich schlechter viel schlechter

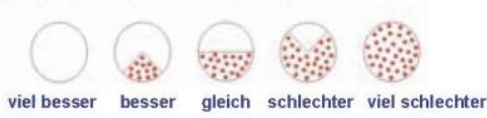

viel besser besser gleich schlechter viel schlechter
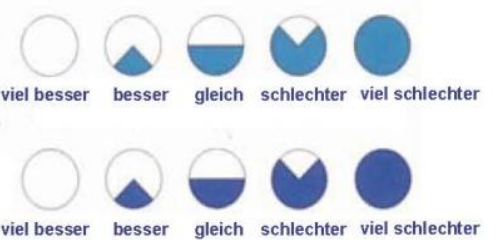

2.A. Meine Rückenschmerzen VOR der Operation

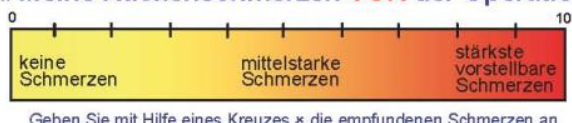

2.B. Meine Rückenschmerzen NACH der Operation

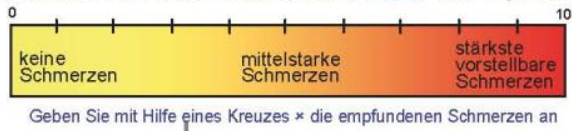

Bitte Ort der Beschwerden mit $\times$ einzeichnen...
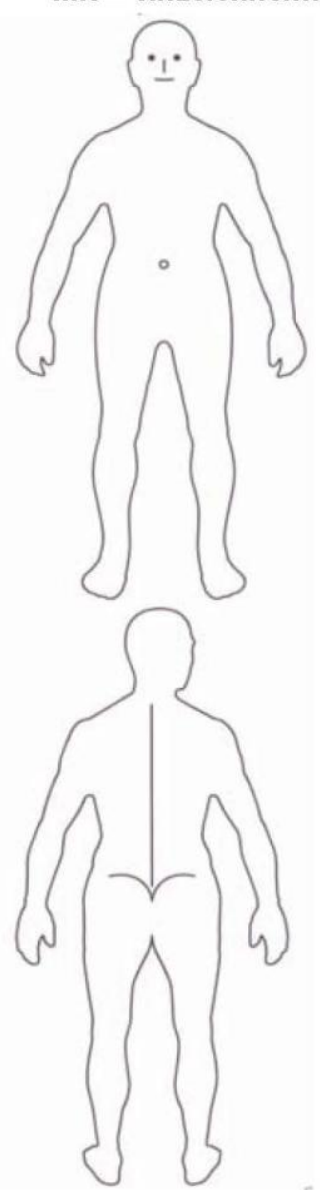
4. Meine Einschränkungen sind im Vergleich zu vor der Operation ...
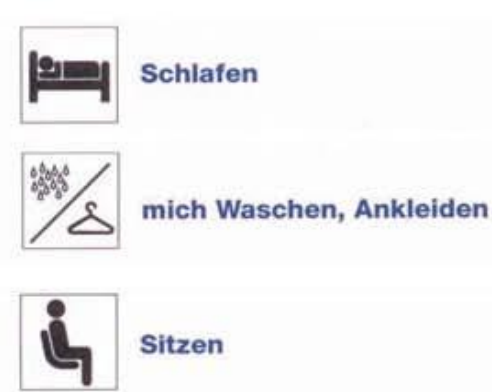

에 Stehen

1․ Gehen

Wip
Ausgehen, Freundebesuchen (soziales Leben)

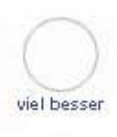

$\bigcup_{\text {viel besser }}$ vesser

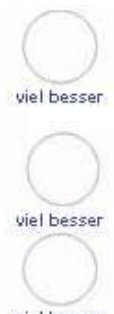

viel besser

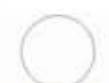

viel besser
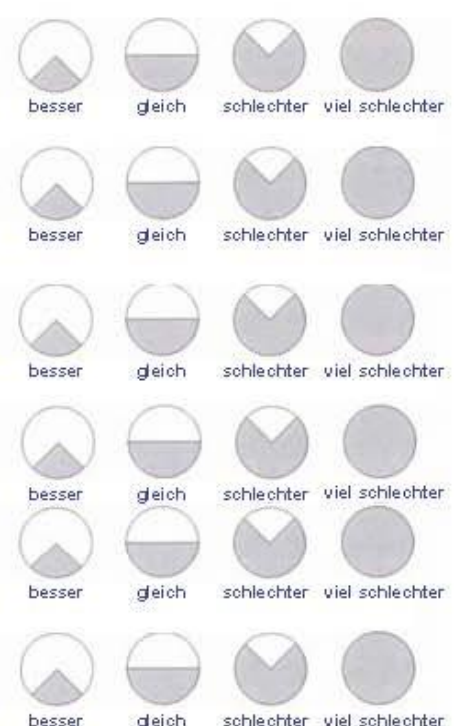

5. Sind Sie seit der OP in einer anderen Klinik nochmals an der Wirbelsäule operiert worden? $\square$ nein $\square$ ja.

5.A. Wenn ja, wann: wo: warum:

6. Wie zufrieden sind Sie mit Ihrer Behandlung?

6.A. In der Praxis?

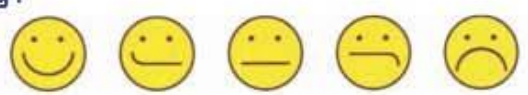

6.B. Im Krankenhaus?

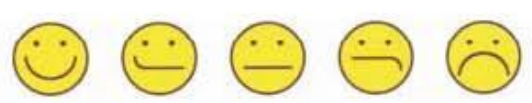

6.C. Insgesamt?

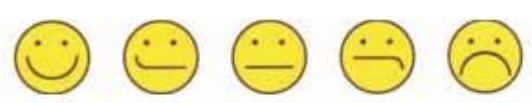

7. Wie zufrieden sind Sie mit Ihrer jetzigen Situation?

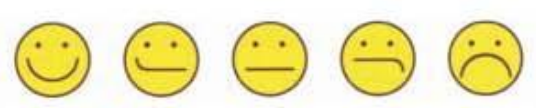

8. Würden Sie sich nochmal operieren lassen? $\square$ ja $\square$ nein.

8.A. Wenn nein, warum nicht:

9. Kommentare, Wünsche, Anregungen, Kritik 


\subsection{Abbildungsverzeichnis}

Abbildung 1: Umfang der Operation........................................ 11

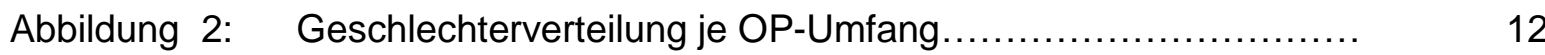

Abbildung 3: $\quad$ Anzahl der betroffenen Segmente.............................. 12

Abbildung 4: Höhenverteilung aller Dynamischen Systeme inklusive

Topping off................................................ 14

Abbildung 5: Höhenverteilung aller rigiden Fusionen mit Topping off im

Anschlusssegment......................................... 15

Abbildung 6: Quote der dekomprimierten Spinalkanalstenosen............... 15

Abbildung 7: Anschlussinstabilität im Funktionsröntgen..................... 20

Abbildung 8: Anschlussfraktur........................................ $\quad 20$

Abbildung 9: Schraubenlockerung links.................................... 21

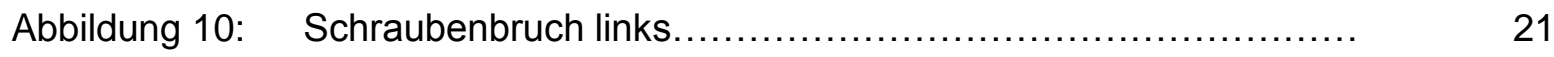

Abbildung 11: $\quad$ Gelenkfusion rechts........................................ 22

Abbildung 12: Rigide (hinten) und dynamische (vorne) Schrauben................ 23

Abbildung 13: $\quad$ Platten des Bricon DSS ...................................... 23

Abbildung 14: Dynamisches Implantat........................................ 23

Abbildung 15: Häufigkeit Voroperationen............................... 28

Abbildung 16: Vergleich postoperativer Schmerzmittelbedarf zu

präoperativem Schmerzmittelbedarf........................ 29 
Abbildung 17: Vergleich postoperativer Schmerzmittelbedarf nach

Operationsumfang.

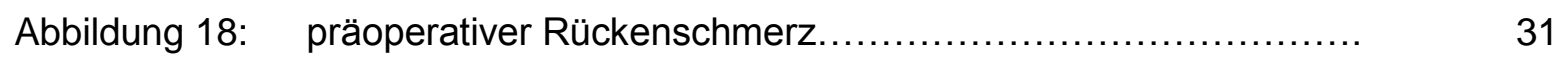

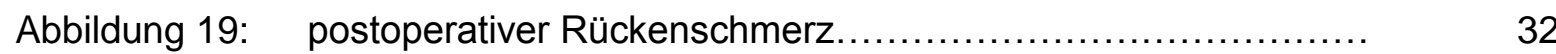

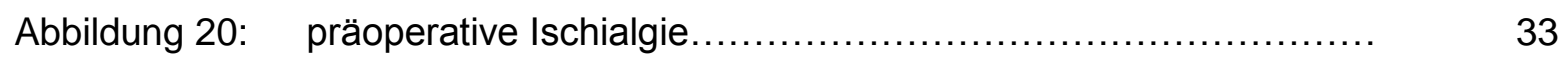

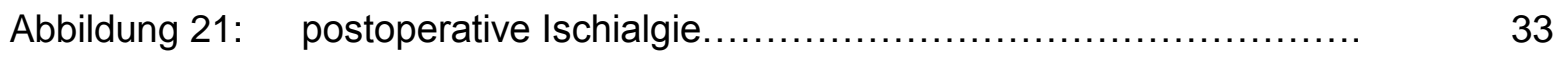

Abbildung 22: Schmerzen bei Belastung postoperativ im Vergleich zu

präoperativ

Abbildung 23: Schmerzen in Ruhe postoperativ im Vergleich zu

präoperativ

Abbildung 24: Schmerzen nachts postoperativ im Vergleich zu präoperativ....

Abbildung 25: Brenndysästhesien postoperativ im Vergleich zu präoperativ....

Abbildung 26: Krämpfe postoperativ im Vergleich zu präoperativ

Abbildung 27: Schwäche / Kraftlosigkeit postoperativ im Vergleich zu

präoperativ

Abbildung 28: $\quad$ Parästhesien postoperativ im Vergleich zu präoperativ............ 40

Abbildung 29: Steifheit postoperativ im Vergleich zu präoperativ.................

Abbildung 30: Bewegungseinschränkung postoperativ im Vergleich zu

präoperativ.

Abbildung 31: Veränderung des Schlafs postoperativ im Vergleich zu

präoperativ. 
Abbildung 32: Veränderung beim Waschen / Kleiden postoperativ im Vergleich zu präoperativ.

Abbildung 33: Veränderung Sitzfähigkeit postoperativ im Vergleich zu

präoperativ

Abbildung 34: Veränderung Stehfähigkeit postoperativ im Vergleich zu

präoperativ

Abbildung 35: Veränderung Gehfähigkeit postoperativ im Vergleich zu präoperativ

Abbildung 36: Veränderung der Teilnahme am Sozialen Leben

postoperativ im Vergleich zu präoperativ.

Abbildung 37: Zufriedenheit mit postoperativer Situation.

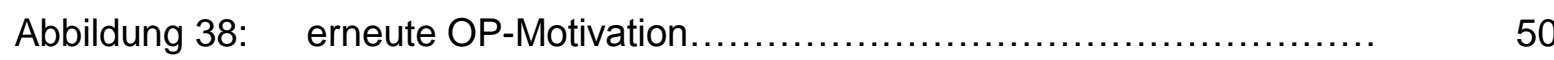

Abbildung 39: Verteilung Oswestry-Score in Bezug auf OP-Umfang.............. 51

Abbildung 40: Häufigkeitsverteilung der Beeinträchtigung Oswestry-Score.....

Abbildung 41: Häufigkeitsverteilung Höhe der Schraubenlockerung............. 52

Abbildung 42: $\quad$ Fusionsrate bezogen auf OP-Umfang ........................... 53

Abbildung 43: postoperativer Rückenschmerz bezogen auf Fusion............. 54

Abbildung 44: Häufigkeitsverteilung Anschlussinstabilität bezogen auf

OP-Umfang

Abbildung 45: Häufigkeitsverteilung Fusion bezogen auf Alter 
Anhang

Abbildung 46: postoperativer Schmerzmittelbedarf im Vergleich zu

präoperativ unter Berücksichtigung Anschlussinstabilität.........

Abbildung 47: Häufigkeitsverteilung Fusion bezogen auf Schrauben-

Lockerung ...

62

Abbildung 48: Fusion bezogen auf $\mathrm{BMl}$ in $\mathrm{kg} / \mathrm{m}^{2}$ 


\subsection{Tabellenverzeichnis}

Tabelle 1:

Höhenverteilung Dynamische Fusion

13

Tabelle 2:

Höhenverteilung Dynamisch plus rigide Fusion

14

Tabelle 3:

Body-Mass-Index

27

Tabelle 4:

Auswertung Oswestry-Score

50

Tabelle 5:

Häufigkeitsverteilung Höhe der Schraubenbrüche

53

Tabelle 6:

Einfluss des Rauchens auf die Fusionsrate

56

Tabelle 7:

Chi²-Test: Einfluss Rauchen auf Fusion

56

Tabelle 8:

Einfluss des Alters auf die Fusionsrate

57

Tabelle 9:

Chi²-Test: Einfluss Alter auf Fusion

57

Tabelle 10:

Tabelle 10: Einfluss des OP-Umfangs auf das

Auftreten einer Anschlussinstabilität

Tabelle 11:

Chi'2-Test: Einfluss OP-Umfang auf

59

Anschlussinstabilität

Tabelle 12

Einfluss Rauchen auf Schraubenlockerung

60

Tabelle 13:

Chi²-Test: Einfluss Rauchen auf Schraubenlockerung

60

Tabelle 14:

P-Werte, Mann-Whitney-U-Test

61

Tabelle 15:

Mann-Whitney-U-Test: Einfluss Schraubenlockerung

62 auf Fusion

Tabelle 16:

Mann-Whitney-U-Test: Einfluss Dekompression auf postoperative Ischialgie

Tabelle 17:

P-Werte logistische Regression 


\section{Literaturverzeichnis}

Adams MA, Hutton WC (1983): The mechanical function of the lumbar apophyseal joints. Spine $\underline{8}, 327-330$

Bambakidis NC, Feiz-Erfan I, Klopfenstein JD, Sonntag VK (2005): Indications for surgical fusion of the cervical and lumbar motion segment. Spine $\underline{30}$, S2-S6

Baur-Melnyk A, Triantafyllou M, Birkenmaier C, Reiser M (2006): Degenerative Erkrankungen der Wirbelsäule: Seltene und oft verkannte Ursachen von Schmerzsyndromen. Radiologe $\underline{46}$, 454-476

Boos N, Webb JK (1997): Pedicle screw fixation in spinal disorders: a European view. Eur Spine J $\underline{6}, 2-18$

Bothmann M, Kast E, Boldt GJ, Oberle J (2008): Dynesys fixation for lumbar spine degeneration. Neurosurg Rev $\underline{31}, 189-196$

Bridwell KH, Sedgewick TA, O'Brien MF, Lenke LG, Baldus C (1993): The role of fusion and instrumentation in the treatment of degenerative spondylolisthesis with spinal stenosis. J Spinal Disord $\underline{6}$, 461-472

Cakir B, Ulmar B, Koepp H, Huch K, Puhl W, Richter M (2003): Posterior dynamic stabilization as an alternative for instrumented fusion in the treatment of degenerative lumbar instability with spinal stenosis. Z Orthop Ihre Grenzgeb 141, 418-424

Cakir B, Carazzo C, Schmidt R, Mattes T, Reichel H, Käfer W (2009): Adjacent Segment Mobility After Rigid and Semirigid Instrumentation of the Lumbar Spine. Spine 34, 1287-1291

Carragee EJ, Alamin TF, Miller JL, Caragee JM (2005): Discographic, MRI and psychosocial determinants of low back pain disability and remission: a prospective Study in subjects with benign persistent back pain. Spine J $\underline{5}, 24-35$ 
Cesar-Neto JB, Duarte PM, Sallum EA, Barbieri D, Moreno H Jr, Nociti FH Jr (2003): A comperative study on the effect of nicotine administration and cigarette smoke inhalation on bone healing around titanium implants. J Peridontol $\underline{74}$ (10): 1454-1459 Cheng BC, Gordon J, Cheng J, Welch W (2007): Immediate Biomechanical Effects of Lumbar Posterior Dynamic Stabilization Above a Circumferential Fusion. Spine $\underline{32}$, 2551-2557

Chou WY, Hsu CJ, Chang WN, Wong CY (2002): Adjacent segment degeneration after lumbar spinal posterolateral fusion with instrumentation in elderly patients. Arch Orthop Trauma Surg 122, 39-43

Davidson M, Keating J (2002): A Comparison of Five Low Back Disability Questionnaires: Reliability and Responsiveness. Phys Ther 82, 8-24

Deyo RA, Ciol MA, Cherkin DC, Loeser JD, Bigos SJ (1993): Lumbar Spinal Fusion: A Cohort Study of Complications, Reoperations and Resource Use in the Medicare Population. Spine 18, 1463-1470

DiPierro CG, Helm GA, Shaffrey CI, Chadduck JB, Henson SL, Malik JM, Szabo TA, Simmons NE, Jane JA (1996): Treatment of lumbar spinal stenosis by extensive unilateral decompression and contralateral autologous bone fusion: operative technique and results. J Neurosurg $\underline{84}, 166-173$

Etebar S, Cahill DW (1999): Risk factors for adjacent-segment failure following lumbar fixation with rigid instrumentation for degenerative instability. J Neurosurg $\underline{90}$ (2Suppl), 163-169

Fairbank JCT (2007): Use and Abuse of Oswestry Disability Index. Spine 32, 27872789

Fairbank JC, Pynsent PB (2000): The Oswestry Disability Index. Spine 25, 29402952

Fairbank JC, Couper J, O’Brien JP (1980): The Oswestry low back pain questionnaire. Physiotherapy $\underline{66}, 271-273$ 
Fernández-Fairen M, Sala P, Ramírez H, Gil J (2007): A Prospective Randomized Study of Unilateral VersusBilateral Instrumented Postolateral Lumbar Fusion in Degenerative Spondylolisthesis. Spine 32, 395-401

Fischgrund JS, Mackay M, Herkowitz HN, Brower R, Montgomery DM, Kurz LT (1997): Volvo Award winner in clinical studies. Degenerative lumbar spondylolisthesis with spinal stenosis: A prospective, randomized study comparing decompressive laminectomy and arthrodesis with and without spinal instrumentation. Spine $\underline{22}$, 2807-2012

Fritzell P, Hägg O, Jonsson D, Nordwall A (2004): Cost-effectiveness of lumbar fusion and nonsurgical treatment for chronic low back pain in the Swedisch Lumbaar Spine Study: a multicenter, randomized, controlled trial from the Swedish Lumbar Spine Study Group. Spine 29, 421-434

Gédet P, Haschtmann D, Thistlethwaite PA, Ferguson SJ (2009): Comparative biomechanical investigation of a modular dynamic lumbar stabilization system and the Dynesys system. Eur Spine J $\underline{18}, 1504-1511$

Gibson JN, Waddell G (2005): Surgery for degenerative lumbar spondylosis: updated Cochrane review. Spine $\underline{30}, 2312-2320$

Gibson JN, Grant JC, Waddell G (1999): The Cochrane review of surgery for lumbar disc prolapse and degenerative lumbar spondylosis. Spine $\underline{24}, 1820-1832$

Glaser J, Stanley M, Sayre H, Wooda J, Found E, Spratt K (2003): A 10-year followup evaluation of lumbar spine fusion with pedicle screw fixation. Spine $\underline{28}, 1390-1395$

Grevitt MP, Gardner ADH, Spilsbury J, Shackleford IM, Baskerville R, Pursell LM, Hassaan A, Mulholland RC (1995): The Graf stabilisation system: early results in 50 patients. Eur Spine J $\underline{4}, 169-175$

Grob D, Benini A, Junge A, Mannion AF (2005): Clinical experience with the Dynesys semirigid fixation system for the lumbar spine: surgical and patient-oriented outcome in 50 cases after an average of 2 years. Spine $\underline{30}, 324-331$ 
Grob D, Humke T, Dvorak J (1995): Degenerative lumbar spinal stenosis.

Decompression with and without arthrodesis. J Bone Joint Surg Am. $\underline{77}, 1036-1041$

Haberl H, Cripton PA, Orr TE, Beutler T, Frei H, Lanksch WR, Nolte LP (2004):

Kinematic response of lumbar functional spinal units to axial torsion with and without superimposed compression and flexion/extension. Eur Spine J $\underline{13}$, 560-566

Hazard RG, Bendix A, Fenwick JW (1991): Disability exaggeration as apredictor of functional restoration outcomes for patients with chronic low-back pain. Spine $\underline{16}$, 1062-1067

Herkowitz HN, Kurz LT (1991): Degenerative lumbar spondylolisthesis with spinal stenosis: a prospective study comparing decompression with decompression and intertransverse process arthrodesis. J Bone Joint Surg $\mathrm{Br} \underline{73}, 802-808$

Herno A, Airaksinen O, Saari T (1994): Computed tomography after laminectomy for lumbar pinal stenosis. Patients' pain paterns, walking capacity, and subjective disability had no correlation with computed tomography findings. Spine $\underline{19}, 1975-$ 1978

Highsmith JM, Tumialán LM, Rodts Jr GE (2007): Flexible rods and the case for dynamic stabilization. Neurosurg Focus $\underline{22}$ (1): E1

Johnston CE II, Ashman RB, Baird AM, Allard RN (1990): Effect of spinal construct stiffness on early fusion mass incorporation: experimental study. Spine $\underline{15}, 908-912$ Jönsson B, Annertz M, Sjöberg C, Strömqvist B (1997): A prospective, consecutive study of surgically treated lumbar spinal stenosis. Part II: Five-year follow-up by an independant observer. Spine 22, 2938-2944

Katz JN, Stucki G, Lipson SJ, Fossel AH, Grobler LJ, Weinstein JN (1999):

Predictors of surgical outcome in degenerative lumbar spinal stenosis. Spine $\underline{24}$, 2229-2233

Khoueir P, Kim A, Wang MY (2007): Classification of posterior dynamic stabilization devices. Neurosurg Focus $\underline{22}$ (1): E3 
Kim DH, Cammisa Jr. FP, Fessler RG: Dynamic Reconstruction of the Spine. Thieme Medical Pulishers, Inc., New York 2006

Kim YS, Zhang HY, Moon BJ, Park KW, Ji KY, Lee WC, Oh KS, Ryu GU, Kim DH (2007): Nitinol spring rod dynamic stabilization system and Nitinol memory loops in surgical treatment for lumbar disc disorders: short-term follow up. Neurosurg Focus $\underline{22}(1): E 10$

Kirkaldy-Willis WH, Farfan HF (1982): Instability of the Lumbar Spine. Clinical Orthopaedics and Related Research $\underline{165}, 110-123$

Korovessis P, Papazisis Z, Koureas G, Lambiris E (2004): Rigid, Semirigid Versus dynamic Instrumentation for Degenerative Lumbar Spinal Stenosis: A Correlative Radiological and Clinical Analysis of Short-Term Results. Spine 29, 735-742 Krismer M, Haid C, Behensky H, Kapfinger P, Landauer F, Rachbauer F (2000): Motion in Lumbar Functional Spine Units During Side Bending and Axial Rotation Moments Depending on the Degree of Degeneration. Spine 25, 2020-2027

Kumar MN, Baklanov A, Chopin D (2001): Correlation between sagittal plane changes and adjacent segment degeneration following lumbar spine fusion. Eur Spine J 10, 314-319

Lafage V, Gangnet N, Sénégas J, Lavaste F, Skalli W (2007): New Interspinous Implantat Evaluation Using an In Vitro Biomechanical Study Combined With a Finite Element Analysis. Spine 32, 1706-1713

Lawthorne III TW, Girardi FP, Mina CA Pappou I, Cammisa Jr FP (2009): Treatment of degenerative spondylolisthesis: potential impact of dynamic stabilization based on imaging analysis. Eur Spine J $\underline{18}, 815-822$

Lee CK (1988): Accelerated degeneration of the segment adjacent to a lumbar fusion. Spine $\underline{13}, 375-377$ 
Lee SE, Park SB, Jahng TA, Chung CK, Kim HJ (2008): Clinical Experience of the Dynamic Stabilization System for the Degenerative Spine Disease. J Korean Neurosurg Soc $\underline{43}, 221-226$

Lonstein JE, Denis F, Perra JH, Pinto MR, Smith MD, Winter RB (1999):

Complications associated with pedicle screws. J Bone Joint Surg Am $\underline{81}$, 1519-1528

Lotz JC, Ulrich JA (2006): Innervation, inflammation, and hypermobility may characterize pathologic disc degenerstion: review of animal model data. J Bone Joint Surg Am $\underline{88}$ (suppl2), 76-82

Mandigo CE, Sampath P, Kaiser M (2007): Posterior dynamic stabilization of the lumbar spine: pedicle-based stabilization with the AccuFlex rod system. Neurosurg Focus $\underline{22}(1)$ : E9

Mardjetko SM, Connolly PJ, Shott S (1994): Degenerative lumbar spondylolisthesis: a meta-analysis of literature 1970-1993. Spine 19 (20 Suppl), 2256-2265

Markwalder TM, Wenger M (2002): Adjacent-segment morbidity. J Neurosurg $\underline{96}$ (2Suppl), 139-140

Martin CR, Gruszczynski AT, Braunsfurth HA, Fallatah SM, O’Neil J, Wai EK (2007): The Surgical Management of Degenerative Lumbar Spondylolisthesis. Spine $\underline{32}$, 1791-1798

Möller H, Hedlund R (2000): Surgery versus Conservative Management in Adult Isthmic Spondylolisthesis. Spine $\underline{25}, 1711-1715$

Mohan A, Das K (2003): History of surgery for the correction of spinal deformity. Neurosurg Focus 14 (1): E1

Mummaneni PV, Haid RW, Rodts GE (2004): Lumbar interbody fusion: state-of-theart technical advances. J Neurosurg Spine 1 (1), 24-30 
Niosi CA, Zhu Q, Wilson DC, Keynan O, Wilson DR, Oxland TR (2006):

Biomechanical characterization of the three- dimensional kinematic behaviour of the Dynesys dynamic stabilization system: an in vitro study. Eur Spine J 15, 913-922

Niosi CA, Wilson DC, Zhu Q, Keynan O, Wilson DR, Oxland TR (2008): The Effect of Dynamic Posterior Stabilization on Facet Joint Contact Forces : An In Vitro Investigation. Spine $\underline{33}, 19-26$

Ostelo RWJG, Deyo RA, Stratford P, Waddell G, Croft P, Von Korff M, Bouter LM, de Vet HC (2008): Interpreting Change Scores for Pain and Functional Status in Low Back Pain. Spine $\underline{33}, 90-94$

Pellisé F, Vidal X, Hernández A, Cedraschi C, Bagó J, Villanueva C (2005):

Reliability of Retrospective Clinical Data to Evaluate the Effectiveness of Lumbar Fusion in Chronic Low Back Pain. Spine $\underline{30}$, 365-368

Pellisé F, Hernández A, Vidal X, Minguell J, Martínez C, Villanueva C (2007):

Radiologic assessment of all unfused lumbar segments 7.5 years after instrumented posterior spinal fusion. Spine $\underline{32}, 574-579$

Postacchini F, Cinotti G (1992): Bone regrowth after surgical decompression for lumbar spinal stenosis. J Bone Joint Surg $\mathrm{Br} \underline{74}, 862-869$

Pratt RK, Fairbank JC, Virr A (2002): The reliability of the Shuttle Walking Test, the Swiss Spinal Stenosis Questionnaire, the Oxford Spinal Stenosis Score, and the Oswestry Disability Index in the assessment of patients with lumbar spinal stenosis. Spine 27, 84-91

Quint U, Wilke HJ, Löer F, Claes L (1998): Statische und dynamische Stabilisierungsmöglichkeiten der Wirbelsäule bei Läsionen des anterioren und posterioren Ligamentkomplexes. Unfallchirurg 101, 684-690

Rahm MD, Hall BB (1996): Adjacent-segment degeneration after lumbar fusion with instrumentation: a retrospective study. J Spinal Disord $\underline{9}$, 392-400 
Rechtine GR, Sutterlin CE, Wood GW, Boyd RJ, Mansfield FL (1996): The efficacy of pedicle screw/plate fixation on lumbar/lumbosacral autogenous bone graft fusion in adult patients with degenerative spondylolisthesis. J Spinal Disord $\underline{9}, 382-391$

Resnick DK, Choudhri TF, Dailey AT, Groff MW, Khoo L, Matz PG, Mummaneni P, Watters WC 3rd, Wang J, Walters BC (2005): Guidelines for the performance of fusion procedures for degenerative disease of the lumbar spine. Part 5: correlation between radiographic and functional outcome. J Neurosurg Spine $\underline{2}$, 658-661

Schaeren S, Broger I, Jeanneret B (2008): Minimum Four-Year Follow-up of Spinal Stenosis with Degenerative Spondylolisthesis Treated With Decompression and Dynamic Stabilization. Spine 33, E636-E642

Schmidt H, Heuer F, Wilke HJ (2008): Which axial and bending stiffness of posterior implants are required to design a flexible lumbar stabilization system? J Biomech, 1-7

Schmoelz W, Huber JF, Nydegger T, Claes L, Wilke HJ (2006): Influence of a dynamic stabilisation system on load bearing of a bridged disc: an in vitro study of intradiscal pressure. Eur Spine J $\underline{15}, 1276-1285$

Schmoelz W, Onder U, Martin A, von Strempel A (2009): Non-fusion instrumentation of the lumbar spine with a hinged pedicle screw rod system: an in vitro experiment. Eur Spine J $\underline{18}, 1478-1485$

Schnake KJ, Schaeren S, Jeanneret B (2006): Dynamic Stabilization in Addition to Decompression for Lumbar Spinal Stenosis with Degenerative Spondylolisthesis. Spine $\underline{31}, 442-449$

Schulte TL, Bullmann V, Lerner T, Schneider M, Marquardt B, Liljenqvist U, Pietilä TA, Hackenberg L (2006): Lumbale Spinalkanalstenose. Orthopäde $\underline{35}, 675-694$

Schulte TL, Hurschler C, Haversath M, Liljenqvist U, Bullmann V, Filler TJ, Osada N, Fallenberg EM, Hackenberg $L$ (2008): The effect of dynamic, semi-rigid implants on the range of motion of lumbar motion segments after decompression. Eur Spine $\mathrm{J} \underline{17}$, 1057-1065 
Sengupta DK, Herkowitz HN (2005): Degenerative spondylolisthesis: review of current trends and controversies. Spine $\underline{30}$, S71-S81

Sengupta DK, Mulholland RC (2005): Fulcrum assisted soft stabilization system: a new concept in the surgical treatment of degenerative low back pain. Spine $\underline{30}, 1019$ 1030

Serhan HA, Varnavas G, Dooris AP, Patwardhan A, Tzermiadianos M (2007):

Biomechanics of the posterior lumbar articulating elements. Neurosurg Focus $\underline{22}$ (1): E1

Sirvanci M, Bhatia M, Ganiyusufoglu KA, Duran C, Tezer M, Ozturk C, Aydogan M, Hamzaoglu (2008): Degenerative lumbar spinal stenosis: correlation with Oswestry Disability Index and MR Imaging. Eur Spine J 17, 679-685

Toyone T, Tanaka T, Kato D, Kaneyama R, Otsuka M (2005): Patient` expectations and satisfaction in lumbar spine surgery. Spine $\underline{30}, 2689-2694$

Troum OM, Crues JV III (2004): The young adult with hip pain: diagnosis and medical treatment. Clin Orthop Relat Res $\underline{418}$, 9-17

Turner JA, Ersek M, Herron L, Deyo R (1992a): Surgery for lumbar spinal stenosis. Attemted meta-analysis of the lierature. Spine $\underline{17}, 1-8$

Turner JA, Ersek M, Herron L, Haselkorn J, Kent D, Ciol MA, Deyo R (1992b):

Patient outcomes after lumbar spinal fusions. JAMA 268, 907-911

Vaga S, Brayda-Bruno M, Perona F, Fornari M, Raimondi MT, Petruzzi M, Grava G, Costa F, Caiani EG, Lamartina C (2009): Molecular MR imaging for the evaluation of the effect of dynamic stabilization on lumbar intervertebral discs. Eur Spine $J$ $\underline{18}$,(Suppl 1), S40-S48

Weinstein JN, Tosteson TD, Lurie JD, Tosteson ANA, Blood E, Hanscom B, Herkowitz H, Cammisa F, Albert T, Boden SD (2008): Surgical versus Nonsurgical Therapy for Lumbar Spinal Stenosis. N Engl J Med 358, 794-810 
Welch WC, Cheng BC, Awad TE, Davis R, Maxwell JH, Delamarter R, Wingate JK, Sherman J, Macenski MM (2007): Clinical outcomes of the Dynesys dynamic neutralization system: 1-year preliminary results. Neurosurg Focus $\underline{22}$ (1): E8

Wilke HJ, Heuer F, Schmidt H (2009): Prospective Design Delineation and Subsequent InVitro Evaluation of a New Posterior Dynamic Stabilization System. Spine $\underline{34}$, 255-261

Würgler-Hauri CC, Kalbarczyk A, Wiesli M, Landolt H, Fandino J (2008): Dynamic Neutralization of the Lumbar Spine After Microsurgical Decompression in Aquired Lumbar Spinal Stenosis and Segmental Instability. Spine 33, E66-E72

Yamamoto I, Panjabi MM, Crisco T, Oxland T (1989): Three-dimensional movements of the whole lumbar spine and lumbosacral joint. Spine $\underline{14}, 1256-1260$

Yang KH, King Al (1984): Mechanism of facet load transmission as a hypothesis for low-back pain. Spine $\underline{9}, 557-565$

Yaun HA, Garfin SR, Dickmann CA, Mardjetko SM (1994): A historical cohort study of pedicle screw fixation in thoracic, lumbar, and sacral spinal fusions. Spine $\underline{19}$ (20Suppl),2279-2296

Yee A, Adjei N, Do J, Ford M, Finkelstein J (2008): Do Patient Expectations of Spinal Surgery Relate to Functional Outcome? Clin Orthop Relat Res $\underline{466}, 1154-1161$ Young PM, Berquist TH, Bancroft LW, Peterson JJ (2007): Complications of Spinal Instrumentation. RadioGraphics $\underline{27}, 775-789$ 


\section{Danksagung}

Mein vornehmlicher Dank gilt meinem Doktorvater, Herrn Prof. Dr. med. V. Rohde, der es mir ermöglichte, diese Arbeit in seiner Abteilung anfertigen zu können und mich mit seinem Interesse an der Fragestellung und dem Gesamtthema sehr motiviert hat.

Für die Unterstützung bei der Entwicklung des Themas der Arbeit, der Umsetzung in eine wissenschaftliche Fragestellung mit Erarbeitung der durchzuführenden Untersuchungen und die Begleitung zur Umsetzung ab der ersten Idee möchte ich mich ganz herzlich bei Dr. med. Bernd M. Hölper bedanken.

Mein ganz besonderer Dank gilt Dr. med. Michael K. Eichler, der mir nicht erst seit Beginn dieser Arbeit jederzeit mit Rat und Tat hilfreich zur Seite steht. Ebenso bedanke ich mich für das hohe Engagement und die vielen aufbauenden Gespräche während der Durchführung bis zum Abschluss der Dissertation.

Für die Unterstützung bei der statistischen Auswertung der Ergebnisse möchte ich der Abteilung Medizinische Statistik der Universität Göttingen, insbesondere Herrn Dr. Frank Konietschke, danken.

Dem kompletten Praxisteam meinen herzlichen Dank für die Hilfe bei der organisatorischen Durchführung der Datenerhebung, sowie für die vielen freundlichen und aufmunternden Worte. 


\section{$\underline{\text { Lebenslauf }}$}

Am 07.06.1981 wurde ich, Sabrina Monika Greve, als erstes Kind meiner Eltern Magdalena und Bernhard Greve in Fulda geboren.

Von 1987 bis 1991 besuchte ich die „Conrad-von-Mengersen-Schule“, Johannesberg und wechselte danach auf die „Heinrich-von-Bibra-Schule“, Fulda, die ich 1997 mit dem Abschluss der Mittleren Reife beendete.

Im Anschluss leistete ich ein einjähriges Vorpraktikum in der Nephrologischen Klinik des Klinikums Fulda ab. Hierauf folgte eine dreijährige Ausbildung zur Krankenschwester an der Krankenpflegeschule des Klinikums Fulda. Diese schloss ich im September 2001 mit dem Staatsexamen „Krankenpflege“ ab.

Von Oktober 2001 bis September 2004 arbeitete ich als examinierte Krankenschwester auf der Intensiv-Pflege-Einheit (IPE) der Neurochirurgischen Klinik des Klinikums Fulda.

Von 2003 bis 2004 besuchte ich den Vorbereitungskurs zum Erwerb der fachbezogenen Hochschulreife des „Stephanstifts“, Hannover. Die fachbezogene Hochschulreife erwarb ich im Juli 2004.

Zum Wintersemester 2004/2005 nahm ich das Studium der Humanmedizin an der Georg-August-Universität Göttingen auf. Im Sommer 2007 legte ich den 1. Abschnitt der ärztlichen Prüfung ab.

Im Herbst 2008 nahm ich die Arbeit an der vorliegenden Dissertation auf.

Parallel zum Studium arbeitete ich drei weitere Jahre als Teilzeitkraft in der Neurochirurgischen Klinik des Klinikums Fulda und anschließend ein Jahr in der Altenpflege im Alten- und Pflegeheim Marienheim in Fulda. Seit Sommer 2008 bin ich in der vorlesungsfreien Zeit als wissenschaftliche Assistentin der Neurochirurgie Osthessen GbR tätig. 